\title{
Yang-Mills fields on CR manifolds
}

\author{
Elisabetta Barletta Sorin Dragomir \\ Università degli Studi della Basilicata, \\ Dipartimento di Matematica, \\ Campus Macchia Romana, \\ 85100 Potenza, Italy, \\ e-mail: barletta@unibas.it dragomir@unibas.it \\ Hajime Urakawa \\ Division of Mathematics \\ Graduate School of Information Sciences \\ Tohoku University \\ Aoba 09, Sendai, 980-8579, Japan \\ e-mail: urakawa@math.is.tohoku.ac.jp
}

\begin{abstract}
We study pseudo Yang-Mills fields on a compact strictly pseudoconvex $\mathrm{CR}$ manifold $M$, i.e. the critical points of the functional $\mathcal{P} \mathcal{Y} \mathcal{M}(D)=\frac{1}{2} \int_{M}\left\|\pi_{H} R^{D}\right\|^{2} \theta \wedge(d \theta)^{n}$, where $D$ is a connection in a Hermitian CR-holomorphic vector bundle $(E, h) \rightarrow$ $M$. Let $\Omega=\{\varphi<0\} \subset \mathbb{C}^{n}$ be a smoothly bounded strictly pseuodoconvex domain and $g$ the Bergman metric on $\Omega$. We show that boundary values $D_{b}$ of Yang-Mills fields $D$ on $(\Omega, g)$ are pseudo Yang-Mills fields on $\partial \Omega$, provided that $i_{T} R^{D_{b}}=0$ and $i_{N} R^{D}=0$ on $H(\partial \Omega)$. If $S^{1} \rightarrow C(M) \stackrel{\pi}{\rightarrow} M$ is the canonical circle bundle and $\pi^{*} D$ is a Yang-Mills field with respect to the Fefferman metric $F_{\theta}$ of $(M, \theta)$ then $D$ is a pseudo Yang-Mills field on $M$. The Yang-Mills equations $\delta^{\pi^{*} D} R^{\pi^{*} D}=0$ project on the Euler-Lagrange equations $\delta_{b}^{D} R^{D}=0$ of the variational principle $\delta \mathcal{P Y \mathcal { M }}(D)=0$, provided that $i_{T} R^{D}=0$. When $M$ has vanishing pseudohermitian Ricci curvature the pullback $\pi^{*} D$ of the (CR invariant) Tanaka connection $D$ of $(E, h)$ is a Yang-Mills field on $C(M)$. We derive the second variation formula $\left\{d^{2} \mathcal{P} \mathcal{Y M}\left(D^{t}\right) / d t^{2}\right\}_{t=0}=$ $\int_{M}\left\langle\mathcal{S}_{b}^{D}(\varphi), \varphi\right\rangle \theta \wedge(d \theta)^{n}, D^{t}=D+A^{t}$ (provided that $D$ is a pseudo Yang-Mills field and $\left.\varphi \equiv\left\{d A^{t} / d t\right\}_{t=0} \in \operatorname{Ker}\left(\delta^{D}\right)\right)$, and show that $\mathcal{S}_{b}^{D}(\varphi) \equiv \Delta_{b}^{D} \varphi+\mathcal{R}_{b}^{D}(\varphi), \varphi \in \Omega^{0,1}(\operatorname{Ad}(E))$, is a subelliptic operator.
\end{abstract}

\section{INTRODUCTION}

A series of papers published in the last decade (J. Lewandowski \& P. Nurowski, [21], P. Nurowski, [24], P. Nurowski \& J. Tafel, [25]) 
are devoted to exploring the relationship among CR structures on 3dimensional manifolds and null solutions to Einstein equations, Maxwell equations, and Yang-Mills equations (cf. also J. Tafel, 28). Specifically, if $\left(M, T_{1,0}(M)\right)$ is a nondegenerate 3-dimensional CR manifold endowed with the contact form $\theta$ and with the (locally defined) complex 1-form $\theta^{1}$ such that $\theta^{1}\left(T_{1}\right)=1, \theta^{1}\left(T_{\overline{1}}\right)=0$ (where $T_{1}$ is a local generator of the CR structure $\left.T_{1,0}(M)\right)$ let us consider the semiRiemannian metric

$$
F=2 p^{2}\left\{\left(\pi^{*} \theta^{1}\right) \odot\left(\pi^{*} \theta^{\overline{1}}\right)-\left(\pi^{*} \theta\right) \odot \sigma\right\},
$$

on $M \times \mathbb{R}$, where $p$ is a real valued function on $M \times \mathbb{R}$ and $\sigma$ is a real 1-form on $M \times \mathbb{R}$ such that

$$
\pi^{*}\left(\theta \wedge \theta^{1} \wedge \theta^{\overline{1}}\right) \wedge \sigma \neq 0
$$

Here $\pi: M \times \mathbb{R} \rightarrow M$ is the projection. P. Nurowski has determined (cf. 24) local solutions to the Yang-Mills equations on $(M \times \mathbb{R}, F)$, under the additional assumption that the shear-free congruence of null geodesics tangent to $\partial / \partial \gamma$ ( $\gamma$ is the natural coordinate function on $\mathbb{R}$ ) possesses 3 linearly independent symmetries $\left\{X_{i}: 1 \leq i \leq 3\right\}$. Let $D$ be a $S U(3)$-connection in a vector bundle $\hat{E} \rightarrow M \times \mathbb{R}$ locally described by a matrix of 1 -forms $A=b \pi^{*} \theta^{1}+\bar{b} \pi^{*} \theta^{\overline{1}}+c \pi^{*} \theta+e \sigma$, where $a, b, c$ and $e$ are $\mathcal{G} \otimes \mathbb{C}$-valued functions $(\mathcal{G}=\mathbf{s u}(3))$ on $M \times \mathbb{R}$. When the Lie group $G_{3}$ (whose Lie algebra is generated by the $X_{i}$ 's) consists of symmetries of $D$ (i.e. each element of $G_{3}$ induces a gauge transformation of $A$ ) then (by a result of J. Harnad \& S. Shnider \& L. Vinet, [12]) up to some gauge transformation $A$ is strictly invariant under $G_{3}$. Then $\mathcal{L}_{X_{i}} A=0$, conditions which may be exploited to show that locally $D$ may be looked for in the form $A=B \pi^{*} \Omega^{1}+\bar{B} \pi^{*} \Omega^{\overline{1}}+C \pi^{*} \Omega$, with $B, C \in \mathcal{G} \otimes \mathbb{C}$. Here $\Omega$ and $\Omega^{1}$ are a new contact form and a new local coframe such that

$$
\mathcal{L}_{\tilde{X}_{i}} \Omega=0, \quad \mathcal{L}_{\tilde{X}_{i}} \Omega^{1}=0, \quad d \Omega=2 \sqrt{-1} \Omega^{1} \wedge \Omega^{\overline{1}},
$$

while $\tilde{X}_{i}$ are the projections on $M$ of the (nontrivial) symmetries $X_{i}$. Finally the Yang-Mills equations (for $S U(3)$-fields) on $M \times \mathbb{R}$ may be solved (together with the condition that $D$ is null, i.e. $d A+A \wedge A=$ $\left(\pi^{*} \Omega\right) \wedge\left(\Phi \pi^{*} \Omega^{1}+\bar{\Phi} \pi^{*} \Omega^{\overline{1}}\right)$, for some $\mathcal{G} \otimes \mathbb{C}$-valued function $\left.\Phi\right)$. For instance (cf. (5.10) in [24], p. 805)

$$
A=\rho \vec{n} \cdot f \pi^{*}\left(e^{i \phi} \Omega^{1}+e^{-i \phi} \Omega^{\overline{1}}\right)
$$

is a solution, where $\vec{n} \in \mathbb{R}^{3}$ is a unit vector, $\rho \in \mathbb{R}, \phi \in[0,2 \pi]$, and $f=\left(e_{1}, e_{2}, e_{3}\right)$ is a basis in $\mathcal{G}$. It is noteworthy that $\tilde{X}_{i}$ turn out to be symmetries of the CR structure $T_{1,0}(M)$ (in a sense that will be 
explained in section 3) and that the CR structures admitting the 3dimensional symmetry group $G_{3}$ are fully classified in 21, according to the Bianchi type of $G_{3}$. For instance, if $M=\left\{(x, y, z) \in \mathbb{R}^{3}: y \neq 0\right\}$ carries the CR structure $T_{1,0}(M)=\mathbb{C} T_{1}$ with

$$
T_{1}=\frac{y}{1+y^{2}} \frac{\partial}{\partial x}-\frac{i y}{2} \frac{\partial}{\partial y}+\frac{1}{y\left(1+y^{2}\right)} \frac{\partial}{\partial z}
$$

and the contact form $\theta=(1 / y) d x-y d z$ (such $M$ possesses a symmetry group of Bianchi type $V I_{0}$ ) then a local solution $A$ (to the Yang-Mills equations) of the form (3) may be produced. The example (4) of a CR structure on $\mathbb{R}^{3} \backslash\{y=0\}$ will be encountered again in section 3. Let now $M$ be a compact strictly pseudoconvex $\mathrm{CR}$ manifold, of arbitrary CR dimension $n$. Let $S^{1} \rightarrow C(M) \stackrel{\pi}{\longrightarrow} M$ be the canonical circle bundle (cf. section 3 for definitions). Note that $C(M)$ and $M \times \mathbb{R}$ are locally diffeomorphic. If $\theta$ is a contact form on $M$ then $C(M)$ carries a natural Lorentz metric $F_{\theta}$ (the Fefferman metric) and a moment's thought (compare to (68) in section 5) shows that when $M$ is 3-dimensional the Fefferman metric $F_{\theta}$ is of the form (11). Then, under the symmetry assumptions above, (3) is a (local) solution to the Yang-Mills equations

$$
\delta^{\mathbb{D}} R^{\mathbb{D}}=0
$$

on $\left(C(M), F_{\theta}\right)$ (with $n=1$ ), and in general it is conceivable that when the CR structure $T_{1,0}(M)$ possesses a symmetry group $G_{2 n+1}$, Nurowski's scheme may produce local symmetric null solutions to (5). A first step towards the achievement of this goal is performed in section 3. Note that (3) is the pullback (via $\pi$ ) to $M \times \mathbb{R}$ of a field on $M$. It is then a natural question whether given a Yang-Mills field on $\left(C(M), F_{\theta}\right)$ of the form $\pi^{*} D$, it follows that $D$ is a Yang-Mills field on $\left(M, g_{\theta}\right)$, where $g_{\theta}$ is the Webster metric. This question is answered in section 5 , where we integrate along the fibre in the Yang-Mills functional $\widehat{\mathcal{Y M}}$ on $C(M)$ and produce the new functional (7). As it turns out, $D$ is a pseudo Yang-Mills field (i.e. a critical point of (7)) rather than a YangMills field on $\left(M, g_{\theta}\right)$ (however, the two notions coincide in the special case $\left.i_{T} R^{D}=0\right)$. The converse (i.e. whether given a pseudo YangMills field $D$ on $M$ its pullback $\pi^{*} D$ is a Yang-Mills field on $C(M)$ ) is examined in Theorem 2, Solving (5) on $C(M)$ is therefore closely related to solving the pseudo Yang-Mills equations

$$
\delta_{b}^{D} R^{D}=0
$$


on $M$, and indeed (5) projects (under additional conditions, cf. section 5) on $M$ to give (6). One of the main results in this paper is that solutions to (6) occur as boundary values of Yang-Mills fields on a strictly pseudoconvex bounded domain $\Omega \subset \mathbb{C}^{n}$ endowed with the Bergman metric $g$ (cf. Theorem 11). For the proof of Theorem 2 we draw inspiration from [1] and make use of their canonical connection $\nabla$ (the Graham-Lee connection) whose pointwise restriction to a level set (near $\partial \Omega$ ) of a defining function of $\Omega$ is the better known Tanaka-Webster connection of the level set. Using the fine asymptotic properties of the Bergman kernel of $\Omega$ we may choose a defining function allowing an explicit relationship among the Bergman metric $g$ and the Webster metric of each level set, and therefore an explicit relationship among the Levi-Civita connection of $(\Omega, g)$ and the Graham-Lee connection. In the end, an elementary asymptotic analysis shows that boundary values $D_{b}$ of Yang-Mills fields $D$ on $(\Omega, g)$ satisfy (6) provided that $D_{b}$ satisfy certain compatibility conditions along $\partial \Omega$ (cf. section 4 ). In sections 6 and 7 we obtain the first and second variation formulae for the functional (7). The relevant operator occurring in the second variation formula is shown to be subelliptic of order $1 / 2$ (cf. Theorem 3). The problem of building an appropriate stability theory (along the lines of [4, yet relying on the subelliptic rather than on the elliptic theory) remains open. We feel that the importance of the Graham-Lee connection $\nabla$ in applications deserves Appendix A: there we provide a new axiomatic description of $\nabla$ together with a index-free proof.

\section{Statement of MAin Results}

Let $\left(M, T_{1,0}(M)\right)$ be a compact strictly pseudoconvex CR manifold, of CR dimension $n$, and $\theta$ a contact form on $M$. Let $\left(E, \bar{\partial}_{E}\right) \rightarrow M$ be a CR-holomorphic vector bundle and $h$ a Hermitian metric in $E$. Let $\mathcal{C}(E, h)$ be the affine space of all connections $D$ in $E$ such that $D h=0$. We consider the functional

$$
\mathcal{P Y \mathcal { M }}(D)=\frac{1}{2} \int_{M}\left\|\pi_{H} R^{D}\right\|^{2} \theta \wedge(d \theta)^{n} .
$$

Here $\pi_{H}: \Omega^{2}(\operatorname{Ad} E) \rightarrow \Omega^{2}(\operatorname{Ad} E) / \mathcal{J}_{\theta}^{2}$ is the natural projection and $\mathcal{J}_{\theta}^{\bullet}$ the ideal generated by $\theta$ in $\Omega^{\bullet}(\operatorname{Ad} E)$. A pseudo Yang-Mills field on $M$ is a critical point of $\mathcal{P} \mathcal{Y} \mathcal{M}: \mathcal{C}(E, h) \rightarrow[0,+\infty)$. We shall show that

Theorem 1. Let $\Omega=\{z \in U: \varphi(z)<0\}$ be a smoothly bounded strictly pseudoconvex domain in $\mathbb{C}^{n}$ and $g$ its Bergman metric. Let $\pi: F \rightarrow U$ be a holomorphic vector bundle and $h$ a Hermitian metric on $F$. Let $D_{b} \in \mathcal{C}(E, h)\left(E=\pi^{-1}(\partial \Omega)\right)$ be the boundary values of a 
Yang-Mills field $D \in \mathcal{C}(F, h)$ on $(\Omega, g)$. Assume that $i_{T} R^{D_{b}}=0$. Then $D_{b}$ is a pseudo Yang-Mills field if and only if $i_{N} R^{D}=0$ on $H(\partial \Omega)$.

Here $T$ is the characteristic direction of $(\partial \Omega, \theta), \theta \equiv \frac{i}{2}(\bar{\partial}-\partial) \varphi$, and $H(\partial \Omega)$ is the Levi distribution. Also $N=-J T$ ( $J$ is the complex structure on $\left.\mathbb{C}^{n}\right)$. The proof relies on the explicit relationship among the Levi-Civita connection $\nabla^{g}$ of $(\Omega, g)$ and the Graham-Lee connection $\nabla$ of $\varphi$ (cf. [11] and our Appendix A for the description and main properties of $\nabla)$.

Urakawa has started (cf. [30]-32] ) a study of Yang-Mills fields on $M$, that is of critical points of the functional

$$
\mathcal{Y} \mathcal{M}(D)=\frac{1}{2} \int_{M}\left\|R^{D}\right\|^{2} d \operatorname{vol}\left(g_{\theta}\right)
$$

where $d \operatorname{vol}\left(g_{\theta}\right)$ is the canonical volume form associated to the Webster metric $g_{\theta}$ of $(M, \theta)$. As it will be shortly shown, $\mathcal{Y} \mathcal{M}$ and $\mathcal{P} \mathcal{Y} \mathcal{M}$ are related. To motivate the definition of $\mathcal{P} \mathcal{Y} \mathcal{M}$ let $F_{\theta}$ be the Fefferman metric of $(M, \theta)$ (a Lorentz metric on $C(M)$, the total space of the canonical circle bundle $\pi: C(M) \rightarrow M$ (cf. e.g. J.M. Lee, [18])). By a result of E. Barletta et alt., [2, the base map $\phi: M \rightarrow N$ corresponding to any smooth $S^{1}$-invariant harmonic map $\Phi: C(M) \rightarrow N$ from $\left(C(M), F_{\theta}\right)$ into a Riemannian manifold $\left(N, g_{N}\right)$ is locally a subelliptic harmonic map (in the sense of J. Jost \& C-J. Xu, [15]). Also $\phi$ is a critical point of the functional $E(\phi)=\frac{1}{2} \int_{M} \operatorname{trace}_{G_{\theta}}\left(\pi_{H} \phi^{*} g_{N}\right) \theta \wedge(d \theta)^{n}$, where $G_{\theta}$ is the Levi form. Here, if $B$ is a bilinear form on $T(M)$ then $\pi_{H} B$ denotes the restriction of $B$ to $H(M)$, the Levi distribution of $\left(M, T_{1,0}(M)\right)$. The functional $E$ itself is obtained by integration along the fibre in the Dirichlet functional $\mathbb{E}(\Phi)=\frac{1}{2} \int_{C(M)} \operatorname{trace}_{F_{\theta}}\left(\Phi^{*} g_{N}\right) d \operatorname{vol}\left(F_{\theta}\right)$, where $\Phi=\phi \circ \pi$. Then perhaps subelliptic harmonic maps (rather than harmonic maps, with respect to the Webster metric) are the natural objects of study in CR geometry. Another example of the sort is the $C R$ Yamabe problem, i.e. given a contact form $\theta$ on $M$ such that $G_{\theta}$ is positive definite, find a contact form $\hat{\theta}=e^{u} \theta, u \in C^{\infty}(M)$, such that the pseudohermitian scalar curvature $\hat{\rho}$ of $(M, \hat{\theta})$ is a constant $\lambda$. By a result of J.M. Lee, [18, the Fefferman metric changes conformally $F_{\hat{\theta}}=e^{u \circ \pi} F_{\theta}$. Also the scalar curvature $K: C(M) \rightarrow \mathbb{R}$ of $\left(C(M), F_{\theta}\right)$ is $S^{1}$-invariant and the corresponding base function $\pi_{*} K: M \rightarrow \mathbb{R}$ is, up to a constant, the pseudohermitian scalar curvature $\rho$ of $(M, \theta)$ (precisely $\pi_{*} K=\frac{2 n+1}{n+1} \rho$ ). Therefore, the CR Yamabe problem is nothing but the Yamabe problem for the Fefferman metric and the relevant equation (the Yamabe equation on $\left(C(M), F_{\theta}\right)$ ) projects on $c_{n} \Delta_{b} u+\rho u=\lambda u^{p-1}$ (the CR Yamabe equation), a nonlinear subelliptic 
equation on $M$ (which may be analyzed with the techniques in [9], cf. D. Jerison \& J.M. Lee, 13-14, and N. Gamara \& R. Yacoub, 10, for a complete solution to the CR Yamabe problem). The common feature of the two examples above is that both provide natural objects on $M$, as projections of ( $S^{1}$-invariant) geometric quantities on $C(M)$, associated to the Fefferman metric. A more refined statement is that both examples lead to nonlinear subelliptic problems on $M$. This has been already emphasized for the CR Yamabe problem. As to the example of $S^{1}$-invariant harmonic maps $\Phi: C(M) \rightarrow N$, the base map is a solution to $\Delta_{b} \phi^{i}+g^{\alpha \bar{\beta}} T_{\alpha}\left(\phi^{j}\right) T_{\bar{\beta}}\left(\phi^{k}\right)\left(\left(\Gamma_{N}\right)_{j k}^{i} \circ \phi\right)=0$, where $\left(\Gamma_{N}\right)_{j k}^{i}$ are the Christoffel symbols of the second kind of $g_{N}$. On the same line of thought, we may state the following

Theorem 2. Let $M$ be a compact strictly pseudoconvex $C R$ manifold, of $C R$ dimension $n$. Let $\theta$ be a contact form on $M$ with $G_{\theta}$ positive definite. Let $\left(E, \bar{\partial}_{E}\right) \rightarrow M$ be a CR-holomorphic vector bundle and $h$ a Hermitian metric in $E$. $i$ ) There is a constant $c_{n}$ depending only on the dimension and the orientation of $M$ such that

(8) $c_{n} \mathcal{Y} \mathcal{M}(D)=\mathcal{P} \mathcal{Y} \mathcal{M}(D)+2 \int_{M}\left\|i_{T} R^{D}\right\|^{2} \theta \wedge(d \theta)^{n}, \quad D \in \mathcal{C}(E, h)$.

Consequently, given a Hermitian connection $D$ in $E$ whose curvature $R^{D}$ is of type $(1,1), D$ is a pseudo Yang-Mills field on $M$ if and only if $D$ is the Tanaka connection of $\left(E, \bar{\partial}_{E}, h\right)$. ii) Let $\widehat{\mathcal{Y M}}(\mathbb{D})=$ $\frac{1}{2} \int_{C(M)}\left\langle R^{\mathbb{D}}, R^{\mathbb{D}}\right\rangle d \operatorname{vol}\left(F_{\theta}\right)$ be the Yang-Mills functional on $C(M)$, for $\mathbb{D} \in \mathcal{C}\left(\pi^{*} E, \pi^{*} h\right)$. Then

$$
\widehat{\mathcal{Y M}}\left(\pi^{*} D\right)=2 \pi \mathcal{P} \mathcal{Y} \mathcal{M}(D), \quad D \in \mathcal{C}(E, h)
$$

Consequently, if $\pi^{*} D$ is a Yang-Mills field on $\left(C(M), F_{\theta}\right)$ then $D$ is a pseudo Yang-Mills field on $M$. Viceversa, let D be a pseudo Yang-Mills field on $M$ such that $i_{T} R^{D}=0$. Then $\pi^{*} D$ is a Yang-Mills field on $C(M)$ if and only if

$$
\left(R^{\alpha \bar{\beta}}-\frac{\rho}{2(n+1)} g^{\alpha \bar{\beta}}\right) R^{D}\left(T_{\alpha}, T_{\bar{\beta}}\right) u=0,
$$

for some local frame $\left\{T_{\alpha}: 1 \leq \alpha \leq n\right\}$ of $T_{1,0}(M)$ at any point $x \in M$, and

$$
\Lambda_{\theta} R^{D}=0 .
$$

In particular, if $M$ is (pseudohermitian) Ricci flat then the pullback $\pi^{*} D$ of the canonical Tanaka connection $D$ of $(E, h)$ is a Yang-Mills field. 
The main ingredients in the proof of Theorem 2 are a local coordinate calculation of the Fefferman metric of $(M, \theta)$, the explicit relationship among the Levi-Civita connection $\nabla^{C(M)}$ of $\left(C(M), F_{\theta}\right)$ and the Tanaka-Webster connection $\nabla$ of $(M, \theta)$ (cf. Lemma 2), and Theorem 2.3 in 30, p. 551. We may also state (delegating the definitions to section 2)

Theorem 3. Let $D$ be a pseudo Yang-Mills field and $D^{t}=D+A^{t}$, $|t|<\epsilon$, a smooth variation of $D$ whose first order part $\varphi \equiv\left\{d A^{t} / d t\right\}_{t=0}$ satisfies $i_{T} \varphi=0$ and $\delta_{b}^{D} \varphi=0$. Then

$$
\frac{d^{2}}{d t^{2}}\left\{\mathcal{P} \mathcal{Y} \mathcal{M}\left(D^{t}\right)\right\}_{t=0}=\int_{M}\left\langle\mathcal{S}_{b}^{D}(\varphi), \varphi\right\rangle \theta \wedge(d \theta)^{n}
$$

where $\mathcal{S}_{b}^{D}(\varphi) \equiv \Delta_{b}^{D} \varphi+\mathcal{R}_{b}^{D}(\varphi)$ and $\Delta_{b}^{D} \varphi \equiv d_{b}^{D} \delta_{b}^{D} \varphi+\delta_{b}^{D} d_{b}^{D} \varphi$ is the generalized sublaplacian. The operator $\mathcal{S}_{b}^{D}: \Omega^{0,1}(\operatorname{Ad}(E)) \rightarrow \Omega^{0,1}(\operatorname{Ad}(E))$ is subelliptic of order $1 / 2$.

As $\mathcal{R}_{b}^{D}$ is a zero order operator, the crucial point in the proof of Theorem 3 is to show that

$$
\begin{gathered}
\left(\Delta_{b}^{D} \varphi\right) \otimes e_{j}=2\left\{\square_{b} \varphi_{j}^{i}+\right. \\
\left.+(n-1)\left(\nabla_{T} \varphi_{j}^{i}+\varphi_{j}^{i} \circ \tau\right) \circ J\right\} \otimes e_{i}+\text { lower order terms }
\end{gathered}
$$

for any $\varphi \in \Omega^{0,1}(\operatorname{Ad}(E)), \varphi e_{j}=\varphi_{j}^{i} \otimes e_{i}$, and then exploit the subellipticity of the Kohn-Rossi operator $\square_{b}$ on scalar $(0,1)$-forms.

\section{CR AND PSEUDOHERMITIAN GEOMETRY}

3.1. Basic definitions and results. Let $M$ be a $C^{\infty}$ manifold, of real dimension $(2 n+1)$. A complex subbundle $T_{1,0}(M) \subset T(M) \otimes \mathbb{C}$, of complex rank $n$, is a $C R$ structure on $M$ (of $C R$ dimension $n$ ) if

$$
\begin{gathered}
T_{1,0}(M) \cap T_{0,1}(M)=(0), \\
Z, W \in \Gamma^{\infty}\left(T_{1,0}(M)\right) \Longrightarrow[Z, W] \in \Gamma^{\infty}\left(T_{1,0}(M)\right) .
\end{gathered}
$$

Here $T_{0,1}(M)=\overline{T_{1,0}(M)}$ is the complex conjugate of $T_{1,0}(M)$. Also, if $E \rightarrow M$ is a vector bundle then $\Gamma^{\infty}(E)$ denotes the space of $C^{\infty}$ sections in $E$ (eventually defined on some open set $U \subseteq M$, to be understood from the context). The tangential Cauchy-Riemann operator

$$
\bar{\partial}_{b}: C^{\infty}(M) \rightarrow \Gamma^{\infty}\left(T_{0,1}(M)^{*}\right)
$$

is given by $\left(\bar{\partial}_{b} f\right) \bar{Z}=\bar{Z}(f)$, for any $C^{\infty}$ function $f: M \rightarrow \mathbb{C}$ and any $Z \in T_{1,0}(M)$. Let $E \rightarrow M$ be a complex vector bundle over a CR manifold. A pre- $\bar{\partial}$-operator is a first order differential operator

$$
\bar{\partial}_{E}: \Gamma^{\infty}(E) \rightarrow \Gamma^{\infty}\left(T_{0,1}(M)^{*} \otimes E\right)
$$


such that

$$
\bar{\partial}_{E}(f u)=f \bar{\partial}_{E} u+\left(\bar{\partial}_{b} f\right) \otimes u,
$$

for any $f \in C^{\infty}(M)$ and any $u \in \Gamma^{\infty}(E)$. A pair $\left(E, \bar{\partial}_{E}\right)$ consisting of a complex vector bundle and a pre- $\bar{\partial}$-operator is a CR-holomorphic vector bundle if $\bar{\partial}_{E}$ satisfies the integrability condition

$$
[\bar{Z}, \bar{W}] \cdot u=\bar{Z} \cdot \bar{W} \cdot u-\bar{W} \cdot \bar{Z} \cdot u,
$$

for any $u \in \Gamma^{\infty}(E), Z, W \in T_{1,0}(M)$. Here $\bar{Z} \cdot u$ is short for $\left(\bar{\partial}_{E} u\right) \bar{Z}$.

Let $H(M)=\operatorname{Re}\left\{T_{1,0}(M) \oplus T_{0,1}(M)\right\}$ be the Levi distribution and $J: H(M) \rightarrow H(M), J(Z+\bar{Z})=i(Z-\bar{Z}), Z \in T_{1,0}(M)$, its complex structure $(i=\sqrt{-1})$. When $M$ is oriented, which is assumed throughout this paper, the conormal bundle $H(M)_{x}^{\perp}=\left\{\omega \in T_{x}^{*}(M)\right.$ : $\left.\operatorname{Ker}(\omega) \supseteq H(M)_{x}\right\}, x \in M$, is an oriented real line bundle, hence trivial $\left(H(M)^{\perp} \approx M \times \mathbb{R}\right.$, a vector bundle isomorphism). Therefore $H(M)^{\perp} \rightarrow M$ admits globally defined nowhere zero sections $\theta \in$ $\Gamma^{\infty}\left(H(M)^{\perp}\right)$, each of which is referred to as a pseudohermitian structure on $M$. The Levi form is

$$
L_{\theta}(Z, \bar{W})=-i(d \theta)(Z, \bar{W}),
$$

for any $Z, W \in T_{1,0}(M)$. $\left(M, T_{1,0}(M)\right)$ is nondegenerate if $L_{\theta}$ is nondegenerate for some $\theta$. If this is the case, each pseudohermitian structure $\theta$ is a contact form, i.e. $\theta \wedge(d \theta)^{n}$ is a volume form on $M$. Two pseudohermitian structures $\theta, \hat{\theta} \in \Gamma^{\infty}\left(H(M)^{\perp}\right)$ are related by $\hat{\theta}=f \theta$, for some $C^{\infty}$ function $f: M \rightarrow \mathbb{R} \backslash\{0\}$. Then $L_{\hat{\theta}}=f L_{\theta}$, hence nondegeneracy is a $\mathrm{CR}$ invariant notion (i.e. invariant under a transformation $\theta \mapsto f \theta$ of the pseudohermitian structure). Let $T$ is the unique nowhere zero globally defined tangent vector field on $M$, transverse to the Levi distribution, determined by $\theta(T)=1$ and $i_{T} d \theta=0$ (the characteristic direction of $d \theta$ ). Also, let us consider the semi-Riemannian metric $g_{\theta}$ (the Webster metric of $(M, \theta))$ given by

$$
g_{\theta}(X, Y)=G_{\theta}(X, Y), \quad g_{\theta}(X, T)=0, \quad g_{\theta}(T, T)=1,
$$

where $G_{\theta}(X, Y)=(d \theta)(X, J Y), X, Y \in H(M)$, is the (real) Levi form (note that $L_{\theta}$ and (the $\mathbb{C}$-linear extension of) $G_{\theta}$ coincide on $\left.T_{1,0}(M) \otimes T_{0,1}(M)\right)$. $\left(M, T_{1,0}(M)\right)$ is strictly pseudoconvex if $L_{\theta}$ is positive definite for some $\theta$. For instance, if $M=\left\{(x, y, u) \in \mathbb{R}^{3}: y \neq 0\right\}$ is endowed with the CR structure given by (4) in the Introduction then a calculation shows that the characteristic direction (corresponding to the contact form $\theta=(1 / y) d x-y d z)$ is

$$
T=\frac{y\left(3+y^{2}\right)}{4\left(1+y^{2}\right)} \frac{\partial}{\partial x}-\frac{i}{8} y\left(1-y^{2}\right) \frac{\partial}{\partial y}-\frac{1+3 y^{2}}{4 y\left(1+y^{2}\right)} \frac{\partial}{\partial z}
$$


so that

$$
\left[T_{1}, T_{\overline{1}}\right]=\frac{i}{2} T_{1}-\frac{i}{2} T_{\overline{1}}-\frac{2 i}{1+y^{2}} T
$$

(where $T_{\overline{1}}=\bar{T}_{1}$ ). Consequently

$$
L_{\theta}\left(T_{1}, T_{\overline{1}}\right)=(i / 2) \theta\left(\left[T_{1}, T_{\overline{1}}\right]\right)=1 /\left(1+y^{2}\right),
$$

hence $M$ is strictly pseudoconvex. A fundamental result in pseudohermitian geometry (established independently by N. Tanaka, 29, and S. Webster, 34]) is that on any nondegenerate CR manifold on which a contact form $\theta$ has been fixed there is a unique linear connection $\nabla$ (the Tanaka-Webster connection of $(M, \theta))$ such that i) $H(M)$ is parallel with respect to $\nabla$, ii) $\nabla g_{\theta}=0, \nabla J=0$, and iii) the torsion $T_{\nabla}$ of $\nabla$ is pure, i.e.

$$
\begin{gathered}
T_{\nabla}(Z, W)=0, T_{\nabla}(Z, \bar{W})=2 i L_{\theta}(Z, \bar{W}) T, \quad Z, W \in T_{1,0}(M), \\
\tau \circ J+J \circ \tau=0,
\end{gathered}
$$

where $\tau(X)=T_{\nabla}(T, X), X \in T(M)$, is the pseudohermitian torsion. If $M$ is 3 -dimensional $(n=1)$ and $T_{1}$ is a local generator of the $\mathrm{CR}$ structure we set

$$
\nabla_{T_{1}} T_{1}=\Gamma_{11}^{1} T_{1}, \quad \nabla_{T_{\overline{1}}} T_{1}=\Gamma_{\overline{1} 1}^{1} T_{1}, \quad \nabla_{T} T_{1}=\Gamma_{01}^{1} T_{1} .
$$

A calculation (based on (i)-(iii)) shows that

$$
\begin{gathered}
\Gamma_{11}^{1}=g^{1 \overline{1}}\left\{T_{1}\left(g_{1 \overline{1}}\right)-g_{\theta}\left(T_{1},\left[T_{1}, T_{\overline{1}}\right]\right)\right\}, \\
\Gamma_{\overline{1} 1}^{1}=g^{1 \overline{1}} g_{\theta}\left(\left[T_{\overline{1}}, T_{1}\right], T_{\overline{1}}\right), \\
\Gamma_{01}^{1}=g^{1 \overline{1}} g_{\theta}\left(\left[T, T_{1}\right], T_{\overline{1}}\right) .
\end{gathered}
$$

Here $g_{1 \overline{1}}=L_{\theta}\left(T_{1}, T_{\overline{1}}\right)$ and $g^{1 \overline{1}}=1 / g_{1 \overline{1}}$. Going back to the example $\mathbb{R}^{3} \backslash\{y=0\}$ with the CR structure (4) we have

$$
\left[T, T_{1}\right]=\frac{i}{8}\left(1-y^{2}\right) T_{1}+\frac{i}{8}\left(1+y^{2}\right) T_{\overline{1}}
$$

hence (by (14)- (16) )

$$
\Gamma_{11}^{1}=i\left(\frac{1}{2}+\frac{y^{2}}{1+y^{2}}\right), \quad \Gamma_{\overline{1} 1}^{1}=-\frac{i}{2}\left(1+y^{2}\right), \quad \Gamma_{01}^{1}=\frac{i}{8}\left(1-y^{2}\right) .
$$

We assume from now on that, unless otherwise stated, $M$ is strictly pseudoconvex. A complex valued differential $p$-form $\eta$ on $M$ is of type $(p, 0)$ (or a $(p, 0)$-form on $M)$ if $\left.T_{0,1}(M)\right\rfloor \eta=0$. Let $\theta$ be a contact form on $M$ and $T$ the characteristic direction of $d \theta$. Let $\left\{T_{\alpha}: 1 \leq \alpha \leq n\right\}$ be a local frame in $T_{1,0}(M)$, defined on an open set $U \subseteq M$. Let $\left\{\theta^{\alpha}\right.$ : 
$1 \leq \alpha \leq n\}$ be the corresponding admissible coframe, i.e, the (locally defined) complex 1-forms determined by $\theta^{\alpha}\left(T_{\beta}\right)=\delta_{\beta}^{\alpha}, \theta^{\alpha}\left(T_{\bar{\beta}}\right)=0$, and $\theta^{\alpha}(T)=0$. Here $T_{\bar{\beta}}=\overline{T_{\beta}}$. Then $\left\{\theta^{\alpha}, \theta^{\bar{\alpha}}, \theta\right\}$ is a (local) frame of $T^{*}(M) \otimes \mathbb{C}$ on $U$ and a $(p, 0)$-form $\eta$ on $M$ may be locally expressed as sums of monomials of the form $\theta^{\alpha_{1}} \wedge \cdots \wedge \theta^{\alpha_{p}}$ or $\theta \wedge \theta^{\alpha_{1}} \wedge \cdots \wedge \theta^{\alpha_{p-1}}$ (with $C^{\infty}(U)$-coefficients). Therefore, the top degree complex forms $\eta$ such that $\left.T_{1,0}(M)\right\rfloor \eta=0$ are (unlike the case of complex manifolds, where the top degree is the complex dimension) the forms of type $(n+1,0)$ (where $n$ is the CR dimension). Let $K(M)=\Lambda^{n+1,0}(M) \rightarrow M$ be the bundle of $(n+1,0)$-forms on $M$ (the canonical line bundle). There is a natural action of $\mathbb{R}^{+}=(0,+\infty)$ on $K(M) \backslash\{$ zero section $\}$. Let $C(M)$ be the quotient space and $\pi: C(M) \rightarrow M$ the projection. Then $C(M) \rightarrow M$ is a principal $S^{1}$-bundle (the canonical circle bundle). Its locally trivial structure is described by

$$
\begin{gathered}
\pi^{-1}(U) \rightarrow U \times S^{1}, \quad[\omega] \mapsto(x, \lambda /|\lambda|), \\
\omega=\lambda\left(\theta \wedge \theta^{1} \wedge \cdots \wedge \theta^{n}\right)_{x}, x \in U, \lambda \in \mathbb{C}^{*}=\mathbb{C} \backslash\{0\} .
\end{gathered}
$$

We shall need the local fibre coordinate

$$
\gamma: \pi^{-1}(U) \rightarrow \mathbb{R}, \quad \gamma([\omega])=\arg (\lambda /|\lambda|),
$$

where $\arg : S^{1} \rightarrow[0,2 \pi)$. Let $\left(E, \bar{\partial}_{E}\right) \rightarrow M$ be a CR-holomorphic vector bundle. Let $h$ be a Hermitian metric in $E$. Let $\mathcal{C}(E, h)$ be the affine space of all connections $D$ in $E$ such that $D h=0$, i.e.

$$
X(h(u, v))=h\left(D_{X} u, v\right)+h\left(u, D_{\bar{X}} v\right),
$$

for any $X \in T(M) \otimes \mathbb{C}$ and any $u, v \in \Gamma^{\infty}(E)$. A connection $D \in$ $\mathcal{C}(E, h)$ is Hermitian if $D^{0,1}=\bar{\partial}_{E}$. Here $D^{0,1} u$ is the restriction of $D u$ to $T_{0,1}(M)$. Let $\operatorname{Ad}(E) \rightarrow M$ be the subbundle of $\operatorname{End}(E) \rightarrow M$ consisting of all skew-symmetric endomorphisms $S$, i.e. $h(S u, v)+$ $h(u, S v)=0$, for any $u, v \in \Gamma^{\infty}(E)$. By a result in [6], p. 43, given a contact form $\theta$ and an endomorphism $S \in \Gamma^{\infty}(\operatorname{Ad}(E))$ there is a unique Hermitian connection $D=D(h, \theta, S)$ in $E$ (the canonical $S$ connection) such that

$$
\Lambda_{\theta} R^{D}=2 n S \text {. }
$$

Here $R^{D}=D \circ D: \Omega^{0}(E) \rightarrow \Omega^{2}(E)$ is the curvature 2-form of $D$. Also we set $\Omega^{k}(E)=\Gamma^{\infty}\left(\Lambda^{k} T^{*}(M) \otimes E\right), k \geq 0$. If $F \rightarrow M$ is a vector bundle and $\varphi \in \Gamma^{\infty}\left(T^{*}(M) \otimes T^{*}(M) \otimes F\right)$ the trace $\Lambda_{\theta} \varphi$ of $\varphi$ is given by

$$
i\left(\Lambda_{\theta} \varphi\right)_{x}=\sum_{\alpha=1}^{n} \varphi\left(Z_{\alpha}, Z_{\bar{\alpha}}\right)_{x}
$$


where $\left\{Z_{\alpha}\right\}$ is a (local) orthonormal (i.e. $L_{\theta}\left(Z_{\alpha}, Z_{\bar{\beta}}\right)=\delta_{\alpha \beta}$ ) frame of $T_{1,0}(M)$ on $U \ni x$. Therefore $\Lambda_{\theta} \varphi \in \Gamma^{\infty}(F)$. When $S=0$ the canonical $S$-connection is the Tanaka connection $D(h, \theta, 0)$ in $E \rightarrow M$ (cf. 29]). $D(h, \theta, 0)$ is a $\mathrm{CR}$ invariant. Assume $M$ to be compact. The Yang-Mills functional $\mathcal{Y} \mathcal{M}: \mathcal{C}(E, h) \rightarrow[0,+\infty)$ is given by

$$
\mathcal{Y} \mathcal{M}(D)=\frac{1}{2} \int_{M}\left\|R^{D}\right\|^{2} \theta \wedge(d \theta)^{n}
$$

A Yang-Mills field on $M$ is a critical point $D \in \mathcal{C}(E, h)$ of $\mathcal{Y} \mathcal{M}$, i.e. a solution to the Yang-Mills equations

$$
\delta^{D} R^{D}=0 .
$$

Let $\Omega$ be a differential 2 -form on $M$. Then $\Omega$ is of type $(1,1)$ if $\Omega(Z, W)=0, \Omega(\bar{Z}, \bar{W})=0$, for any $Z, W \in T_{1,0}(M)$, and $i_{T} \Omega=0$. Let $D \in \mathcal{C}(E, h)$ be a Hermitian connection such that its curvature $R^{D}$ is a form of type $(1,1)$. By a result in [30, $D$ is a Yang-Mills field if and only if $D$ is the Tanaka connection $D(h, \theta, 0)$. In general, canonical $S$ connections solve the inhomogeneous Yang-Mills equations $\delta^{D} R^{D}=f$, in the presence of suitable compatibility conditions satisfied by $f$ (cf. Theorem 2 in [6], p. 44-45).

3.2. Symmetric CR structures. The CR structure $T_{1,0}(M)$ is symmetric if there is $X \in \mathcal{X}(M)$ such that

$$
\mathcal{L}_{X} \theta=t \theta, \quad \mathcal{L}_{X} \theta^{\alpha}=w_{\beta}^{\alpha} \theta^{\beta}+\ell^{\alpha} \theta
$$

for some functions $t, w_{\beta}^{\alpha}, \ell^{\alpha}$ on $M$ ( $t$ real valued) and $X$ is a symmetry of $T_{1,0}(M)$. If

$$
\hat{\theta}=e^{u} \theta, \quad \hat{\theta}^{\alpha}=U_{\beta}^{\alpha} \theta^{\beta}+v^{\alpha} \theta,
$$

(where $\left[U_{\beta}^{\alpha}\right]$ is $\mathrm{GL}(n, \mathbb{C})$-valued) and $X$ is a symmetry of the CR structure then

$$
\begin{gathered}
\mathcal{L}_{X} \hat{\theta}^{\alpha}=\hat{w}_{\beta}^{\alpha} \hat{\theta}^{\beta}+\hat{\ell}^{\alpha} \hat{\theta} \\
\hat{w}_{\beta}^{\alpha} \equiv\left(U^{-1}\right)_{\beta}^{\gamma}\left\{X\left(U_{\gamma}^{\alpha}\right)+U_{\rho}^{\alpha} w_{\gamma}^{\rho}\right\}, \\
\hat{\ell}^{\alpha} \equiv e^{-u}\left\{X\left(v^{\alpha}\right)+U_{\beta}^{\alpha} \ell^{\beta}+v^{\alpha} t-\left(U^{-1}\right)_{\rho}^{\gamma} v^{\rho}\left[X\left(U_{\gamma}^{\alpha}\right)+U_{\beta}^{\alpha} w_{\gamma}^{\beta}\right]\right\} .
\end{gathered}
$$

In particular (19)-(20) show that the notion of symmetric CR structure is globally defined. Assume from now on that the CR structure $T_{1,0}(M)$ admits $2 n+1$ linearly independent symmetries $\tilde{X}_{1}, \cdots, \tilde{X}_{2 n+1} \in \mathcal{X}(M)$ such that $\left[\tilde{X}_{i}, \tilde{X}_{j}\right]=c_{i j}^{k} \tilde{X}_{k}$, for some $c_{i j}^{k} \in \mathbb{R}$. 
Proposition 1. (P. Nurowski, [24])

Let $M$ be a strictly pseudoconvex $C R$ manifold with $H^{1}(M ; \mathbb{R})=0$. There is a transformation $\left\{\theta, \theta^{\alpha}\right\} \mapsto\left\{\Omega, \Omega^{\alpha}\right\}$ of the form

$$
\Omega=e^{u} \theta, \quad \Omega^{\alpha}=U_{\beta}^{\alpha} \theta^{\beta}+v^{\alpha} \theta,
$$

where $\left[U_{\beta}^{\alpha}\right]$ is $\mathrm{GL}(n, \mathbb{C})$-valued, such that

$$
\mathcal{L}_{\tilde{X}_{i}} \Omega=0, \quad \mathcal{L}_{\tilde{X}_{i}} \Omega^{\alpha}=0, \quad 1 \leq i \leq 2 n+1 .
$$

Here $H^{1}(M ; \mathbb{R})$ is the first de Rham cohomology group. Its vanishing guarantees that the solution $u$ to (22) is globally defined.

Proof of Proposition 2. As $\tilde{X}_{i}$ are symmetries of the CR structure

$$
\mathcal{L}_{\tilde{X}_{i}} \theta=t_{i} \theta, \quad \mathcal{L}_{\tilde{X}_{i}} \theta^{\alpha}=w_{i \beta}^{\alpha} \theta^{\beta}+\ell_{i}^{\alpha} \theta .
$$

We must solve the system of first order linear PDEs

$$
\begin{gathered}
t_{i}+\tilde{X}_{i}(u)=0, \\
\tilde{X}_{i}\left(U_{\beta}^{\alpha}\right)+U_{\gamma}^{\alpha} w_{i \beta}^{\gamma}=0, \\
\tilde{X}_{i}\left(v^{\alpha}\right)+U_{\beta}^{\alpha} \ell_{i}^{\beta}+v^{\alpha} t_{i}=0,
\end{gathered}
$$

with the unknowns $u, U_{\beta}^{\alpha}$ and $v^{\alpha}$. Let $\eta \in \Omega^{1}(M)$ be defined by $\eta\left(\tilde{X}_{i}\right)=t_{i}, 1 \leq i \leq 2 n+1$. Then (22) may be written $d u+\eta=0$. We have

$$
\mathcal{L}_{\tilde{X}_{i}} \mathcal{L}_{\tilde{X}_{j}} \theta=\mathcal{L}_{\tilde{X}_{j}} \mathcal{L}_{\tilde{X}_{i}} \theta+\mathcal{L}_{\left[\tilde{X}_{i}, \tilde{X}_{j}\right]} \theta
$$

hence

$$
\tilde{X}_{i}\left(t_{j}\right)-\tilde{X}_{j}\left(t_{i}\right)-c_{i j}^{k} t_{k}=0
$$

that is $d \eta=0$. Thus there is a globally defined real valued function $g \in C^{\infty}(M)$ such that $\eta=d g$ and $u \equiv-g$ solves (22). Next, we consider the (locally defined) 1 -forms $\eta_{\beta}^{\alpha}$ and $\eta^{\alpha}$ given by

$$
\eta_{\beta}^{\alpha}\left(\tilde{X}_{i}\right)=w_{i \beta}^{\alpha}, \quad \eta^{\alpha}\left(\tilde{X}_{i}\right)=\ell_{i}^{\alpha}, \quad 1 \leq i \leq 2 n+1 .
$$

Then (23) $-(24)$ may be written

$$
\begin{gathered}
d U_{\beta}^{\alpha}+U_{\gamma}^{\alpha} \eta_{\beta}^{\gamma}=0, \\
d v^{\alpha}+v^{\alpha} \eta+U_{\beta}^{\alpha} \eta^{\beta}=0 .
\end{gathered}
$$


Assuming that (25) has been solved in a neighborhood $U$ of each point, let us solve (26). Multiplying in both sides by $e^{-u}$ (where $u$ is a solution to (22)) leads to

$$
d\left(e^{-u} v^{\alpha}\right)+e^{-u} U_{\beta}^{\alpha} \eta^{\beta}=0 .
$$

Therefore, to prove existence of a (local) solution $v^{\alpha}$ to (26) it suffices to show that $e^{-u} U_{\beta}^{\alpha} \eta^{\beta}$ is exact (in a neighborhood of a point). The identity

$$
\mathcal{L}_{\tilde{X}_{i}} \mathcal{L}_{\tilde{X}_{j}} \theta^{\alpha}=\mathcal{L}_{\tilde{X}_{j}} \mathcal{L}_{\tilde{X}_{i}} \theta^{\alpha}+\mathcal{L}_{\left[\tilde{X}_{i}, \tilde{X}_{j}\right]} \theta^{\alpha}
$$

yields

$$
\begin{gathered}
d \eta_{\beta}^{\alpha}=\eta_{\gamma}^{\alpha} \wedge \eta_{\beta}^{\gamma}, \\
d \eta^{\alpha}=\eta_{\beta}^{\alpha} \wedge \eta^{\beta}+\eta^{\alpha} \wedge \eta .
\end{gathered}
$$

Let $U_{\beta}^{\alpha}$ be a solution to (25). Then (by (28)

$$
\begin{gathered}
d\left(e^{-u} U_{\beta}^{\alpha} \eta^{\beta}\right)=e^{-u}\left\{d U_{\beta}^{\alpha} \wedge \eta^{\beta}+U_{\beta}^{\alpha} d \eta^{\beta}-U_{\beta}^{\alpha} d u \wedge \eta^{\beta}\right\}= \\
=e^{-u} U_{\beta}^{\alpha}\left\{d \eta^{\beta}-\eta_{\gamma}^{\beta} \wedge \eta^{\gamma}-\eta^{\beta} \wedge \eta\right\}=0 .
\end{gathered}
$$

Thus there is a function $f^{\alpha} \in C^{\infty}(U)$ such that $e^{-u} U_{\beta}^{\alpha} \eta^{\beta}=d f^{\alpha}$ and $v^{\alpha} \equiv-e^{u} f^{\alpha}$ solves (26). To solve (25) let $\left(U, x^{i}\right)$ be a normal coordinate neighborhood at a point $x_{0} \in M$ (we think of $M$ as a Riemannian manifold with the Webster metric $g_{\theta}$ ). We shall show that for any $c_{\beta}^{\alpha} \in \mathbb{C}$ there is a unique solution to (25) with the initial condition $U_{\beta}^{\alpha}\left(x_{0}\right)=c_{\beta}^{\alpha}$. Let $a=\left(a^{1}, \cdots, a^{2 n+1}\right) \in U$ be an arbitrary point and let us consider the geodesic $a_{t}=\left(a^{1} t, \cdots, a^{2 n+1} t\right)$. Let $f_{\beta}^{\alpha}(t)$ be the solution to the Cauchy problem for the system of ODEs

$$
\frac{d f_{\beta}^{\alpha}}{d t}+f_{\gamma}^{\alpha}(t) \eta_{\beta}^{\gamma}\left(\dot{a}_{t}\right)=0
$$

with the initial condition $f_{\beta}^{\alpha}(0)=c_{\beta}^{\alpha}$, where $\dot{a}_{t}$ is the tangent vector at $a_{t}$. We define $U_{\beta}^{\alpha} \in C^{\infty}(U)$ by setting $U_{\beta}^{\alpha}(a)=f_{\beta}^{\alpha}(1)$. Of course, if we start with $\operatorname{det}\left(c_{\beta}^{\alpha}\right) \neq 0$ then $\left[U_{\beta}^{\alpha}\right]$ is $\operatorname{GL}(m, \mathbb{C})$-valued on a neighborhood of $x_{0}$. We wish to show that $U_{\beta}^{\alpha}$ satisfies (25), i.e.

$$
Y\left(U_{\beta}^{\alpha}\right)+U_{\gamma}^{\alpha}(a) \eta_{\beta, a}^{\gamma}(Y)=0
$$

for any $Y=b^{j}\left(\partial / \partial x^{j}\right)_{a} \in T_{a}(M)$. We start by extending $Y$ to the vector field $Y=b^{j} \partial / \partial x^{j}$ with constant components $b^{j}$ on $U$. Similarly, let us extend the vector field $\dot{a}_{t}$ along the geodesic $a_{t}$ to the vector field $X=a^{i} \partial / \partial x^{i}$. We shall show that along $a_{t}$

$$
X\left(Y\left(U_{\beta}^{\alpha}\right)+U_{\rho}^{\alpha} \eta_{\beta}^{\rho}(Y)\right)+\left(Y\left(U_{\gamma}^{\alpha}\right)+U_{\rho}^{\alpha} \eta_{\gamma}^{\rho}(Y)\right) \eta_{\beta}^{\gamma}(X)=0 .
$$


When this is done, we see that $Y\left(U_{\beta}^{\alpha}\right)+U_{\rho}^{\alpha} \eta_{\beta}^{\rho}(Y)$, clearly satisfying $Y\left(U_{\beta}^{\alpha}\right)+U_{\rho}^{\alpha} \eta_{\beta}^{\rho}(Y)=0$ at $x_{0}$, must be the zero function (which satisfies (30) with the same initial condition), i.e. (29) is verified. It remains that we prove (30). This follows from (27). Indeed (as $[X, Y]=0$ )

$$
X\left(\eta_{\beta}^{\alpha}(Y)\right)=Y\left(\eta_{\beta}^{\alpha}(X)\right)+\eta_{\gamma}^{\alpha}(X) \eta_{\beta}^{\gamma}(Y)-\eta_{\gamma}^{\alpha}(Y) \eta_{\beta}^{\gamma}(X)
$$

and the proof of (30) is straightforward. Proposition 1 is a first step towards recovering the methods of P. Nurowski, 24] [eventually leading to local solutions of the Yang-Mills equations on $\left.\left(C(M), F_{\theta}\right)\right]$ as mentioned in the Introduction. The result in Proposition 1 may be refined to show that there is a coframe $\left\{\Omega, \Omega^{\alpha}\right\}$ such that

$$
\mathcal{L}_{\tilde{X}_{i}} \Omega=0, \quad \mathcal{L}_{\tilde{X}_{i}} \Omega^{\alpha}=0, d \Omega=2 i \sum_{\alpha=1}^{n} \Omega^{\alpha} \wedge \Omega^{\bar{\alpha}}
$$

(compare to (2) in the Introduction). The proof is illustrative of the local methods in pseudohermitian geometry. Let $\left\{\Omega, \Omega^{\alpha}\right\}$ be the 1forms furnished by Proposition 1 given by a transformation of the form (21). If $\left\{T, T_{\alpha}\right\}$ is such that $\theta(T)=1, i_{T} d \theta=0$ and $\theta^{\alpha}\left(T_{\beta}\right)=\delta_{\beta}^{\alpha}$, $\theta^{\alpha}\left(T_{\bar{\beta}}\right)=0, \theta^{\alpha}(T)=0$, let us set $W_{\alpha}=\left(U^{-1}\right)_{\alpha}^{\beta} T_{\beta}$. One may easily show that

$$
d \Omega=2 i G_{\alpha \bar{\beta}} \Omega^{\alpha} \wedge \Omega^{\bar{\beta}}+\Phi \wedge \Omega,
$$

where $G_{\alpha \bar{\beta}}=e^{u}\left(U^{-1}\right)_{\alpha}^{\gamma} U_{\bar{\beta}}^{\bar{\rho}} g_{\gamma \bar{\rho}}$ and $\Phi=e^{-u}\left\{W_{\alpha}(u) \Omega^{\alpha}+W_{\bar{\alpha}}(u) \Omega^{\bar{\alpha}}\right\}$. By $\mathcal{L}_{X}=d \circ i_{X}+i_{X} \circ d$ it follows that $\mathcal{L}_{\tilde{X}_{i}} d \Omega=0$. Taking the Lie derivative of (31) gives

$$
0=\mathcal{L}_{\tilde{X}_{i}} d \Omega=2 \sqrt{-1} \mathcal{L}_{\tilde{X}_{i}}\left(G_{\alpha \bar{\beta}}\right) \Omega^{\alpha} \wedge \Omega^{\bar{\beta}}+\left(\mathcal{L}_{\tilde{X}_{i}} \Phi\right) \wedge \Omega
$$

hence $\left(\operatorname{as} \mathcal{L}_{\tilde{X}_{i}} \Phi \equiv 0, \bmod \Omega\right.$ ) on one hand $\tilde{X}_{i}\left(G_{\alpha \bar{\beta}}\right)=0$, i.e. $G_{\alpha \bar{\beta}}=$ $a_{\alpha \bar{\beta}} \in \mathbb{C}$, and on the other $\mathcal{L}_{\tilde{X}_{i}} \Phi=0$. The latter may be written $d u_{\alpha}-u_{\alpha} d u=0$ (where $u_{\alpha}=W_{\alpha}(u)$ ). Hence $d\left(e^{-u} u_{\alpha}\right)=0$, i.e. $u_{\alpha}=c_{\alpha} e^{u}$, for some $c_{\alpha} \in \mathbb{C}$. Therefore $\Phi=c_{\alpha} \Omega^{\alpha}+c_{\bar{\alpha}} \Omega^{\bar{\alpha}}$. Finally, let $\left[b_{\beta}^{\alpha}\right]$ be a square root of $\left[a_{\alpha \bar{\beta}}\right]$ (as $\left[a_{\alpha \bar{\beta}}\right]$ is positive definite) and consider the transformation

$$
\hat{\Omega}^{\alpha}=b_{\beta}^{\alpha} \Omega^{\beta}+\frac{i}{2} c_{\bar{\beta}}\left(b^{-1}\right)_{\bar{\alpha}}^{\bar{\beta}} \Omega .
$$

Then $d \Omega=2 i \sum_{\alpha=1}^{n} \hat{\Omega}^{\alpha} \wedge \hat{\Omega}^{\bar{\alpha}}$ and $\mathcal{L}_{\tilde{X}_{i}} \hat{\Omega}^{\alpha}=0$. Q.e.d.

\section{Boundary VAlues of YANG-Mills FieldS}

Let $\Omega \subset \mathbb{C}^{n}$ be a bounded domain with smooth boundary $\partial \Omega$, i.e. there is a neighborhood $U \supset \bar{\Omega}$ and a real valued function $\varphi \in C^{\infty}(U)$ 
such that $\Omega=\{z \in U: \varphi(z)<0\}, \partial \Omega=\{z \in U: \varphi(z)=0\}$, and $\nabla \varphi(z) \neq 0$, for any $z \in \partial \Omega$. We assume that $\Omega$ is strictly pseudoconvex, i.e. $\partial \Omega$ is a strictly pseudoconvex $\mathrm{CR}$ manifold (with the natural $\mathrm{CR}$ structure $T_{1,0}(\partial \Omega)=T^{1,0}\left(\mathbb{C}^{n}\right) \cap[T(\partial \Omega) \otimes \mathbb{C}]$ induced by the complex structure of the ambient space).

Let $\pi: F \rightarrow U$ be a holomorphic vector bundle. The portion $E=$ $\pi^{-1}(\partial \Omega)$ of $F$ over the boundary of $\Omega$ is CR-holomorphic. Indeed, as $F$ is holomorphic, there is a natural differential operator

$$
\bar{\partial}_{F}: \Gamma^{\infty}(F) \rightarrow \Gamma^{\infty}\left(T^{0,1}(U)^{*} \otimes F\right)
$$

where $T^{0,1}(U)$ is the anti-holomorphic tangent bundle over $U$. Given $u \in \Gamma^{\infty}(E)$ let $\tilde{u} \in \Gamma^{\infty}(F)$ be a $C^{\infty}$ extension of $u$ as a cross-section in $F$ and set $\left(\bar{\partial}_{E} u\right)_{z}=\left(\bar{\partial}_{F} \tilde{u}\right)_{z}$ for any $z \in \partial \Omega$. The definition of $\left(\bar{\partial}_{E} u\right)_{z}$ does not depend upon the choice of extension $\tilde{u}$ of $u$ because $\left.(\bar{\partial} f)\right|_{T_{0,1}(\partial \Omega)}=\bar{\partial}_{b}\left(\left.f\right|_{\partial \Omega}\right)$ for any $C^{\infty}$ function $f: U \rightarrow \mathbb{C}$. Let $\left\{\Phi_{\alpha}\right.$ : $\left.\pi^{-1}\left(\Omega_{\alpha}\right) \rightarrow \Omega_{\alpha} \times \mathbb{C}^{m}: \alpha \in I\right\}$ be a trivialization atlas for $F$ and $G_{\beta \alpha}: \Omega_{\beta} \cap \Omega_{\alpha} \rightarrow \mathrm{GL}(m, \mathbb{C})$ the corresponding transition functions. Set $U_{\alpha}=\Omega_{\alpha} \cap \partial \Omega$ and $g_{\beta \alpha}=\left.G_{\beta \alpha}\right|_{U_{\alpha} \cap U_{\beta}}$. As $G_{\beta \alpha}$ are holomorphic, it follows that $E \rightarrow \partial \Omega$ is a peculiar type of CR-holomorphic vector bundle (called locally trivial by C. Le Brun, 17]) in that its transition functions $g_{\beta \alpha}$ are matrix valued CR functions on $\partial \Omega$.

Let $K(\zeta, z)$ be the Bergman kernel of $\Omega$. By a classical result in 8

$$
K(\zeta, z)=c_{\Omega}|\nabla \varphi(z)|^{2} \cdot \operatorname{det} L_{\varphi}(z) \cdot \Psi(\zeta, z)^{-(n+1)}+H(\zeta, z),
$$

(the Fefferman asymptotic expansion formula for the Bergman kernel) where $H \in C^{\infty}(\bar{\Omega} \times \bar{\Omega} \backslash \Delta), \Delta$ is the diagonal of $\partial \Omega \times \partial \Omega$, and $H$ satisfies the estimate

$$
|H(\zeta, z)| \leq c_{\Omega}^{\prime}|\Psi(\zeta, z)|^{-(n+1)+1 / 2} \cdot|\log | \Psi(\zeta, z)|| .
$$

Here $L_{\varphi}=\partial \bar{\partial} \varphi$. Also we set

$$
\Psi(\zeta, z)=(F(\zeta, z)-\varphi(z)) \chi(|\zeta-z|)+(1-\chi(|\zeta-z|))|\zeta-z|^{2}
$$

where

$$
F(\zeta, z)=-\sum_{j=1}^{n} \frac{\partial \varphi}{\partial z^{j}}(z)\left(\zeta^{j}-z^{j}\right)-\frac{1}{2} \sum_{j, k=1}^{n} \frac{\partial^{2} \varphi}{\partial z^{j} \partial z^{k}}(z)\left(\zeta^{j}-z^{j}\right)\left(\zeta^{k}-z^{k}\right)
$$

and $\chi(t)$ is a $C^{\infty}$ cut-off function with $\chi(t)=1$ for $|t|<\epsilon_{0} / 2$ and $\chi(t)=0$ for $|t| \geq 3 \epsilon_{0} / 4$. As a consequence of (32)

$$
K(z, z)^{-1 /(n+1)}=|\varphi(z)|\left(\Phi(z)+H(z, z)|\varphi(z)|^{n+1}\right)^{-1 /(n+1)}
$$


where $\Phi(z) \equiv c_{\Omega}|\nabla \varphi(z)|^{2} \operatorname{det} L_{\varphi}(z)$ stays finite near $\partial \Omega$ and (by (33))

$$
|H(z, z)||\varphi(z)|^{n+1} \leq c_{\Omega}^{\prime}|\varphi(z)|^{1 / 2}|\log | \varphi(z) \| \rightarrow 0, \quad \text { as } z \rightarrow \partial \Omega .
$$

Therefore $K(z, z)^{-1 /(n+1)}$ vanishes at $\partial \Omega$. Also, as $\Phi(z) \neq 0$ near the boundary, $\nabla K(z, z)^{-1 /(n+1)} \neq 0$ along $\partial \Omega$, hence $K(z, z)^{-1 /(n+1)}$ may be used as a defining function for $\Omega$.

For the rest of this section we assume that $\varphi(z) \equiv-K(z, z)^{-1 /(n+1)}$ and set $\theta \equiv \frac{i}{2}(\bar{\partial}-\partial) \varphi$. Then $d \theta=i \partial \bar{\partial} \varphi$. Let us differentiate $\log |\varphi|=$ $-(1 /(n+1)) \log K$ (where $K$ is short for $K(z, z))$ so that to obtain

$$
\frac{1}{\varphi} \bar{\partial} \varphi=-\frac{1}{n+1} \bar{\partial} \log K
$$

Applying the operator $i \partial$ leads to

$$
\frac{1}{\varphi} d \theta-\frac{i}{\varphi^{2}} \partial \varphi \wedge \bar{\partial} \varphi=-\frac{i}{n+1} \partial \bar{\partial} \log K
$$

We shall need the Bergman metric

$$
g=\frac{\partial^{2} \log K}{\partial z^{j} \partial \bar{z}^{k}} d z^{j} \odot d \bar{z}^{k} .
$$

As well known, $g$ is a Kähler metric on $\Omega$ (Kähler-Einstein when $\Omega$ is homogeneous). Here $\odot$ denotes the symmetric tensor product, i.e. $\alpha \odot \beta=\frac{1}{2}(\alpha \otimes \beta+\beta \otimes \alpha)$. Let us set $\omega(X, Y)=g(X, J Y)$ (the Kähler 2 -form of $(\Omega, J, g)$, where $J$ is the underlying complex structure). Then $\omega=-i \partial \bar{\partial} \log K$ and (34) may be written

$$
g(X, Y)=\frac{n+1}{\varphi}\left\{\frac{i}{\varphi}(\partial \varphi \wedge \bar{\partial} \varphi)(X, J Y)-d \theta(X, J Y)\right\}
$$

for any $X, Y \in \mathcal{X}(\Omega)$.

We denote by $M_{\delta}=\{z \in \Omega: \varphi(z)=-\delta\}(\delta>0)$ the level sets of $\varphi$. For $\delta$ sufficiently small $M_{\delta}$ is still a strictly pseudoconvex CR manifold (of CR dimension $n-1$ ). Therefore, there is a one-sided neighborhood $V$ of $\partial \Omega$ which is foliated by the (strictly pseudoconvex) level sets of $\varphi$. Let $\mathcal{F}$ be the relevant foliation and let us denote by $H(\mathcal{F}) \rightarrow V$ (respectively by $T_{1,0}(\mathcal{F}) \rightarrow V$ ) the bundle whose portion over $M_{\delta}$ is the Levi distribution $H\left(M_{\delta}\right)$ (respectively the CR structure $T_{1,0}\left(M_{\delta}\right)$ ). Note that

$$
\begin{gathered}
T_{1,0}(\mathcal{F}) \cap T_{0,1}(\mathcal{F})=(0), \\
{\left[\Gamma^{\infty}\left(T_{1,0}(\mathcal{F})\right), \Gamma^{\infty}\left(T_{1,0}(\mathcal{F})\right)\right] \subseteq \Gamma^{\infty}\left(T_{1,0}(\mathcal{F})\right) .}
\end{gathered}
$$

Here $T_{0,1}(\mathcal{F})=\overline{T_{1,0}(\mathcal{F})}$. By a result in [20, there is a unique complex vector field $\xi$ on $V$, of type $(1,0)$, such that $\partial \varphi(\xi)=1$ and $\xi$ is orthogonal to $T_{1,0}(\mathcal{F})$ with respect to $\partial \bar{\partial} \varphi$, i.e. $\partial \bar{\partial} \varphi(\xi, \bar{Z})=0$, for any 
$Z \in T_{1,0}(\mathcal{F})$. We set $r \equiv 2 \partial \bar{\partial} \varphi(\xi, \bar{\xi})$ ( $r$ is the transverse curvature of $\varphi)$. Let $\xi=\frac{1}{2}(N-i T)$ be the real and imaginary parts of $\xi$. Then

$$
\begin{aligned}
(d \varphi)(N) & =2, \quad(d \varphi)(T)=0, \\
\theta(N) & =0, \quad \theta(T)=1, \\
\partial \varphi(N) & =1, \quad \partial \varphi(T)=i .
\end{aligned}
$$

In particular, $T$ is tangent to (the leaves of) $\mathcal{F} . \mathcal{F}$ carries the tangential Riemannian metric $g_{\theta}$ (defined by (100) in Appendix A). Note that the pullback of $g_{\theta}$ to each leaf $M_{\delta}$ of $\mathcal{F}$ is the Webster metric of $M_{\delta}$ (associated to the contact form $j_{\delta}^{*} \theta$, where $j_{\delta}: M_{\delta} \subset V$ ). As a consequence of (35)

$$
g(X, Y)=-\frac{n+1}{\varphi} g_{\theta}(X, Y), \quad X, Y \in H(\mathcal{F}) .
$$

Also (by $J T=-N$ and (103))

$$
\begin{gathered}
g(X, T)=0, \quad g(X, N)=0, \quad X \in H(\mathcal{F}), \\
g(T, N)=0, \quad g(T, T)=g(N, N)=\frac{n+1}{\varphi}\left(\frac{1}{\varphi}-r\right) .
\end{gathered}
$$

In particular $1-r \varphi>0$ everywhere in $\Omega$. Using (36) - (38) we may relate the Levi-Civita connection $\nabla^{g}$ of $(V, g)$ to the Graham-Lee connection $\nabla$ (cf. Appendix A). By (36) $($ as $X(\varphi)=0, X \in T(\mathcal{F}))$

$$
g\left(\nabla_{X}^{g} Y, Z\right)=g\left(\nabla_{X} Y, Z\right), \quad X, Y, Z \in H(\mathcal{F}) .
$$

Note that any tangent vector field $X \in T(V)$ decomposes as

$$
X=\pi_{H} X+\theta(X) T+\frac{1}{2}(d \varphi)(X) N,
$$

$\left(\pi_{H}: T(V) \rightarrow H(\mathcal{F})\right.$ is the projection). By (102) $\theta([T, X])=0, X \in$ $H(\mathcal{F})$. Also $[T, X] \in T(\mathcal{F})$, hence $[T, X] \in H(\mathcal{F})$, for any $X \in H(\mathcal{F})$. Taking into account the identity

$$
\begin{aligned}
& 2 g\left(\nabla_{X}^{g} Y, Z\right)=X(g(Y, Z))+Y(g(X, Z))-Z(g(X, Y))+ \\
& \quad+g([X, Y], Z)+g([Z, X], Y)+g(X,[Z, Y]),
\end{aligned}
$$

for any $X, Y, Z \in T(V)$, one has (by (37))

$$
\begin{gathered}
2 g\left(\nabla_{X}^{g} Y, T\right)=-T(g(X, Y))+ \\
+g([X, Y], T)+g([T, X], Y)+g(X,[T, Y])= \\
=\frac{n+1}{\varphi}\left\{T\left(g_{\theta}(X, Y)\right)-g_{\theta}([T, X], Y)-g_{\theta}(X,[T, Y])\right\}+
\end{gathered}
$$




$$
+\frac{n+1}{\varphi}\left(\frac{1}{\varphi}-r\right) \theta([X, Y]),
$$

for any $X, Y \in H(\mathcal{F})$. By (112)-(113) and $\nabla_{X} Y \in H(\mathcal{F})$ it follows that

$$
T\left(g_{\theta}(X, Y)\right)-g_{\theta}([T, X], Y)-g_{\theta}(X,[T, Y])=2 g_{\theta}(\tau X, Y)
$$

(note that one makes use of the fact that $\tau: H(\mathcal{F}) \rightarrow H(\mathcal{F})$ is selfadjoint, i.e. $\left.g_{\theta}(\tau X, Y)=g_{\theta}(X, \tau Y), X, Y \in H(\mathcal{F})\right)$ hence

$$
g\left(\nabla_{X}^{g} Y, T\right)=-g(\tau X, Y)-\frac{n+1}{\varphi}\left(\frac{1}{\varphi}-r\right)(d \theta)(X, Y)
$$

or

$$
g\left(\nabla_{X}^{g} Y, T\right)=-g(\tau X, Y)-\left(\frac{1}{\varphi}-r\right) g(X, \phi Y),
$$

for any $X, Y \in H(\mathcal{F})$. Exploiting again $\nabla^{g} g=0$ (and $g([X, Y], N)=$ 0 ) we get

$$
2 g\left(\nabla_{X}^{g} Y, N\right)=-\left(\mathcal{L}_{N} g\right)(X, Y), \quad X, Y \in H(\mathcal{F}) .
$$

Hence (by (122) in Lemma 4)

$$
\begin{gathered}
2 g\left(\nabla_{X}^{g} Y, N\right)=-\frac{n+1}{\varphi^{2}} N(\varphi) g_{\theta}(X, Y)+\frac{n+1}{\varphi}\left(\mathcal{L}_{N} g_{\theta}\right)(X, Y)= \\
=2\left(\frac{1}{\varphi}-r\right) g(X, Y)+\frac{2(n+1)}{\varphi}(d \theta)(X, \tau Y)
\end{gathered}
$$

that is

$$
g\left(\nabla_{X}^{g} Y, N\right)=\left(\frac{1}{\varphi}-r\right) g(X, Y)+g(X, \phi \tau Y),
$$

for any $X, Y \in H(\mathcal{F})$. Note that (42) may be also derived from (41) by using the fact that $g$ is a Kähler metric. Indeed

$$
\begin{gathered}
g\left(\nabla_{X}^{g} Y, N\right)=g\left(J \nabla_{X}^{g} Y, J N\right)=g\left(\nabla_{X}^{g} J Y, T\right)= \\
=-g(\tau X, \phi Y)-\left(\frac{1}{\varphi}-r\right) g\left(X, \phi^{2} Y\right),
\end{gathered}
$$

etc. For further use, let us also retain that

$$
\left(\mathcal{L}_{N} g\right)(X, Y)=-2\left(\frac{1}{\varphi}-r\right) g(X, Y)-2 g(X, \phi \tau Y),
$$

for any $X, Y \in H(\mathcal{F})$. At this point, the identities (39) and (41)-(42) lead to

$$
\nabla_{X}^{g} Y=\nabla_{X} Y+
$$




$$
\begin{aligned}
& +\left\{\frac{\varphi}{1-\varphi r} g_{\theta}(\tau X, Y)+g_{\theta}(X, \phi Y)\right\} T- \\
& -\left\{g_{\theta}(X, Y)+\frac{\varphi}{1-\varphi r} g_{\theta}(X, \phi \tau Y)\right\} N,
\end{aligned}
$$

for any $X, Y \in H(\mathcal{F})$. To compute $\nabla_{X}^{g} T$ we use (44) and

$$
g\left(\nabla_{X}^{g} T, Y\right)=-g\left(T, \nabla_{X}^{g} Y\right)
$$

so that

$$
g\left(\nabla_{X}^{g} T, Y\right)=g(\tau X, Y)+\left(\frac{1}{\varphi}-r\right) g(X, \phi Y) .
$$

The component along $T$ is $\frac{1}{2} X\left(\|T\|^{2}\right)$ hence

$$
g\left(\nabla_{X}^{g} T, T\right)=-\frac{n+1}{2 \varphi} X(r) .
$$

Moreover (by (104) in Appendix A)

$$
2 g\left(\nabla_{X}^{g} T, N\right)=g(X,[N, T])=-g\left(X, \phi \nabla^{H} r\right)
$$

that is

$$
g\left(\nabla_{X}^{g} T, N\right)=-\frac{n+1}{2 \varphi}(\phi X)(r) .
$$

Summing up (by (45)-(47))

(48) $\nabla_{X}^{g} T=\tau X-\left(\frac{1}{\varphi}-r\right) \phi X-\frac{\varphi}{2(1-r \varphi)}\{X(r) T+(\phi X)(r) N\}$,

for any $X \in H(\mathcal{F})$. Again by (44) and

$$
g\left(\nabla_{X}^{g} N, Y\right)=-g\left(N, \nabla_{X}^{g} Y\right)
$$

we get

$$
g\left(\nabla_{X}^{g} N, Y\right)=-\left(\frac{1}{\varphi}-r\right) g(X, Y)-g(X, \phi \tau Y) .
$$

Next (by (104))

$$
g\left(\nabla_{X}^{g} N, T\right)=\frac{n+1}{2 \varphi}(\phi X)(r) .
$$

Finally, the component along $N$ is $\frac{1}{2} X\left(\|N\|^{2}\right)$ hence

$$
g\left(\nabla_{X}^{g} N, N\right)=-\frac{n+1}{2 \varphi} X(r)
$$

Summing up (by (49)-(51))

$$
\nabla_{X}^{g} N=-\left(\frac{1}{\varphi}-r\right) X+\tau \phi X+
$$




$$
+\frac{\varphi}{2(1-r \varphi)}\{(\phi X)(r) T-X(r) N\}
$$

for any $X \in H(\mathcal{F})$. We wish to compute $\nabla_{T}^{g} X$. To this end (by $\left.\nabla g_{\theta}=0\right)$

$$
\begin{gathered}
2 g_{\theta}\left(\nabla_{T} X, Y\right)=T\left(g_{\theta}(X, Y)\right)+g_{\theta}([T, X], Y)+g_{\theta}([Y, T], X)+ \\
+g_{\theta}(T,[Y, X])+g_{\theta}(\tau X, Y)-g_{\theta}(\tau Y, X)-2(d \theta)(X, Y)
\end{gathered}
$$

yielding (upon multiplication by $-(n+1) / \varphi$ )

$$
T(g(X, Y))+g([T, X], Y)+g([Y, T], X)=2 g\left(\nabla_{T} X, Y\right) .
$$

Therefore (by $\nabla^{g} g=0$ )

$$
\begin{gathered}
2 g\left(\nabla_{T}^{g} X, Y\right)=T(g(X, Y))+g([T, X], Y)+g([Y, T], X)+ \\
+g(T,[Y, X])=2 g\left(\nabla_{T} X, Y\right)-\theta([X, Y])\|T\|^{2}
\end{gathered}
$$

or

$$
g\left(\nabla_{T}^{g} X, Y\right)=g\left(\nabla_{T} X, Y\right)+\left(\frac{1}{\varphi}-r\right) g(X, \phi Y) .
$$

Similar to the above

$$
\begin{gathered}
g\left(\nabla_{T}^{g} X, T\right)=-\frac{n+1}{2 \varphi} X(r), \\
g\left(\nabla_{T}^{g} X, N\right)=-\frac{n+1}{2 \varphi}(\phi X)(r) .
\end{gathered}
$$

Collecting the information in (53)-(55), we have proved

$$
\nabla_{T}^{g} X=\nabla_{T} X-\left(\frac{1}{\varphi}-r\right) \phi X-\frac{\varphi}{2(1-r \varphi)}\{X(r) T+(\phi X)(r) N\}
$$

for any $X \in H(\mathcal{F})$. Let us compute $\nabla_{N}^{g} X$. We have

$$
\begin{aligned}
2 g\left(\nabla_{N}^{g} X, Y\right) & =N(g(X, Y))+g([N, X], Y)+g([Y, N], X)= \\
& =2 g([N, X], Y)+\left(\mathcal{L}_{N} g\right)(X, Y) .
\end{aligned}
$$

Using (43) and

$$
[N, X]=\nabla_{N} X-r X-\tau(\phi X)
$$

(cf. Appendix A) one shows that

$$
g\left(\nabla_{N}^{g} X, Y\right)=g\left(\nabla_{N} X, Y\right)-\frac{1}{\varphi} g(X, Y) .
$$

Calculations similar to the above also furnish

$$
g\left(\nabla_{N}^{g} X, T\right)=\frac{n+1}{2 \varphi}(\phi X)(r)
$$




$$
g\left(\nabla_{N}^{g} X, N\right)=-\frac{n+1}{2 \varphi} X(r)
$$

Using (57)-(59) we may now conclude that

$$
\nabla_{N}^{g} X=\nabla_{N} X-\frac{1}{\varphi} X+\frac{\varphi}{2(1-r \varphi)}\{(\phi X)(r) T-X(r) N\},
$$

for any $X \in H(\mathcal{F})$. Moreover (omitting the details)

so that

$$
\begin{gathered}
g\left(\nabla_{N}^{g} T, X\right)=-\frac{n+1}{2 \varphi}(\phi X)(r), \\
g\left(\nabla_{N}^{g} T, T\right)=-\frac{n+1}{2 \varphi}\left\{N(r)+\frac{4}{\varphi^{2}}-\frac{2 r}{\varphi}\right\}, \\
g\left(\nabla_{N}^{g} T, N\right)=-\frac{n+1}{2 \varphi} T(r),
\end{gathered}
$$

$$
\begin{gathered}
\nabla_{N}^{g} T=-\frac{1}{2} \phi \nabla^{H} r- \\
-\frac{\varphi}{2(1-r \varphi)}\left\{\left(N(r)+\frac{4}{\varphi^{2}}-\frac{2 r}{\varphi}\right) T+T(r) N\right\} .
\end{gathered}
$$

Similarly we find

$$
\begin{gathered}
\nabla_{T}^{g} N=\frac{1}{2} \phi \nabla^{H} r- \\
-\frac{\varphi}{2(1-r \varphi)}\left\{\left(N(r)+\frac{4}{\varphi^{2}}-\frac{6 r}{\varphi}+4 r^{2}\right) T+T(r) N\right\}, \\
\nabla_{T}^{g} T=-\frac{1}{2} \nabla^{H} r- \\
-\frac{\varphi}{2(1-r \varphi)}\left\{T(r) T-\left(N(r)+\frac{4}{\varphi^{2}}-\frac{6 r}{\varphi}+4 r^{2}\right) N\right\}, \\
+\frac{\varphi}{2(1-r \varphi)}\left\{T(r) T-\left(N(r)+\frac{4}{\varphi^{2}}-\frac{2 r}{\varphi}\right) N\right\} .
\end{gathered}
$$

Let us consider a holomorphic vector bundle $\pi: F \rightarrow U$, carrying the Hermitian metric $h$, and set $E_{\delta}=\pi^{-1}\left(M_{\delta}\right)$ (the portion of $F$ over a leaf of $\mathcal{F})$. A connection $D \in \mathcal{C}(F, h)$ induces a connection $D^{\delta} \in \mathcal{C}\left(E_{\delta}, h_{\delta}\right)$ (where $h_{\delta, z}=h_{z}, z \in M_{\delta}$ ). $D^{\delta}$ is most easily described with respect to a local trivialization $\Phi: \pi^{-1}(O) \rightarrow O \times \mathbb{C}^{m}$ of $F$, for some open 
subset $O \subseteq U$. Let us set $\sigma_{i}(z)=\Phi^{-1}\left(z, e_{i}\right), z \in O, 1 \leq i \leq m$, where $\left\{e_{1}, \cdots, e_{m}\right\}$ is the canonical linear basis in $\mathbb{C}^{m}$. If $\left.u_{i} \equiv \sigma_{i}\right|_{O \cap M_{\delta}}$ then $D^{\delta}$ is given by

$$
\left(D_{X}^{\delta} u\right)_{z}=X\left(f^{i}\right)_{z} u_{i}(z)+f^{i}(z)\left(D_{\left(d j_{\delta}\right) X} \sigma_{i}\right)_{z}, \quad z \in O \cap M_{\delta},
$$

for any section $u=f^{i} u_{i}, f^{i} \in C^{\infty}\left(O \cap M_{\delta}\right)$, and any $X \in \mathcal{X}\left(M_{\delta}\right)$. It is easily shown that the definition of $\left(D_{X}^{\delta} u\right)_{z}$ doesn't depend upon the local trivialization chart $\Phi$ at $z$ (i.e. if $g=\left[g_{i j}\right]: O \cap O^{\prime} \rightarrow$ $\mathrm{GL}(m, \mathbb{C}), g(z)=\Phi_{z}^{\prime} \circ \Phi_{z}^{-1}$, are the transition functions of $F$ then $\left(D_{X}^{\delta} u\right)_{z}$ is invariant under the transformation $\left.\sigma_{j}(z)=g_{j}^{i}(z) \sigma_{i}^{\prime}(z)\right)$. Let $R^{D} \in \Omega^{2}(\operatorname{Ad}(F))$ and $\omega_{j}^{i}$ be the curvature tensor field and connection 1-forms of $D\left(D \sigma_{j}=\omega_{j}^{i} \otimes \sigma_{i}\right)$, so that $R^{D} \sigma_{j}=2\left(d \omega_{j}^{i}-\omega_{k}^{i} \wedge \omega_{j}^{k}\right) \otimes \sigma_{i}$. Also, let $R^{\delta} \in \Omega^{2}\left(\operatorname{Ad}\left(E_{\delta}\right)\right)$ and $\left(\omega_{\delta}\right)_{j}^{i}$ be the curvature tensor field and the connection 1-forms of $D^{\delta}$, respectively. Then $\left(\omega_{\delta}\right)_{j}^{i}=j_{\delta}^{*} \omega_{j}^{i}$ yields

$$
R^{\delta} u_{i}=\left(j_{\delta}^{*} R^{D}\right) \sigma_{i}, \quad 1 \leq i \leq m .
$$

Let $\left\{W_{\alpha}\right\}$ be a local orthonormal $\left(g_{\theta}\left(W_{\alpha}, W_{\bar{\beta}}\right)=\delta_{\alpha \beta}\right)$ frame of $T_{1,0}(\mathcal{F})$ and set

$$
E_{\alpha} \equiv \sqrt{-\frac{\varphi}{n+1}} W_{\alpha}, \quad 1 \leq \alpha \leq n-1, \quad E_{n} \equiv \sqrt{\frac{2 f \varphi}{n+1}} \xi,
$$

where $f \equiv \varphi /(1-r \varphi)$. Then, given a connection $D$ in $F \rightarrow U$, for any $X \in H\left(M_{\delta}\right)$

$$
\begin{gathered}
\left(\delta^{D} R^{D}\right) X=-\sum_{a=1}^{n}\left\{\left(D_{E_{a}} R^{D}\right)\left(E_{\bar{a}}, X\right)+\left(D_{E_{\bar{a}}} R^{D}\right)\left(E_{a}, X\right)\right\}= \\
=\frac{\varphi}{n+1} \sum_{\alpha=1}^{n-1}\left\{\left(D_{W_{\alpha}} R^{D}\right)\left(W_{\bar{\alpha}}, X\right)+\left(D_{W_{\bar{\alpha}}} R^{D}\right)\left(W_{\alpha}, X\right)\right\}- \\
-\frac{2 f \varphi}{n+1}\left\{\left(D_{\xi} R^{D}\right)(\bar{\xi}, X)+\left(D_{\bar{\xi}} R^{D}\right)(\xi, X)\right\}
\end{gathered}
$$

and

$$
\begin{gathered}
\sum_{\alpha=1}^{n-1}\left(D_{W_{\alpha}} R^{D}\right)\left(W_{\bar{\alpha}}, X\right) \sigma_{j}= \\
=\sum_{\alpha}\left\{D_{W_{\alpha}}\left(R^{D}\left(W_{\bar{\alpha}}, X\right) \sigma_{j}\right)-R^{D}\left(W_{\bar{\alpha}}, X\right) D_{W_{\alpha}} \sigma_{j}-\right. \\
\left.-R^{D}\left(\nabla_{W_{\alpha}}^{g} W_{\bar{\alpha}}, X\right) \sigma_{j}-R^{D}\left(W_{\bar{\alpha}}, \nabla_{W_{\alpha}}^{g} X\right) \sigma_{j}\right\}=(b y \text { (444) }) \\
=\sum_{\alpha}\left\{\left(D_{W_{\alpha}}^{\delta} R^{\delta}\right)\left(W_{\bar{\alpha}}, X\right) u_{j}-\right. \\
-\left[f g_{\theta}\left(\tau W_{\alpha}, W_{\bar{\alpha}}\right)+g_{\theta}\left(W_{\alpha}, \phi W_{\bar{\alpha}}\right)\right] R^{D}(T, X) \sigma_{j}+
\end{gathered}
$$




$$
\begin{aligned}
& +\left[g_{\theta}\left(W_{\alpha}, W_{\bar{\alpha}}\right)+f g_{\theta}\left(W_{\alpha}, \phi \tau W_{\bar{\alpha}}\right)\right] R^{D}(N, X) \sigma_{j}- \\
& -\left[f g_{\theta}\left(\tau W_{\alpha}, X\right)+g_{\theta}\left(W_{\alpha}, \phi X\right)\right] R^{D}\left(W_{\bar{\alpha}}, T\right) \sigma_{j}+ \\
& \left.+\left[g_{\theta}\left(W_{\alpha}, X\right)+f g_{\theta}\left(W_{\alpha}, \phi \tau X\right)\right] R^{D}\left(W_{\bar{\alpha}}, N\right) \sigma_{j}\right\} .
\end{aligned}
$$

Therefore (by the purity axiom (107))

$$
\begin{gathered}
\sum_{\alpha}\left(D_{W_{\alpha}} R^{D}\right)\left(W_{\bar{\alpha}}, X\right)=\sum_{\alpha}\left(D_{W_{\alpha}}^{\delta} R^{\delta}\right)\left(W_{\bar{\alpha}}, X\right) u_{j}- \\
+i(n-1) R^{D}(T, X) \sigma_{j}+(n-1) R^{D}(N, X) \sigma_{j}- \\
\quad-f R^{D}\left(\pi_{0,1} \tau X, T\right)-R^{D}\left(\pi_{0,1} \phi X, T\right)+ \\
\quad+R^{D}\left(\pi_{0,1} X, N\right)+f R^{D}\left(\pi_{0,1} \phi \tau X, N\right) .
\end{gathered}
$$

We obtain

$$
\begin{aligned}
& \sum_{\alpha}\left\{\left(D_{W_{\alpha}} R^{D}\right)\left(W_{\bar{\alpha}}, X\right)+\left(D_{W_{\bar{\alpha}}} R^{D}\right)\left(W_{\alpha}, X\right)\right\}=-\left(\delta_{b}^{D^{\delta}} R^{\delta}\right)_{X} u_{j}+ \\
& +\left\{R^{D}(N,(2 n-3) X-f \phi \tau X)+R^{D}(T, \phi X+f \tau X)\right\} \sigma_{j}
\end{aligned}
$$

(cf. section 5 for the definition of the operator $\delta_{b}^{D^{\delta}}$ ). Moreover

$$
\begin{gathered}
\left\{\left(D_{\xi} R^{D}\right)(\bar{\xi}, X)+\left(D_{\bar{\xi}} R^{D}\right)(\xi, X)\right\} \sigma_{j}= \\
=\frac{1}{2}\left\{D_{N}\left(R^{D}(N, X) \sigma_{j}\right)+D_{T}\left(R^{D}(T, X) \sigma_{j}\right)-\right. \\
-R^{D}(N, X) D_{N} \sigma_{j}-R^{D}(T, X) D_{T} \sigma_{j}- \\
-R^{D}\left(\nabla_{N}^{g} N, X\right) \sigma_{j}-R^{D}\left(\nabla_{T}^{g} T, X\right) \sigma_{j}- \\
\left.-R^{D}\left(N, \nabla_{N}^{g} X\right) \sigma_{j}-R^{D}\left(T, \nabla_{T}^{g} X\right) \sigma_{j}\right\} .
\end{gathered}
$$

Substitution from (56), (60) and (63)-(64) gives

$$
\begin{gathered}
-R^{D}\left(\nabla_{N}^{g} N, X\right)-R^{D}\left(\nabla_{T}^{g} T, X\right)-R^{D}\left(N, \nabla_{N}^{g} X\right)-R^{D}\left(T, \nabla_{T}^{g} X\right)= \\
=R^{D}\left(\nabla^{H} r, X\right)-R^{D}\left(T, \nabla_{T} X\right)-R^{D}\left(N, \nabla_{N} X\right)+ \\
+\frac{1}{f} R^{D}(T, \phi X)+f(\phi X)(r) R^{D}(T, N)+\left(\frac{1}{\varphi}+2 r\right) R^{D}(N, X) .
\end{gathered}
$$

We conclude that

$$
\begin{gathered}
\left\{\left(D_{\xi} R^{D}\right)(\bar{\xi}, X)+\left(D_{\bar{\xi}} R^{D}\right)(\xi, X)\right\} \sigma_{j}= \\
=\frac{1}{2}\left\{\left(D_{N} i_{N} R^{D}\right) X+\left(D_{T} i_{T} R^{D}\right) X+R^{D}\left(\nabla^{H} r, X\right)+\right. \\
+\frac{1}{f} R^{D}(T, \phi X)+f(\phi X)(r) R^{D}(T, N)+\left(\frac{1}{\varphi}+2 r\right) R^{D}(N, X)
\end{gathered}
$$

(the covariant derivatives in the right hand member of (67) are defined with respect to $D$ and $\nabla$ ). Finally (by (66)-(67))

$$
\left(\delta^{D} R^{D}\right)_{X} \sigma_{j}=\frac{\varphi}{n+1}\left\{-\left(\delta_{b}^{D^{\delta}} R^{\delta}\right)_{X} u_{j}+\right.
$$




$$
\begin{aligned}
+ & {\left.\left[R^{D}(N,(2 n-3) X-f \phi \tau X)+R^{D}(T, \phi X+f \tau X)\right] \sigma_{j}\right\}- } \\
& -\frac{f \varphi}{n+1}\left\{\left(D_{N} i_{N} R^{D}\right) X+\left(D_{T} i_{T} R^{D}\right) X+R^{D}\left(\nabla^{H} r, X\right)+\right. \\
+ & \left.R^{D}\left(T, \frac{1}{f} \phi X+f(\phi X)(r) N\right)+\left(\frac{1}{\varphi}+2 r\right) R^{D}(N, X)\right\} \sigma_{j} .
\end{aligned}
$$

Assume that $D$ is a Yang-Mills field on $(\Omega, g)$, i.e. $\delta^{D} R^{D}=0$ in $\Omega$. Then, for $\varphi \rightarrow 0$ (as $r$ and $\nabla^{H} r$ stay finite near $\partial \Omega$, cf. [1], p. 164)

$$
\left(\delta_{b}^{D_{b}} R^{D_{b}}\right)_{X} u_{j}=2(n-2) R^{D}(N, X) \sigma_{j}
$$

where $D_{b} \equiv D^{0}$ is the boundary values of $D$. Therefore, if $i_{T} R^{D_{b}}=0$ then (cf. (84) in section 5) $D_{b}$ is a pseudo Yang-Mills field on $\partial \Omega$ if and only if $i_{N} R^{D}=0$ on $H(\partial \Omega)$. Theorem 1 is proved. With the same techniques we may show that

Corollary 1. Let $D \in \mathcal{C}(F, h)$ be a Yang-Mills field on $(\Omega, g)$ such that $i_{N} R^{D}=0$. Then the boundary values $D_{b}$ of $D$ satisfy $\Lambda_{\theta} R^{D_{b}}=0$.

Corollary 11 shows that the axiom (17) (with $S=0$ ) in the description of the Tanaka connection, as well as (11) in Theorem 2, are rather natural occurrences. The proof is

$$
\begin{gathered}
0=\left(\delta^{D} R^{D}\right)_{T} \sigma_{j}= \\
=\frac{\varphi}{n+1} \sum_{\alpha}\left\{\left(D_{W_{\alpha}} R^{D}\right)\left(W_{\bar{\alpha}}, T\right)+\left(D_{W_{\bar{\alpha}}} R^{D}\right)\left(W_{\alpha}, T\right)\right\} \sigma_{j}- \\
-\frac{2 f \varphi}{n+1}\left\{D_{N}\left(R^{D}(N, T) \sigma_{j}\right)-R^{D}(N, T) D_{N} \sigma_{j}-\right. \\
\left.-R^{D}\left(\nabla_{N}^{g} N, T\right)-R^{D}\left(N, \nabla_{N}^{g} T\right) \sigma_{j}\right\}
\end{gathered}
$$

or (by (44), (48), (61) and (64))

$$
\begin{gathered}
0=\varphi\left\{\left(\delta^{D^{\delta}} R^{\delta}\right)_{T} u_{j}+2(n-1) R^{D}(T, N) \sigma_{j}\right\}+ \\
+\frac{1}{2} \varphi\left\{f\left[R^{D}\left(T, \nabla^{H} r\right)+R^{D}\left(N, \phi \nabla^{H} r\right)\right]+\operatorname{trace} \pi_{H} R^{D}(\cdot, \tau \cdot)\right\} \sigma_{j}- \\
+(n+1) f\left\{2\left(D_{N} i_{N} R^{D}\right) T+R^{D}\left(N, \phi \nabla^{H} r\right)-R^{D}\left(T, \nabla^{H} r\right)\right\} \sigma_{j}- \\
-\frac{2 \varphi}{f} \Lambda_{\theta} R^{D} \sigma_{j}-(n+1) f^{2}\left\{N(r)+\frac{4}{\varphi^{2}}-\frac{2 r}{\varphi}\right\} R^{D}(T, N) \sigma_{j} .
\end{gathered}
$$

When $\varphi \rightarrow 0$ one observes $\varphi / f \rightarrow 1$ and $f^{2} / \varphi^{2} \rightarrow 1$ hence

$$
\left(\Lambda_{\theta} R^{D_{b}}\right) u_{j}=-2(n+1) R^{D}(T, N) \sigma_{j} .
$$

Q.e.d. 


\section{YANG-Mills Fields AND the FefFERMAn Metric}

We wish to relate $\mathcal{P} \mathcal{Y} \mathcal{M}$ to the Yang-Mills functional on $C(M)$. Given a contact form $\theta$ on $M$ such that the Levi form $L_{\theta}$ is positive definite, let $F_{\theta}$ be the corresponding Fefferman metric (a Lorentz metric on $C(M)$ ). We recall (cf. [18]) that

$$
\begin{gathered}
F_{\theta}=\pi^{*} \tilde{G}_{\theta}+2\left(\pi^{*} \theta\right) \odot \sigma \\
\sigma=\frac{1}{n+2}\left\{d \gamma+\pi^{*}\left(i \omega_{\alpha}^{\alpha}-\frac{i}{2} g^{\alpha \bar{\beta}} d g_{\alpha \bar{\beta}}-\frac{\rho}{4(n+1)} \theta\right)\right\} .
\end{gathered}
$$

Here $\omega_{\beta}^{\alpha}$ are the connection 1-forms of the Tanaka-Webster connection of $(M, \theta)$, i.e. $\nabla T_{\beta}=\omega_{\beta}^{\alpha} \otimes T_{\alpha}$, and $g_{\alpha \bar{\beta}}=L_{\theta}\left(T_{\alpha}, T_{\bar{\beta}}\right)$. Moreover $\rho=g^{\alpha \bar{\beta}} R_{\alpha \bar{\beta}}$ is the pseudohermitian scalar curvature (cf. e.g. [5], p. 229). The $(0,2)$-tensor field $\tilde{G}_{\theta}$ is got by extending the Levi form $G_{\theta}$ to the whole of $T(M)$. Precisely, one requests that $\tilde{G}_{\theta}=G_{\theta}$ on $H(M) \otimes H(M)$, while $\tilde{G}_{\theta}(X, T)=0$, for any $X \in T(M)$ (obviously $\tilde{G}_{\theta}$ is degenerate). Note that when $M$ is compact $C(M)$ is compact, as well. It is noteworthy that $\sigma$ (given by (69)) is a connection 1 -form in $S^{1} \rightarrow C(M) \rightarrow M$. Let $T^{\uparrow}$ be the horizontal lift (with respect to $\sigma$ ) of the characteristic direction of $d \theta$ and $S$ the tangent to the $S^{1}$-action. Then $T^{\uparrow}-S$ is timelike, hence $\left(C(M), F_{\theta}\right)$ is time oriented by $T^{\uparrow}-S$, i.e. $\left(C(M), F_{\theta}\right)$ is a space-time (see [3, p. 17). However, as $M$ is compact $\left(C(M), F_{\theta}\right)$ is not chronological (cf. Proposition 2.6 in [3], p. 23).

Let $S \in \mathcal{X}(C(M))$ be the tangent to the $S^{1}$-action (locally $S=$ $((n+2) / 2) \partial / \partial \gamma)$. Then (by (68) $) F_{\theta}(S, S)=0$. Next (by Lemma 2) $\nabla_{S}^{C(M)} S=0$, i.e. the integral curves of $S$ are (null) geodesics of $\left(C(M), F_{\theta}\right)$. Also $\mathcal{L}_{S} F_{\theta}=0$, hence (cf. (28) in [26], p. 185) $S$ generates a shear-free congruence of null geodesics. The congruence is symmetric if there is a vector field $X \in \mathcal{X}(C(M))$ such that

$$
\mathcal{L}_{X}\left(\pi^{*} \theta\right)=t \pi^{*} \theta, \quad \mathcal{L}_{X}\left(\pi^{*} \theta^{\alpha}\right)=w_{\beta}^{\alpha} \pi^{*} \theta^{\beta}+\ell^{\alpha} \pi^{*} \theta,
$$

where $t$ is a real function and $w_{\beta}^{\alpha}, \ell^{\alpha}$ are complex functions on $C(M)$. We say that $X$ is a symmetry of the congruence. We may look for $\mathcal{L}_{X} S$ in the form

$$
\mathcal{L}_{X} S=a^{\alpha} T_{\alpha}^{\uparrow}+a^{\bar{\alpha}} T_{\bar{\alpha}}^{\uparrow}+b T^{\uparrow}+f S .
$$

As $X$ is a symmetry $a^{\alpha}=0, b=0$ and $f=-2\left(\mathcal{L}_{X} \sigma\right) S$. Therefore

$$
\mathcal{L}_{X} S=f S .
$$


Also one may easily check (by using the local frame $\left\{T_{\alpha}^{\uparrow}, T_{\bar{\alpha}}^{\uparrow}, T^{\uparrow}, S\right\}$ of $T(C(M)) \otimes \mathbb{C})$ that

$$
\mathcal{L}_{S}\left(\pi^{*} \theta\right)=0, \quad \mathcal{L}_{S}\left(\pi^{*} \theta^{\alpha}\right)=0 .
$$

Using (70)-(71) and $\mathcal{L}_{Y} \mathcal{L}_{Z} \omega=\mathcal{L}_{Z} \mathcal{L}_{Y} \omega+\mathcal{L}_{[Y, Z]} \omega$ (for any $Y, Z \in$ $\left.\mathcal{X}(C(M)), \omega \in \Omega^{1}(C(M))\right)$ we obtain

$$
S(t)=0, \quad S\left(w_{\beta}^{\alpha}\right)=0, \quad S\left(\ell^{\alpha}\right)=0 .
$$

For instance

$$
S(t) \pi^{*} \theta=\mathcal{L}_{[S, X]} \pi^{*} \theta=-f \mathcal{L}_{S} \pi^{*} \theta-\left(\pi^{*} \theta\right)(S) d f=0 .
$$

Our considerations draw inspiration from the calculations in 24] (which are both purely local and confined to the 3-dimensional case $(n=1))$. For this reason some of the results (e.g. Propositions 1] and 21) are attributed to [24] (the proofs are however new). (172) implies that $t, w_{\beta}^{\alpha}, \ell^{\alpha}$ are vertical lifts of functions on $M$. A vector field of the form $\rho S$, for some function $\rho \neq 0$, is a trivial symmetry of the congruence.

Proposition 2. (P. Nurowski, [24])

Each nontrivial symmetry of the shear-free congruence (of null geodesics on $C(M)$ ) projects on a unique symmetry of the $C R$ structure on $M$.

Indeed, if $X$ is a symmetry of $S$ then $X-2 \sigma(X) S \in \operatorname{Ker}(\sigma)$, hence there is a unique vector field $\tilde{X} \in \mathcal{X}(M)$ such that

$$
\tilde{X}^{\uparrow}=X-2 \sigma(X) S .
$$

Then

$$
\pi^{*}(t \theta)=\mathcal{L}_{X}\left(\pi^{*} \theta\right)=\mathcal{L}_{\tilde{X}^{\uparrow}}\left(\pi^{*} \theta\right)+2 \mathcal{L}_{\sigma(X) S}\left(\pi^{*} \theta\right)=\mathcal{L}_{\tilde{X}^{\uparrow}}\left(\pi^{*} \theta\right) .
$$

Consequently, for any $Z \in \mathcal{X}(M)$

$$
t \theta(Z)=\left(\pi^{*}(t \theta)\right) Z^{\uparrow}=\left(\mathcal{L}_{\tilde{X}^{\uparrow}}\left(\pi^{*} \theta\right)\right) Z^{\uparrow}=\tilde{X}(\theta(Z))-\left(\pi^{*} \theta\right)\left[\tilde{X}^{\uparrow}, Z^{\uparrow}\right]
$$

hence, as $[\tilde{X}, Z]^{\uparrow}$ is the $\operatorname{Ker}(\sigma)$-component of $\left[\tilde{X}^{\uparrow}, Z^{\uparrow}\right]$ (with respect to the decomposition $T(C(M))=\operatorname{Ker}(\sigma) \oplus \mathbb{R} S)$, we obtain $\mathcal{L}_{\tilde{X}} \theta=t \theta$. It may be shown in a similar manner that $\mathcal{L}_{\tilde{X}} \theta^{\alpha}=w_{\beta}^{\alpha} \theta^{\beta}+\ell^{\alpha} \theta$, i.e. $\tilde{X}$ is a symmetry of the CR structure. Q.e.d.

Let $E \rightarrow M$ be a complex vector bundle and $\hat{E}=\pi^{*} E \rightarrow C(M)$ the pullback of $E$ via $\pi$. The natural lift $\hat{u}: \pi^{-1}(U) \rightarrow \hat{E}$ of a section $u: U \rightarrow E$ is given by $\hat{u}(z)=(x, u(\pi(z))), z \in \pi^{-1}(U)$. If $E$ carries a Hermitian metric $h$ then so does $\hat{E}$. Indeed we may set $\hat{h}\left(\hat{e}_{i}, \hat{e}_{j}\right)=$ $h_{i \bar{j}} \circ \pi$, where $h_{i \bar{j}}=h\left(e_{i}, e_{j}\right)$ and $\left\{e_{1}, \cdots, e_{m}\right\}$ is a (local) frame in $E$ 
on $U$. There is a natural inner product $\langle$,$\rangle on \Omega^{2}(\operatorname{Ad}(\hat{E}))$ induced by the inner product on scalar 2-forms

$$
F_{\theta}^{*}(\alpha, \beta) d \operatorname{vol}\left(F_{\theta}\right)=\alpha \wedge * \beta,
$$

$\alpha, \beta \in \Gamma^{\infty}\left(\Lambda^{2} T^{*}(C(M))\right)$, and by the Killing-Cartan form of $\mathbf{u}(m)$, $m=\operatorname{rank}_{\mathbb{C}} E$, respectively. Here $*$ is the Hodge operator associated with the Fefferman metric $F_{\theta}$. Precisely, if $S, T \in \Omega^{0}(\operatorname{Ad}(\hat{E}))$ then

$$
\langle\alpha \otimes S, \beta \otimes T\rangle=F_{\theta}^{*}(\alpha, \beta)\left[S_{j}^{i}\right] \cdot\left[T_{j}^{i}\right],
$$

where $S \hat{e}_{j}=S_{j}^{i} \hat{e}_{i}, T \hat{e}_{j}=T_{j}^{i} \hat{e}_{i}$ with respect to a (local) orthonormal $\left(h\left(e_{i}, e_{j}\right)=\delta_{i j}\right)$ frame $\left\{e_{j}\right\}$ in $E$, and $A \cdot B=-\operatorname{trace}(A B), A, B \in \mathbf{u}(m)$. The Yang-Mills functional is given by

$$
\widehat{\mathcal{Y M}}(\mathbb{D})=\frac{1}{2} \int_{C(M)}\left\langle R^{\mathbb{D}}, R^{\mathbb{D}}\right\rangle d \operatorname{vol}\left(F_{\theta}\right), \quad \mathbb{D} \in \mathcal{C}(\hat{E}, \hat{h}) .
$$

Any $D \in \mathcal{C}(E, h)$ induces a connection $\hat{D}=\pi^{*} D \in \mathcal{C}(\hat{E}, \hat{h})$ which is described (in local coordinates) as follows. Let $\left(U, x^{A}\right)$ be a local coordinate system on $M$. Then $\left(\pi^{-1}(U), \hat{x}^{A}:=x^{A} \circ \pi, \gamma\right)$ are local coordinates on $C(M)$. We set by definition

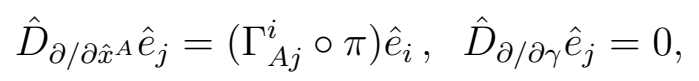

where $D_{\partial / \partial x^{A}} e_{j}=\Gamma_{A j}^{i} e_{i}$. Our conventions as to the range of indices are $A, B, C, \cdots \in\{1, \cdots, 2 n+1\}$ and $i, j, k, \cdots \in\{1, \cdots, m\}$. We consider the linear map

$$
\pi^{*}: \Gamma^{\infty}\left(U, \Lambda^{k} T^{*}(M) \otimes E\right) \rightarrow \Gamma^{\infty}\left(\pi^{-1}(U), \Lambda^{k} T^{*}(C(M)) \otimes \hat{E}\right)
$$

given by

$$
\pi^{*}\left(\omega^{j} \otimes e_{j}\right)=\left(\pi^{*} \omega^{j}\right) \otimes \hat{e}_{j}, \quad \omega^{j} \in \Omega^{k}(U),
$$

(pullback and natural lifting). As $\left\{\hat{e}_{j}\right\}$ is a local frame in $\hat{E} \rightarrow C(M)$ it suffices to specify $\hat{D}$ on natural lifts of sections in $E \rightarrow M$. Then $\hat{D}$ admits the following coordinate-free description

$$
\hat{D} \hat{u}=\pi^{*}(D u), \quad u \in \Omega^{0}(E) .
$$

Clearly, if $D h=0$ then $\hat{D} \hat{h}=0$. Let us consider the functional $\mathcal{P} \mathcal{Y} \mathcal{M}$ : $\mathcal{C}(E, h) \rightarrow[0,+\infty)$ given by

$$
\mathcal{P Y \mathcal { M }}(D)=\frac{1}{2} \int_{M}\left\|\pi_{H} R^{D}\right\|^{2} \theta \wedge(d \theta)^{n} .
$$

Here $\pi_{H}: \Omega^{2}(\operatorname{Ad}(E)) \rightarrow \Omega^{2}(\operatorname{Ad}(E)) / \mathcal{J}_{\theta}^{2}$ is the projection described in section 2. Of course, when an admissible coframe $\left\{\theta^{\alpha}\right\}$ is fixed $\Omega(\operatorname{Ad}(E)) / \mathcal{J}_{\dot{\theta}}$ may be identified with the subalgebra $\Omega_{H}(\operatorname{Ad}(E))=$ 
$\left\{\omega \in \Omega(\operatorname{Ad}(E)): i_{T} \omega=0\right\}$. Integration along the fibre in $\widehat{\mathcal{Y M}}\left(\pi^{*} D\right)$, $D \in \mathcal{C}(E, h)$, leads to (9) in Theorem 2. Indeed, let us set

$$
R_{A B j}^{i} e_{i}=\left(R^{D} e_{j}\right)\left(\partial / \partial x^{A}, \partial / \partial x^{B}\right) .
$$

Then

$$
R^{\hat{D}} \hat{e}_{j}=\left[\left(R_{A B j}^{i} \circ \pi\right) d \hat{x}^{A} \wedge d \hat{x}^{B}\right] \otimes \hat{e}_{i}
$$

hence

$$
R^{\hat{D}} \hat{e}_{j}=\pi^{*}\left(R^{D} e_{j}\right)
$$

Given $\Omega=\Omega^{j} \otimes e_{j} \in \Omega^{2}(E), \Omega^{j}=\Omega_{A B}^{j} d x^{A} \wedge d x^{B}$, we set

$$
\left\langle\pi^{*} \Omega, \pi^{*} \Omega\right\rangle=F_{\theta}^{*}\left(\pi^{*} \Omega^{j}, \pi^{*} \Omega^{\bar{k}}\right)\left(h_{j \bar{k}} \circ \pi\right) .
$$

Of course $\pi^{*} \Omega^{j}=\left(\Omega_{A B}^{j} \circ \pi\right) d \hat{x}^{A} \wedge d \hat{x}^{B}$ and the main technical difficulty in calculating (174) is the need for $F^{A B}=F_{\theta}^{*}\left(d \hat{x}^{A}, d \hat{x}^{B}\right)$, where $\left[F^{A B}\right]=$ $\left[F_{A B}\right]^{-1}$ and $F_{A B}:=F_{\theta}\left(\partial / \partial \hat{x}^{A}, \partial / \partial \hat{x}^{B}\right)$. Let

$$
F_{\theta}:\left[\begin{array}{cc}
F_{A B} & F_{A, 2 n+2} \\
F_{2 n+2, B} & F_{2 n+2,2 n+2}
\end{array}\right]
$$

be the components of the Fefferman metric with respect to $\left(\hat{x}^{A}, \gamma\right)$. Let us set $\partial / \partial x^{A}=\lambda_{A}^{B} T_{B}, \lambda_{A}^{B} \in C^{\infty}(U)$. Here one either adopts the convention $A, B, C, \cdots \in\{0,1, \cdots, n, \overline{1}, \cdots, \bar{n}\}$ (with $T_{0}=T$ ) or relabels the vector fields $\left\{T, T_{\alpha}, T_{\bar{\alpha}}: 1 \leq \alpha \leq n\right\}$. Then (by (68))

$$
\begin{gathered}
F_{A B}=\tilde{G}_{\theta}\left(\frac{\partial}{\partial x^{A}}, \frac{\partial}{\partial x^{B}}\right)+\theta\left(\frac{\partial}{\partial x^{A}}\right) \sigma\left(\frac{\partial}{\partial \hat{x}^{B}}\right)+\theta\left(\frac{\partial}{\partial x^{B}}\right) \sigma\left(\frac{\partial}{\partial \hat{x}^{A}}\right)= \\
=g_{\alpha \bar{\beta}}\left(\lambda_{A}^{\alpha} \lambda_{B}^{\bar{\beta}}+\lambda_{B}^{\alpha} \lambda_{A}^{\bar{\beta}}\right)+\lambda_{A}^{0} \sigma_{B}+\lambda_{B}^{0} \sigma_{A}
\end{gathered}
$$

where $\sigma_{A}=\sigma\left(\partial / \partial \hat{x}^{A}\right)$. A calculation based on (69) shows that

$$
\sigma_{A}=\frac{1}{n+2}\left\{i \lambda_{A}^{B}\left(\Gamma_{B \alpha}^{\alpha}-\frac{1}{2} g^{\alpha \bar{\beta}} T_{B}\left(g_{\alpha \bar{\beta}}\right)\right)-\frac{\rho}{4(n+1)} \lambda_{A}^{0}\right\} \circ \pi
$$

where $\Gamma_{B \alpha}^{\beta}$ are (among) the coefficients of the Tanaka-Webster connection of $(M, \theta)$ (i.e. $\nabla_{T_{B}} T_{\alpha}=\Gamma_{B \alpha}^{\beta} T_{\beta}$ ). Moreover (by (68) $)$

$$
\begin{gathered}
F_{A, 2 n+2}=2\left[\left(\pi^{*} \theta\right) \odot \sigma\right]\left(\frac{\partial}{\partial \hat{x}^{A}}, \frac{\partial}{\partial \gamma}\right)=\frac{1}{n+2} \lambda_{A}^{0}, \\
F_{2 n+2,2 n+2}=0 .
\end{gathered}
$$


Next, using $F^{a b} F_{b c}=\delta_{c}^{a}$ (with $\left.a, b, c, \cdots \in\{1, \cdots, 2 n+2\}\right)$ we find

$$
\left\{\begin{array}{l}
F^{A B} F_{B C}+\frac{\lambda_{C}^{0}}{n+2} F^{A, 2 n+2}=\delta_{C}^{A} \\
F^{A B} \lambda_{B}^{0}=0 \\
F^{2 n+2, B} F_{B C}+\frac{\lambda_{C}^{0}}{n+2} F^{2 n+2,2 n+2}=0 \\
F^{2 n+2, B} \lambda_{B}^{0}=n+2 .
\end{array}\right.
$$

Let us set

$$
P_{A B}{ }^{i}{ }_{j} e_{i}=\left(R^{D} e_{j}\right)\left(T_{A}, T_{B}\right)
$$

so that $R_{A B j}^{i}=\lambda_{A}^{C} \lambda_{B}^{D} P_{C D}{ }^{i}{ }_{j}$. In the sequel, for the sake of simplicity, we do not distinguish notationally between $f \in C^{\infty}(M)$ and its vertical lift $f \circ \pi$. Then

$$
\begin{gathered}
\left\langle R^{\hat{D}}, R^{\hat{D}}\right\rangle=h^{j \bar{k}}\left\langle R^{\hat{D}} \hat{e}_{j}, R^{\hat{D}} \hat{e}_{k}\right\rangle= \\
=h^{j \bar{k}} h_{r \bar{s}} F_{\theta}^{*}\left(R_{A B j}^{r} d \hat{x}^{A} \wedge d \hat{x}^{B}, R_{C D k}^{s} d \hat{x}^{C} \wedge d \hat{x}^{D}\right)= \\
=\frac{1}{2} h^{j \bar{k}} h_{r \bar{s}} R_{A B j}^{r} R_{C D \bar{k}}^{\bar{s}}\left(F^{A C} F^{B D}-F^{A D} F^{B C}\right),
\end{gathered}
$$

where $R_{A B \bar{j}}^{\bar{i}}=\overline{R_{A B j}^{i}}$. We obtain

$$
\left\langle R^{\hat{D}}, R^{\hat{D}}\right\rangle=
$$

$$
=\frac{1}{2} h^{j \bar{k}} h_{r \bar{s}} \lambda_{A}^{E} \lambda_{B}^{F} \lambda_{C}^{\bar{G}} \lambda_{D}^{\bar{H}} P_{E F}{ }_{j}{ }_{j} P_{\bar{G}} \bar{H}^{\bar{s}}\left(F^{A C} F^{B D}-F^{A D} F^{B C}\right),
$$

where $\lambda_{A}^{\bar{B}}=\overline{\lambda_{A}^{B}}$ and $P_{\bar{A}} \bar{B}_{\bar{B}}^{\bar{j}}=\overline{P_{A B}{ }^{i}{ }_{j}}$. Note that $\lambda_{A}^{0}$ is real valued while $\lambda_{A}^{\bar{\alpha}}=\lambda_{A}^{\alpha+n}$. To calculate $\lambda_{A}^{E} \lambda_{B}^{F} \lambda_{C}^{\bar{G}} \lambda_{D}^{\bar{H}}\left(F^{A C} F^{B D}-F^{A D} F^{B C}\right)$ we need the identities

$$
\begin{gathered}
F^{A B} \lambda_{A}^{\alpha} \lambda_{B}^{\bar{\beta}}=g^{\alpha \bar{\beta}}, \\
F^{A B} \lambda_{A}^{\alpha} \lambda_{B}^{\beta}=0 .
\end{gathered}
$$

The proof of (77) $-(78)$ follows from (75). Indeed (75) may be written

$$
\begin{gathered}
F^{A B} g_{\alpha \bar{\beta}}\left(\lambda_{B}^{\alpha} \lambda_{C}^{\bar{\beta}}+\lambda_{C}^{\alpha} \lambda_{B}^{\bar{\beta}}\right)+F^{A B} \lambda_{C}^{0} \sigma_{B}+\frac{1}{n+2} F^{A, 2 n+2} \lambda_{C}^{0}=\delta_{C}^{A}, \\
F^{A B} \lambda_{B}^{0}=0, \\
F^{2 n+2, B} g_{\alpha \bar{\beta}}\left(\lambda_{B}^{\alpha} \lambda_{C}^{\bar{\beta}}+\lambda_{C}^{\alpha} \lambda_{B}^{\bar{\beta}}\right)+(n+2) \sigma_{C}+ \\
+F^{2 n+2, B} \lambda_{C}^{0} \sigma_{B}+\frac{1}{n+2} F^{2 n+2,2 n+2} \lambda_{C}^{0}=0,
\end{gathered}
$$




$$
F^{2 n+2, B} \lambda_{B}^{0}=n+2 .
$$

If $\mu:=\lambda^{-1}$ then (by the first of the previous four identities)

$$
\mu_{D}^{A}=\left(\frac{1}{n+2} F^{A, 2 n+2}+F^{A B} \sigma_{B}\right) \delta_{D}^{0}+F^{A B} g_{\alpha \bar{\beta}}\left(\lambda_{B}^{\alpha} \delta_{D}^{\beta+n}+\lambda_{B}^{\bar{\beta}} \delta_{D}^{\alpha}\right)
$$

yielding

$$
\left\{\begin{array}{l}
\mu_{0}^{A}=\frac{1}{n+2} F^{A, 2 n+2}+F^{A B} \sigma_{B} \\
\mu_{\alpha}^{A}=F^{A B} g_{\alpha \bar{\beta}} \lambda_{B}^{\bar{\beta}} \\
\mu_{\beta+n}^{A}=F^{A B} g_{\alpha \bar{\beta}} \lambda_{B}^{\alpha} .
\end{array}\right.
$$

The second and third of the identities (79) lead to (77) and (78), respectively. A calculation based on (77)-(78) shows that (766) may be written

$$
\begin{gathered}
\left\langle R^{\hat{D}}, R^{\hat{D}}\right\rangle= \\
=P^{\bar{\alpha} \bar{\beta} \bar{k} j} P_{\bar{\alpha} \bar{\beta} \bar{k} j}+P^{\alpha \beta \bar{k} j} P_{\alpha \beta \bar{k} j}+P^{\bar{\alpha} \beta \bar{k} j} P_{\bar{\alpha} \beta \bar{k} j}+P^{\alpha \bar{\beta} \bar{k} j} P_{\alpha \bar{\beta} \bar{k} j}
\end{gathered}
$$

where $P_{A B \bar{k} j}=h_{j \bar{s}} P_{A B \bar{k}}{ }^{\bar{s}}$ and $P^{A B \bar{k} j}=h^{r \bar{k}} P^{A B}{ }_{r}^{j}$. Also $P_{r}^{\alpha \beta}{ }_{r}^{j}=$ $g^{\alpha \bar{\lambda}} g^{\beta \bar{\mu}} P_{\bar{\lambda} \bar{\mu} r}{ }^{j}$, etc. As $R^{D} e_{j}=\left(P_{A B}{ }^{i}{ }_{j} \theta^{A} \wedge \theta^{B}\right) \otimes e_{i}$ it follows that

$$
\begin{aligned}
& \left\langle\pi_{H} R^{D}, \pi_{H} R^{D}\right\rangle=h^{j \bar{k}}\left\langle\left(\pi_{H} R^{D}\right) e_{j},\left(\pi_{H} R^{D}\right) e_{k}\right\rangle=
\end{aligned}
$$

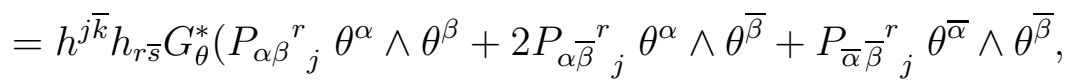

$$
\begin{aligned}
& \left.P_{\lambda \mu k}^{s} \theta^{\lambda} \wedge \theta^{\mu}+2 P_{\lambda \bar{\mu}}{ }^{s}{ }_{k} \theta^{\lambda} \wedge \theta^{\bar{\mu}}+P_{\bar{\lambda} \bar{\mu}}{ }_{k}^{s} \theta^{\bar{\lambda}} \wedge \theta^{\bar{\mu}}\right)= \\
& =\frac{1}{2} P_{\alpha \beta}{ }^{\bar{k} j} P_{\bar{\lambda} \bar{\mu} \bar{k} j}\left(g^{\alpha \bar{\lambda}} g^{\beta \bar{\mu}}-g^{\alpha \bar{\mu}} g^{\beta \bar{\lambda}}\right)+ \\
& +2 g^{\alpha \bar{\lambda}} g^{\bar{\beta} \mu} P_{\alpha \bar{\beta}}{ }^{\bar{k} j} P_{\bar{\lambda} \mu \bar{k} j}+ \\
& +\frac{1}{2} P_{\bar{\alpha} \bar{\beta}}{ }^{\bar{k} j} P_{\lambda \mu \bar{k} j}\left(g^{\bar{\alpha} \lambda} g^{\bar{\beta} \mu}-g^{\bar{\alpha} \mu} g^{\bar{\beta} \lambda}\right)= \\
& =P^{\bar{\lambda} \bar{\mu} \bar{k} j} P_{\bar{\lambda} \bar{\mu} \bar{k} j}+2 P^{\bar{\lambda} \mu \bar{k} j} P_{\bar{\lambda} \mu \bar{k} j}+P^{\lambda \mu \bar{k} j} P_{\lambda \mu \bar{k} j}
\end{aligned}
$$

hence (by (73) and (80))

$$
\left\langle R^{\hat{D}}, R^{\hat{D}}\right\rangle=\left(\left\|\pi_{H} R^{D}\right\| \circ \pi\right)^{2} .
$$

Finally we may integrate over $C(M)$ and use the identity

$$
\int_{C(M)}(f \circ \pi) d \operatorname{vol}\left(F_{\theta}\right)=2 \pi \int_{M} f \theta \wedge(d \theta)^{n}, f \in C^{\infty}(M) .
$$


The identity $^{1}$ (9) in Theorem 1 is proved. Assume now that $\hat{D}=\pi^{*} D$ is a Yang-Mills field on $C(M)$. Let $D^{t}=D+t \varphi, \varphi \in \Omega^{1}(\operatorname{Ad}(E))$, be a variation of $D$. Then

$$
\pi^{*} D^{t}=\hat{D}+t \pi^{*} \varphi
$$

A word on the conventions in (81). As seen earlier in this section, there is a natural map $\pi^{*}: \Omega^{1}(\operatorname{Ad}(E)) \rightarrow \Omega^{1}\left(\pi^{*} \operatorname{Ad}(E)\right)$. Yet $\operatorname{Ad}\left(\pi^{*} E\right) \approx$ $\pi^{*} \operatorname{Ad}(E)$ (a vector bundle isomorphism) hence $\pi^{*} \varphi$ is an $\operatorname{Ad}\left(\pi^{*} E\right)$ valued 1 -form on $C(M)$. Then (by (9))

$$
\begin{gathered}
0=\frac{d}{d t}\left\{\widehat{\mathcal{Y M}}\left(\hat{D}+t \pi^{*} \varphi\right)\right\}_{t=0}= \\
=\frac{d}{d t}\left\{\widehat{\mathcal{Y M}}\left(\pi^{*} D^{t}\right)\right\}_{t=0}=2 \pi \frac{d}{d t}\left\{\mathcal{P} \mathcal{Y \mathcal { M }}\left(D^{t}\right)\right\}_{t=0},
\end{gathered}
$$

i.e. $D$ is a pseudo Yang-Mills field on $M$. The converse requires the first variation formula for the functional $\mathcal{P} \mathcal{Y} \mathcal{M}$ (as well as the fact that the Yang-Mills equations on $C(M)$ project on $M$ via $\pi$ to give the Euler-Lagrange equations of the variational principle $\delta \mathcal{P} \mathcal{Y} \mathcal{M}=0$, cf. section 4). To establish (8) we need the following

Lemma 1. Let $M$ be a nondegenerate $C R$ manifold, $\theta$ a contact form on $M$, and $d \operatorname{vol}\left(g_{\theta}\right)$ the canonical volume form associated to the Webster metric $g_{\theta}$. Then $\theta \wedge(d \theta)^{n}= \pm c_{n} d \operatorname{vol}\left(g_{\theta}\right)$ where $c_{n}=(-1)^{s} 2^{n} n$ !, provided that the Levi form $L_{\theta}$ has $s$ negative eigenvalues.

This corrects the constant $c_{n}$ from 30, p. 546. If the Levi form $L_{\theta}$ has $r$ positive and $s$ negative eigenvalues $(r+s=n)$ then $g_{\theta}$ is a semi-Riemannian metric of signature $(2 r+1,2 s)$. Let $\mathcal{O}$ be a fixed orientation of $M$. To prove Lemma 1 let $G_{A B}$ be the components of the Webster metric with respect to a chart $\left(U, x^{A}\right) \in \mathcal{O}$, so that $d \operatorname{vol}\left(g_{\theta}\right)=\sqrt{\left|\operatorname{det}\left(G_{A B}\right)\right|} d x^{1} \wedge \cdots \wedge d x^{2 n+1}$. Let $\left\{T_{\alpha}\right\}$ be a local frame of $T_{1,0}(M)$ and $\mu \in G L(2 n+1, \mathbb{C})$ such that $T_{A}=\mu_{A}^{B} \partial / \partial x^{B}$. Then $d \operatorname{vol}\left(g_{\theta}\right)=\sqrt{\left|\operatorname{det}\left(G_{A B}\right)\right|} \operatorname{det}(\mu) \theta^{01 \cdots n \overline{1} \cdots \bar{n}}$, where $\theta^{01 \cdots n \overline{1} \cdots \bar{n}}$ is short for $\theta \wedge \theta^{1} \wedge \cdots \wedge \theta^{n} \wedge \theta^{\overline{1}} \wedge \cdots \wedge \theta^{\bar{n}}$, hence $\overline{\operatorname{det}(\mu)}=(-1)^{n^{2}} \operatorname{det}(\mu)\left(\operatorname{as} d \operatorname{vol}\left(g_{\theta}\right)\right.$ is a real form). It follows that $\sqrt{\left|\operatorname{det}\left(G_{A B}\right)\right|}=(-1)^{s}|\operatorname{det}(\mu)|^{-1} \operatorname{det}\left(g_{\alpha \bar{\beta}}\right)$. A calculation shows that

$$
\theta \wedge(d \theta)^{n}=2^{n} i^{n^{2}} n ! \operatorname{det}\left(g_{\alpha \bar{\beta}}\right) \theta^{01 \cdots n \overline{1} \cdots \bar{n}}
$$

(cf. also [19]) and then $\theta \wedge(d \theta)^{n}= \pm c_{n} d \operatorname{vol}\left(g_{\theta}\right)$. The sign is +1 if $\mathcal{O}$ and the orientation of $H(M)$ (induced by its complex structure $J$ ) agree. Lemma 1 is proved. Let us prove (8) . As

$$
R^{D} e_{j}=\left(\pi_{H} R^{D}\right) e_{j}-2 P_{0 A}{ }^{i}\left(\theta \wedge \theta^{A}\right) \otimes e_{i}
$$

\footnotetext{
${ }^{1}$ The symbol $\pi$ in the right hand side denotes the irrational number $\pi \in \mathbb{R}$.
} 
it follows that

$$
\left\|R^{D}\right\|^{2}=\left\|\pi_{H} R^{D}\right\|^{2}+4 g^{A \bar{B}} P_{0 A}{ }_{j}{ }_{j} P_{0 B}{ }_{i}{ }_{i} .
$$

Yet $i_{T} R^{D}=\theta^{B} \otimes\left[P_{0 B}{ }^{i}{ }_{j}\right] \in \Omega^{1}(\operatorname{Ad}(E))$ hence $\left\|R^{D}\right\|^{2}=\left\|\pi_{H} R^{D}\right\|^{2}+$ $4\left\|i_{T} R_{D}\right\|^{2}$. At this point we may integrate over $M$ with respect to $\theta \wedge(d \theta)^{n}$ and use Lemma 1. To proof of the last statement in i) of Theorem 2 is delegated to the next section.

\section{THE FIRST VARIATION FORMULA}

Let $E \rightarrow M$ be a vector bundle and $D$ a connection in $E$. We shall need the differential operator $d^{D}: \Omega^{k}(E) \rightarrow \Omega^{k+1}(E)$ given by

$$
\begin{gathered}
\left(d^{D} \varphi\right)\left(X_{1}, \cdots, X_{k+1}\right)=\sum_{i=1}^{k+1}(-1)^{i+1} D_{X_{i}}\left(\varphi\left(X_{1}, \cdots, \hat{X}_{i}, \cdots, X_{k+1}\right)\right)+ \\
\quad+\sum_{1 \leq i<j \leq k+1}(-1)^{i+j} \varphi\left(\left[X_{i}, X_{j}\right], X_{1}, \cdots, \hat{X}_{i}, \cdots, \hat{X}_{j}, \cdots, X_{k+1}\right)
\end{gathered}
$$

for any $\varphi \in \Omega^{k}(E)$ and any $X_{i} \in T(M), 1 \leq i \leq k$. Here a hat indicates, as usual, the suppression of a term. Let $D \in \mathcal{C}(E, h)$ and let us denote by the same symbol the connection induced by $D$ in $\operatorname{Ad}(E) \rightarrow M$. The operator $\delta^{D}$ in (18) is the formal adjoint of $d^{D}$ : $\Omega^{1}(\operatorname{Ad}(E)) \rightarrow \Omega^{2}(\operatorname{Ad}(E))$ with respect to the inner product

$$
(\varphi, \psi)=\int_{M}\langle\varphi, \psi\rangle \theta \wedge(d \theta)^{n}, \varphi, \psi \in \Omega^{k}(E) .
$$

Let $\varphi \in \Omega^{1}(\operatorname{Ad}(E))$. A standard calculation shows that $R^{D+t \varphi}=R^{D}+$ $t d^{D} \varphi+t^{2}[\varphi \wedge \varphi]\left(\right.$ where $[\varphi \wedge \psi]_{X, Y}=\left[\varphi_{X}, \psi_{Y}\right]-\left[\varphi_{Y}, \psi_{X}\right], X, Y \in T(M)$, $\left.\varphi, \psi \in \Omega^{1}(\operatorname{Ad}(E))\right)$ hence

$$
\left\|\pi_{H} R^{D+t \varphi}\right\|^{2}=\left\|\pi_{H} R^{D}\right\|^{2}+2 t\left\langle\pi_{H} R^{D}, \pi_{H} d^{D} \varphi\right\rangle+O\left(t^{2}\right)
$$

and

$$
\begin{gathered}
\frac{d}{d t}\{\mathcal{P} \mathcal{Y} \mathcal{M}(D+t \varphi)\}_{t=0}=\frac{1}{2} \int_{M} \frac{d}{d t}\left\{\left\|\pi_{H} R^{D+t \varphi}\right\|^{2}\right\}_{t=0} \theta \wedge(d \theta)^{n}= \\
=\int_{M}\left\langle\pi_{H} R^{D}, d^{D} \varphi\right\rangle \theta \wedge(d \theta)^{n}=\int_{M}\left\langle\delta^{D} \pi_{H} R^{D}, \varphi\right\rangle \theta \wedge(d \theta)^{n} .
\end{gathered}
$$

Then $\frac{d}{d t}\{\mathcal{P} \mathcal{Y} \mathcal{M}(D+t \varphi)\}_{t=0}=0$ yields

$$
\delta^{D} \pi_{H} R^{D}=0 .
$$

Let $D \in \mathcal{C}(E, h)$ such that $i_{T} R^{D}=0$. Then (by (18) and (83)) $D$ is a pseudo Yang-Mills field if and only if $D$ is a Yang-Mills field, and the last statement in part i) of Theorem 2 follows from Theorem 2.3 in 30, p. 551. 
Let us consider the operator $\delta_{b}^{D}: \Omega^{k+1}(E) \rightarrow \Omega^{k}(E)$ given by

$$
\left(\delta_{b}^{D} \varphi\right)\left(X_{1}, \cdots, X_{k}\right)=-\sum_{a=1}^{2 n}\left(D_{E_{a}} \varphi\right)\left(E_{a}, X_{1}, \cdots, X_{k}\right),
$$

for any $\varphi \in \Omega^{k+1}(E)$ and $X_{i} \in T(M), 1 \leq i \leq k$, where $\left\{E_{a}: 1 \leq a \leq\right.$ $2 n\}$ is a local $G_{\theta}$-orthonormal frame of $\bar{H}(M)$. Clearly, if $\varphi \in \bar{\Omega}_{H}^{k}(E)$ then $\delta^{D} \varphi=\delta_{b}^{D} \varphi$ and $i_{T} \delta_{b}^{D} \varphi=0$. Consequently, if $i_{T} R^{D}=0$ then the equations (83) may also be written

$$
\delta_{b}^{D} R^{D}=0 .
$$

Now we attack the problem whether the pullback $\hat{D}=\pi^{*} D$ of a pseudo Yang-Mills field $D$ on $M$ is a Yang-Mills field on $C(M)$. As argued in the previous section, this doesn't follow directly from (9). In turn, the Yang-Mills equations on $C(M)$ are related to (83) due to

$$
\begin{gathered}
\left(\delta^{\hat{D}} R^{\hat{D}}\right)\left(X^{\uparrow}\right) \hat{u}=\left(\left(\delta_{b}^{D} R^{D}\right)(X) u+R^{D}(T, J X) u\right)^{\wedge}, \\
\left(\delta^{\hat{D}} R^{\hat{D}}\right)\left(T^{\uparrow}\right) \hat{u}=\left(\left(\delta_{b}^{D} R^{D}\right)(T) u\right)^{\wedge}- \\
-\frac{i}{n+2}\left(R^{\alpha \bar{\beta}}-\frac{\rho}{2(n+1)} g^{\alpha \bar{\beta}}\right)\left(R^{D}\left(T_{\alpha}, T_{\bar{\beta}}\right) u\right)^{\wedge}, \\
\left(\delta^{\hat{D}} R^{\hat{D}}\right)(S) \hat{u}=2\left(\left(\Lambda_{\theta} R^{D}\right) u\right)^{\wedge},
\end{gathered}
$$

for any $X \in H(M)$ and $u \in \Omega^{0}(E)$. Here $X^{\uparrow}$ is the horizontal lift of $X$ with respect to the connection 1 -form $\sigma$ in $S^{1} \rightarrow C(M) \rightarrow M$. Let $D \in \mathcal{C}(E, h)$ be a pseudo Yang-Mills field with $i_{T} R^{D}=0$. Then (by (85)-(87) $) \delta^{\hat{D}} R^{\hat{D}}=0$ if and only if (10)-(11) hold. This completes the proof of Theorem 2 .

It remains that we prove (85)-(87). The formal adjoint $\delta^{\mathbb{D}}$ of $d^{\mathbb{D}}$ : $\Omega^{1}\left(\operatorname{Ad}\left(\pi^{*} E\right)\right) \rightarrow \Omega^{2}\left(\operatorname{Ad}\left(\pi^{*} E\right)\right)$ is given by

$$
\begin{gathered}
\left(\delta^{\mathbb{D}} \psi\right)(Y) v=-\sum_{j=1}^{2 n+2} \epsilon_{j}\left(\mathbb{D}_{X_{j}} \psi\right)\left(X_{j}, Y\right) v= \\
=-\sum_{j=1}^{2 n+2}\left(\mathbb{D}_{X_{j}} \psi\left(X_{j}, Y\right) v-\psi\left(\nabla_{X_{j}}^{C(M)} X_{j}, Y\right) v-\right. \\
\left.-\psi\left(X_{j}, \nabla_{X_{j}}^{C(M)} Y\right) v-\psi\left(X_{j}, Y\right) \mathbb{D}_{X_{j}} v\right),
\end{gathered}
$$

for any $\psi \in \Omega^{2}\left(\operatorname{Ad}\left(\pi^{*} E\right)\right), Y \in T(C(M))$ and $v \in \Omega^{0}\left(\pi^{*} E\right)$, where $\left\{X_{j}: 1 \leq j \leq 2 n+2\right\}$ is a local orthonormal (i.e. $F_{\theta}\left(X_{j}, X_{k}\right)=\epsilon_{j} \delta_{j k}$, $\left.\epsilon_{1}=\cdots=\epsilon_{2 n+1}=-\epsilon_{2 n+2}=1\right)$ frame of $T(C(M))$ and $\nabla^{C(M)}$ is 
the Levi-Civita connection of $\left(C(M), F_{\theta}\right)$. As $S^{1} \rightarrow C(M) \rightarrow M$ is a principal bundle, the projection $\pi$ is a submersion. However, if $S=((n+2) / 2) \partial / \partial \gamma$ then $F_{\theta}(S, S)=0$, i.e. $S$ is null, so that $\pi$ is not a semi-Riemannian submersion (in the sense of [22, p. 212, as the fibers of $\pi$ are degenerate submanifolds). Nevertheless, we may relate $\nabla^{C(M)}$ to the Tanaka-Webster connection $\nabla$ of $(M, \theta)$, very much in the spirit of [23]. Precisely, we may state

Lemma 2. For any $X, Y \in H(M)$

$$
\begin{gathered}
\nabla_{X^{\uparrow}}^{C(M)} Y^{\uparrow}=\left(\nabla_{X} Y\right)^{\uparrow}-(d \theta)(X, Y) T^{\uparrow}-\left(A(X, Y)+(d \sigma)\left(X^{\uparrow}, Y^{\uparrow}\right)\right) S, \\
\nabla_{X^{\uparrow}}^{C(M)} T^{\uparrow}=(\tau X+\phi X)^{\uparrow}, \\
\nabla_{T^{\uparrow}}^{C(M)} X^{\uparrow}=\left(\nabla_{T} X+\phi X\right)^{\uparrow}+2(d \sigma)\left(X^{\uparrow}, T^{\uparrow}\right) S, \\
\nabla_{X}^{C(M)} S=\nabla_{S}^{C(M)} X^{\uparrow}=(J X)^{\uparrow}, \\
\nabla_{T^{\uparrow}}^{C(M)} T^{\uparrow}=V^{\uparrow}, \quad \nabla_{S}^{C(M)} S=0, \\
\nabla_{S}^{C(M)} T^{\uparrow}=\nabla_{T^{\uparrow}}^{C(M)} S=0,
\end{gathered}
$$

where $\phi: H(M) \rightarrow H(M)$ is given by $G_{\theta}(\phi X, Y)=(d \sigma)\left(X^{\uparrow}, Y^{\uparrow}\right)$, and $V \in H(M)$ is given by $G_{\theta}(V, Y)=2(d \sigma)\left(T^{\uparrow}, Y^{\uparrow}\right)$.

Proof of Lemma Q Let us recall that

$$
2 F_{\theta}\left(\nabla_{\tilde{X}}^{C(M)} \tilde{Y}, \tilde{Z}\right)=\tilde{X}\left(F_{\theta}(\tilde{Y}, \tilde{Z})\right)+\tilde{Y}\left(F_{\theta}(\tilde{X}, \tilde{Z})\right)-\tilde{Z}\left(F_{\theta}(\tilde{X}, \tilde{Y})\right)+
$$

$$
+F_{\theta}([\tilde{X}, \tilde{Y}], \tilde{Z})+F_{\theta}([\tilde{Z}, \tilde{X}], \tilde{Y})+F_{\theta}(\tilde{X},[\tilde{Z}, \tilde{Y}])
$$

for any $\tilde{X}, \tilde{Y}, \tilde{Z} \in T(C(M))$. In particular for $\tilde{X}=X^{\uparrow}, \tilde{Y}=Y^{\uparrow}, \tilde{Z}=$ $Z^{\uparrow}$, for any $X, Y, Z \in H(M)$

$$
F_{\theta}\left(\nabla_{X^{\uparrow}}^{C(M)} Y^{\uparrow}, Z^{\uparrow}\right)=g_{\theta}\left(\nabla_{X}^{M} Y, Z\right),
$$

where $\nabla^{M}$ is the Levi-Civita connection of $\left(M, g_{\theta}\right)$. Here one used the fact that $[X, Y]^{\uparrow}$ is the horizontal component of $\left[X^{\uparrow}, Y^{\uparrow}\right]$, with respect to $\sigma$ (cf. e.g. [16], Vol. I, p. 65). The Levi-Civita connection $\nabla^{M}$ and the Tanaka-Webster connection $\nabla$ of $(M, \theta)$ are related by (cf. (1) in [1], p. 238)

$$
\nabla^{M}=\nabla-(d \theta+A) \otimes T+\tau \otimes \theta+2 \theta \odot J
$$

where $A(X, Y)=g_{\theta}(X, \tau Y)$. Recall that $A$ is symmetric and $\tau$ traceless (cf. 34). As $H(M)$ is $\nabla$-parallel $\pi_{H} \nabla_{X}^{M} Y=\nabla_{X} Y$, where $\pi_{H}$ : $T(M) \rightarrow H(M)$ is the projection associated with the direct sum decomposition $T(M)=H(M) \oplus \mathbb{R} T$. Therefore, by taking into account 
the decomposition $T(C(M))=\operatorname{Ker}(\sigma) \oplus \operatorname{Ker}(d \pi)=H(M)^{\uparrow} \oplus \mathbb{R} T^{\uparrow} \oplus \mathbb{R} S$

$$
\nabla_{X^{\uparrow}}^{C(M)} Y^{\uparrow}=\left(\nabla_{X} Y\right)^{\uparrow}+\lambda T^{\uparrow}+\mu S
$$

for some $\lambda, \mu \in C^{\infty}(C(M))$, depending on $X, Y$. We may determine $\lambda$, $\mu$ by taking the inner product with $S, T^{\uparrow}$, respectively. To this end let us first observe that

$$
F_{\theta}\left(\nabla_{X^{\uparrow}}^{C(M)} Y^{\uparrow}, S\right)=-(d \theta)(X, Y) .
$$

Here we used again (88) together with the fact that $\left[X^{\uparrow}, S\right]=0$ (cf. e.g. [16], Vol. I, p. 79). Similarly

$$
\begin{gathered}
2 F_{\theta}\left(\nabla_{X^{\uparrow}}^{C(M)} Y^{\uparrow}, T^{\uparrow}\right)=\sigma\left(\left[X^{\uparrow}, Y^{\uparrow}\right]\right)- \\
-T\left(g_{\theta}(X, Y)\right)+g_{\theta}([T, X], Y)+g_{\theta}(X,[T, Y])
\end{gathered}
$$

and

$$
\begin{gathered}
2 g_{\theta}\left(\nabla_{X}^{M} Y, T\right)=-T\left(g_{\theta}(X, Y)\right)+\theta([X, Y])+ \\
+g_{\theta}([T, X], Y)+g_{\theta}(X,[T, Y])
\end{gathered}
$$

hence

$$
2 F_{\theta}\left(\nabla_{X^{\uparrow}}^{C(M)} Y^{\uparrow}, T^{\uparrow}\right)=2 \theta\left(\nabla_{X}^{M} Y\right)-\theta([X, Y])+\sigma\left(\left[X^{\uparrow}, Y^{\uparrow}\right]\right)
$$

or (by (89))

$$
F_{\theta}\left(\nabla_{X^{\uparrow}}^{C(M)} Y^{\uparrow}, T^{\uparrow}\right)=-A(X, Y)-(d \sigma)\left(X^{\uparrow}, Y^{\uparrow}\right) .
$$

Summing up, (90) leads to the first identity in Lemma 2 . The proof of the remaining identities in Lemma 2 may be obtained in a similar manner. Let us go back to the proof of (85)-(87). Let $\left\{E_{a}: 1 \leq a \leq\right.$ $2 n\}$ be a local orthonormal frame of the Levi distribution $H(M)$. Then $\left\{E_{a}^{\uparrow}, T^{\uparrow} \pm S\right\}$ is a local orthonormal frame of $T(C(M))$ with respect to the Feferman metric $F_{\theta}$. We make use of $i_{S}(\hat{D} \hat{u})=0$ and $i_{S}\left(R^{\hat{D}} \hat{u}\right)=0$, for any $u \in \Omega^{0}(E)$. Then (by Lemma 2)

$$
\begin{gathered}
\left(\delta^{\hat{D}} R^{\hat{D}}\right)\left(X^{\uparrow}\right) \hat{u}=-\sum_{a=1}^{2 n}\left(\hat{D}_{E_{a}^{\uparrow}} R^{\hat{D}}\right)\left(E_{a}^{\uparrow}, X^{\uparrow}\right)- \\
-\left(\hat{D}_{T^{\uparrow}+S} R^{\hat{D}}\right)\left(T^{\uparrow}+S, X^{\uparrow}\right)+\left(\hat{D}_{T^{\uparrow}-S} R^{\hat{D}}\right)\left(T^{\uparrow}-S, X^{\uparrow}\right)= \\
=\left\{\left(\delta_{b}^{D} R^{D}\right)(X) u+2 R^{D}(T, J X) u\right\}^{\wedge}-\sum_{a=1}^{2 n}\left\{(d \theta)\left(E_{a}, X\right) R^{D}\left(E_{a}, T\right) u\right\}^{\wedge}
\end{gathered}
$$

and

$$
\sum_{a=1}^{2 n}(d \theta)\left(E_{a}, X\right) E_{a}=-J X
$$


hence (85) is proved. Similarly

$$
\left(\delta^{\hat{D}} R^{\hat{D}}\right)\left(T^{\uparrow}\right) \hat{u}=\left(\left(\delta_{b}^{D} R^{D}\right)(T) u\right)^{\wedge}+\sum_{a=1}^{2 n}\left\{R^{D}\left(E_{a}, \tau E_{a}+\phi E_{a}\right) u\right\}^{\wedge}
$$

Now, on one hand

$$
\begin{gathered}
\sum_{a=1}^{2 n} R^{D}\left(E_{a}, \tau E_{a}\right) u=g^{\alpha \bar{\beta}}\left\{R^{D}\left(T_{\alpha}, \tau T_{\bar{\beta}}\right) u+R^{D}\left(T_{\bar{\beta}}, \tau T_{\alpha}\right) u\right\}= \\
=A^{\alpha \gamma} R^{D}\left(T_{\alpha}, T_{\gamma}\right) u+A^{\bar{\beta} \bar{\gamma}} R^{D}\left(T_{\bar{\beta}}, T_{\bar{\gamma}}\right) u=0
\end{gathered}
$$

(as $A_{\alpha \beta}=A_{\beta \alpha}$ ) with the corresponding simplification of (91). On the other hand

$$
\begin{gathered}
\sum_{a=1}^{2 n} R^{D}\left(E_{a}, \phi E_{a}\right) u=\phi^{\alpha \gamma} R^{D}\left(T_{\alpha}, T_{\gamma}\right) u+\phi^{\alpha \bar{\gamma}} R^{D}\left(T_{\alpha}, T_{\bar{\gamma}}\right) u+ \\
+\phi^{\bar{\beta} \gamma} R^{D}\left(T_{\bar{\beta}}, T_{\gamma}\right) u+\phi^{\bar{\beta} \bar{\gamma}} R^{D}\left(T_{\bar{\beta}}, T_{\bar{\gamma}}\right) u
\end{gathered}
$$

where $\phi T_{\alpha}=\phi_{\alpha}{ }^{\beta} T_{\beta}+\phi_{\alpha}{ }^{\bar{\beta}} T_{\bar{\beta}}, \phi^{\alpha \beta}=g^{\alpha \bar{\gamma}} \phi_{\bar{\gamma}}{ }^{\beta}$, etc. Let us take the exterior derivative of (69) so that to obtain

$$
(n+2) d \sigma=\pi^{*}\left(i d \omega_{\alpha}^{\alpha}-\frac{i}{2} d g^{\alpha \bar{\beta}} \wedge d g_{\alpha \bar{\beta}}-\frac{1}{4(n+1)} d(\rho \theta)\right) .
$$

Using the identities $d g_{\alpha \bar{\beta}}=g_{\alpha \bar{\gamma}} \omega_{\bar{\beta}}^{\bar{\gamma}}+\omega_{\alpha}^{\gamma} g_{\gamma \bar{\beta}}$ (a consequence of $\nabla g_{\theta}=0$ ) and $d g^{\alpha \bar{\beta}}=-g^{\gamma \bar{\beta}} g^{\alpha \bar{\rho}} d g_{\bar{\rho} \gamma}$ (a consequence of $g^{\alpha \bar{\beta}} g_{\bar{\beta} \gamma}=\delta_{\gamma}^{\alpha}$ ) it follows that

$$
d g^{\alpha \bar{\beta}} \wedge d g_{\alpha \bar{\beta}}=\omega_{\alpha \bar{\beta}} \wedge \omega^{\alpha \bar{\beta}}+\omega_{\bar{\alpha} \beta} \wedge \omega^{\bar{\alpha} \beta}=0 .
$$

Also (cf. e.g. 34])

$$
d \omega_{\alpha}^{\alpha}=R_{\lambda \bar{\mu}} \theta^{\alpha} \wedge \theta^{\bar{\mu}}+\left(W_{\alpha \lambda}^{\alpha} \theta^{\lambda}-W_{\alpha \bar{\mu}}^{\alpha} \theta^{\bar{\lambda}}\right) \wedge \theta
$$

where $R_{\lambda \bar{\mu}}$ is the pseudohermitian Ricci curvature and $W_{\alpha \lambda}^{\alpha}$ (respectively $\left.W_{\alpha \bar{\mu}}^{\alpha}\right)$ are certain contractions of the covariant derivatives of $A_{\bar{\beta}}^{\alpha}$. It follows that

$$
(n+2) G_{\theta}(\phi X, Y)=i\left(R_{\alpha \bar{\beta}} \theta^{\alpha} \wedge \theta^{\bar{\beta}}\right)(X, Y)-\frac{\rho}{4(n+1)}(d \theta)(X, Y),
$$

for any $X, Y \in H(M)$. Therefore

$$
\phi^{\bar{\alpha} \beta}=\frac{i}{2(n+2)}\left(R^{\bar{\alpha} \beta}-\frac{\rho}{2(n+1)} g^{\bar{\alpha} \beta}\right), \quad \phi^{\alpha \beta}=0 .
$$

We may conclude that

$$
\sum_{A=1}^{2 n} R^{D}\left(E_{a}, \phi E_{a}\right) u=-\frac{i}{n+2}\left(R^{\alpha \bar{\beta}}-\frac{\rho}{2(n+1)} g^{\alpha \bar{\beta}}\right) R^{D}\left(T_{\alpha}, T_{\bar{\beta}}\right) u
$$


and (91) leads to (86). Finally (again by Lemma 21)

$$
\begin{gathered}
\left(\delta^{\hat{D}} R^{\hat{D}}\right)(S) \hat{u}=\sum_{a=1}^{2 n}\left(R^{D}\left(E_{a}, J E_{a}\right) u\right)^{\wedge}= \\
=-2 i\left\{g^{\alpha \bar{\beta}} R^{D}\left(T_{\alpha}, T_{\bar{\beta}}\right) u\right\}^{\wedge}=2\left\{\left(\Lambda_{\theta} R^{D}\right) u\right\}^{\wedge}
\end{gathered}
$$

and (87) is proved.

\section{THE SECOND VARIATION FORMULA}

Let $\left\{D^{t}:|t|<\epsilon\right\}$ be a smooth family of connections in $E$, where $D=D^{0}$ is a pseudo Yang-Mills field. We write $D^{t}=D+A^{t}$, where $A^{t} \in \Omega^{1}(\operatorname{Ad}(E))$ for each $|t|<\epsilon$. The curvature $R^{t}$ of $D^{t}$ is then given by

$$
R^{t}=R^{D}+d^{D} A^{t}+\frac{1}{2}\left[A^{t} \wedge A^{t}\right]
$$

(cf. e.g. (6.2) in [4, p. 212). Next, let us set $\varphi=\left\{d A^{t} / d t\right\}_{t=0}$ and $\psi=\left\{d^{2} A^{t} / d t^{2}\right\}_{t=0}$ and observe that

$$
\begin{gathered}
\left\|\pi_{H} R^{t}\right\|^{2}=\left\|\pi_{H} R^{D}\right\|^{2}+2 t\left\langle\pi_{H} R^{D}, d^{D} \varphi\right\rangle+ \\
+t^{2}\left\{2\left\langle\pi_{H} R^{D}, d^{D} \psi\right\rangle+\left\langle\pi_{H} R^{D},[\varphi \wedge \varphi]\right\rangle+\left\|\pi_{H} d^{D} \varphi\right\|^{2}\right\}+O\left(t^{3}\right) .
\end{gathered}
$$

Integrating by parts and using $\delta^{D} \pi_{H} R^{D}=0$ we obtain

$$
\begin{gathered}
\frac{d^{2}}{d t^{2}}\left\{\mathcal{P} \mathcal{Y} \mathcal{M}\left(D^{t}\right)\right\}_{t=0}= \\
=\int_{M}\left\{\left\|\pi_{H} d^{D} \varphi\right\|^{2}+\left\langle\pi_{H} R^{D},[\varphi \wedge \varphi]\right\rangle\right\} \theta \wedge(d \theta)^{n} .
\end{gathered}
$$

We shall need the (zero order) operator $\mathcal{R}^{D}: \Omega^{1}(\operatorname{Ad}(E)) \rightarrow \Omega^{1}(\operatorname{Ad}(E))$ given by

$$
\mathcal{R}^{D}(\varphi)_{X}=\sum_{A=1}^{2 n+1}\left[R_{E_{A}, X}^{D}, \varphi_{E_{A}}\right],
$$

for any $X \in T(M), \varphi \in \Omega^{1}(\operatorname{Ad}(E))$, where $\left\{E_{A}\right\}$ is a local orthonormal frame of $\left(T(M), g_{\theta}\right)$. Then (cf. (6.7) in [4], p. 213)

$$
\left\langle[\varphi \wedge \varphi], R^{D}\right\rangle=\left\langle\varphi, \mathcal{R}^{D}(\varphi)\right\rangle \text {. }
$$

Let us set

$$
\mathcal{R}_{b}^{D}(\varphi)_{X}=\sum_{a=1}^{2 n}\left[R_{E_{a}, X}^{D}, \varphi_{E_{a}}\right], \quad \mathcal{R}_{0}^{D}(\varphi)_{X}=\left[R_{T, X}^{D}, \varphi_{T}\right],
$$

where $\left\{E_{a}\right\}$ is a local orthonormal frame of $\left(H(M), G_{\theta}\right)$, so that $\mathcal{R}^{D}=$ $\mathcal{R}_{b}^{D}+\mathcal{R}_{0}^{D}$. Taking into account the identities

$$
[\varphi \wedge \varphi] e_{j}=4\left(\varphi_{k}^{i} \wedge \varphi_{j}^{k}\right) \otimes e_{i}
$$


38

$$
\begin{aligned}
& \left(\theta \wedge i_{T} R^{D}\right) e_{j}=\left(P_{0 A^{i}}{ }_{j} \theta \wedge \theta^{A}\right) \otimes e_{i}, \\
& \mathcal{R}_{0}^{D}(\varphi) e_{j}=\left\{\varphi_{j}^{k}(T) P_{0 A}{ }^{i}{ }_{k}-P_{0 A}{ }_{j}{ }_{j} \varphi_{k}^{i}(T)\right\} \theta^{A} \otimes e_{i},
\end{aligned}
$$

where $\varphi e_{j}=\varphi_{j}^{i} \otimes e_{i}, \varphi_{j}^{i} \in \Omega^{1}(U)$, we may conduct the calculations

$$
\begin{gathered}
\left\langle[\varphi \wedge \varphi], \theta \wedge i_{T} R^{D}\right\rangle=h^{j \bar{r}}\left\langle[\varphi \wedge \varphi] e_{j},\left(\theta \wedge i_{T} R^{D}\right) e_{r}\right\rangle= \\
=4 h^{j \bar{r}} h_{i \bar{s}} g_{\theta}^{*}\left(\varphi_{k}^{i} \wedge \varphi_{j}^{k}, P_{0 A^{s}}{ }_{r} \theta \wedge \theta^{A}\right)= \\
=2 h^{j \bar{r}} h_{i \bar{s}} g^{A \bar{B}}\left\{\varphi_{k}^{i}(T) \varphi_{j}^{k}\left(T_{A}\right)-\varphi_{k}^{i}\left(T_{A}\right) \varphi_{j}^{k}(T)\right\} P_{0 \bar{B}}{ }^{\bar{s}} \bar{r}
\end{gathered}
$$

and

$$
\begin{gathered}
\left\langle\varphi, \mathcal{R}_{0}^{D}(\varphi)\right\rangle=h^{j \bar{r}} h_{i \bar{s}} \varphi_{j}^{i}\left(T_{A}\right)\left\{\varphi_{\bar{r}}^{\bar{k}}(T) P_{0 \bar{B}^{\bar{s}} \bar{k}}-P_{0 \bar{B}}{ }^{\bar{k}} \varphi_{\bar{k}}^{\bar{s}}(T)\right\} g^{A \bar{B}}= \\
=\varphi_{j}^{i}\left(T_{A}\right) g^{A \bar{B}}\left\{h^{j \bar{k}} h_{i \bar{s}} \varphi_{\bar{k}}^{\bar{r}}(T)-h^{j \bar{r}} h_{i \bar{k}} \varphi_{\frac{\bar{s}}{s}}(T)\right\} P_{0 \bar{B}^{\bar{s}}{ }_{\bar{r}}} .
\end{gathered}
$$

Assume now that $\left\{e_{j}\right\}$ is orthonormal $\left(h_{i \bar{j}}=\delta_{i j}\right)$, so that $\varphi \frac{\bar{i}}{j}=-\varphi_{i}^{j}$ (as $\varphi$ is $\operatorname{Ad}(E)$-valued). Then

$$
\begin{gathered}
\left\langle[\varphi \wedge \varphi], \theta \wedge i_{T} R^{D}\right\rangle=2 \sum_{i, j}\left\{\varphi_{k}^{i}(T) \varphi_{j}^{k}\left(T_{A}\right)-\varphi_{k}^{i}\left(T_{A}\right) \varphi_{j}^{k}(T)\right\} g^{A \bar{B}} P_{0 \bar{B}} \overline{\bar{j}} \bar{j} \\
\left\langle\varphi, \mathcal{R}_{0}^{D}(\varphi)\right\rangle=\sum_{r, s}\left\{\varphi_{i}^{s}(T) \varphi_{r}^{i}\left(T_{A}\right)-\varphi_{j}^{s}\left(T_{A}\right) \varphi_{r}^{j}(T)\right\} g^{A \bar{B}} P_{0 \bar{B}} \bar{s}
\end{gathered}
$$

hence

$$
\left\langle[\varphi \wedge \varphi], \theta \wedge i_{T} R^{D}\right\rangle=2\left\langle\varphi, \mathcal{R}_{0}^{D}(\varphi)\right\rangle .
$$

Finally, let us take into account (93) and the identity

$$
R^{D}=\pi_{H} R^{D}+2 \theta \wedge i_{T} R^{D} .
$$

We obtain

$$
\left\langle[\varphi \wedge \varphi], \pi_{H} R^{D}\right\rangle=\left\langle\varphi, \mathcal{R}^{D}(\varphi)-4 \mathcal{R}_{0}^{D}(\varphi)\right\rangle,
$$

so that (92) becomes

$$
\begin{gathered}
\frac{d^{2}}{d t^{2}}\left\{\mathcal{P} \mathcal{Y} \mathcal{M}\left(D^{t}\right)\right\}_{t=0}= \\
=\int_{M}\left\langle\delta^{D} \pi_{H} d^{D} \varphi+\mathcal{R}^{D}(\varphi)-4 \mathcal{R}_{0}^{D}(\varphi), \varphi\right\rangle \theta \wedge(d \theta)^{n} .
\end{gathered}
$$

We now restrict our variations to those whose first order part $\varphi$ satisfies $i_{T} \varphi=0$ and $\delta^{D} \varphi=0$. Also, let us introduce the first order differential operator $d_{b}^{D}: \Omega^{1}(\operatorname{Ad}(E)) \rightarrow \Omega_{H}^{2}(\operatorname{Ad}(E))$ given by $d_{b}^{D} \equiv \pi_{H} \circ d^{D}$. Then $\delta^{D} \pi_{H} d^{D} \varphi=\delta_{b}^{D} d_{b}^{D} \varphi=\Delta_{b}^{D} \varphi$ and $\mathcal{R}_{0}^{D}(\varphi)=0$, so that (94) yields (12) in Theorem 3 , Here $\Delta_{b}^{D} \equiv d_{b}^{D} \delta_{b}^{D}+\delta_{b}^{D} d_{b}^{D}$ is the generalized sublaplacian. The Riemannian counterpart $\mathcal{S}^{D}=\Delta^{D}+\mathcal{R}^{D}$ (cf. 4, p. 213, where $\Delta^{D}$ is the generalized Hodge-de Rham laplacian) of $\mathcal{S}_{b}^{D}=\Delta_{b}^{D}+\mathcal{R}_{b}^{D}$ in Theorem 3 is an elliptic operator, hence its restriction to $\operatorname{Ker}\left(\delta^{D}\right) \subset$ $\Omega^{1}(\operatorname{Ad}(E))$ has a discrete spectrum tending to $+\infty$ and the eigenspace 
corresponding to each eigenvalue of $\mathcal{S}^{D}$ is finite dimensional. This allows one to employ concepts from Morse theory (cf. Definition 6.10 in 4, p. 213) in order to discuss stability and weak stability of YangMills fields (cf. 4, p. 214). The CR analog of this phenomenon is that $\Delta_{b}^{D}: \Omega^{0,1}(\operatorname{Ad}(E)) \rightarrow \Omega^{0,1}(\operatorname{Ad}(E))$ is subelliptic of order $1 / 2$, where $\Omega^{0, q}(\operatorname{Ad}(E))=\Gamma^{\infty}\left(\Lambda^{0, q}(M) \otimes \operatorname{Ad}(E)\right)$. A complex valued $q$-form $\eta$ on $M$ is of type $(0, q)$, or a $(0, q)$-form, if $\left.T_{1,0}(M)\right\rfloor \eta=0$ and $i_{T} \eta=0$. We denote by $\Lambda^{0, q}(M) \rightarrow M$ the relevant bundle and set $\Omega^{0, q}(M)=$ $\Gamma^{\infty}\left(\Lambda^{0, q}(M)\right)$. Let $M$ be a strictly pseudoconvex $\mathrm{CR}$ manifold (not necessarily compact). It is the proper place to recall that a formally self adjoint second order differential operator $L: C^{\infty}(M) \rightarrow C^{\infty}(M)$ is subelliptic (of order $0<\epsilon \leq 1$ ) at a point $x \in M$ if there is a neighborhood $U$ of $x$ such that

$$
\|u\|_{\epsilon}^{2} \leq C\left((L u, u)+\|u\|^{2}\right)
$$

for any $u \in C_{0}^{\infty}(U)$, where $\|u\|_{\epsilon}$ is the Sobolev norm $u$ of order $\epsilon$, $\|u\|=(u, u)^{1 / 2}$, and

$$
(u, v)=\int_{M} u v d \operatorname{vol}\left(g_{\theta}\right)
$$

is the ordinary $L^{2}$ inner product. $L$ is subelliptic (of order $\epsilon$ ) if it is subelliptic at any $x \in M$. A typical example is the sublaplacian

$$
\Delta_{b} u=-\operatorname{div}\left(\nabla^{H} u\right), \quad u \in C^{\infty}(M),
$$

where $\nabla^{H} u \equiv \pi_{H} \nabla u, \nabla u$ is the gradient of $u$ with respect to the Webster metric $g_{\theta}$, and the divergence is defined with respect to the volume form $\omega \equiv \theta \wedge(d \theta)^{n}$, i.e.

$$
\mathcal{L}_{X} \omega=\operatorname{div}(X) \omega
$$

for any $X \in \mathcal{X}(M)$, where $\mathcal{L}_{X}$ is the Lie derivative. It is easily seen that $\Delta_{b} u=-\sum_{a=1}^{2 n} E_{a}^{*} E_{a} u$, for any local orthonormal frame $\left\{E_{a}\right\}$ of $H(M)$ hence, by a well known lemma of E.V. Radkevic, [27, it follows that $\|u\|_{1 / 2}^{2} \leq C\left(\left(\Delta_{b} u, u\right)+\|u\|^{2}\right)$, for any $u \in C_{0}^{\infty}(U)$, i.e. $\Delta_{b}$ is subelliptic of order $1 / 2$. Here $E_{a}^{*}$ is the formal adjoint of $E_{a}$ with respect to the inner product (95). In the next section we relate $\Delta_{b}^{D}$ to the Kohn-Rossi operator $\square_{b}$ and explain the subellipticity of $\square_{b}$ on $(0,1)$-forms.

\section{Subellipticity of $\Delta_{b}^{D}$}

Let $\left\{T_{\alpha}\right\}$ be a local frame of $T_{1,0}(M)$. We start by computing

$$
\left(\delta_{b}^{D} d_{b}^{D} \varphi\right) T_{\lambda}=-\sum_{a=1}^{2 n}\left(D_{E_{a}} d_{b}^{D} \varphi\right)\left(E_{a}, T_{\lambda}\right)
$$


for any $\varphi \in \Omega_{H}^{1}(\operatorname{Ad}(E))$. Let us take into account the identities

$$
\begin{gathered}
g^{\alpha \bar{\beta}}\left(D_{T_{\alpha}} d_{b}^{D} \varphi\right)\left(T_{\bar{\beta}}, T_{\lambda}\right)= \\
=g^{\alpha \bar{\beta}}\left\{D_{T_{\alpha}}\left(\left(d_{b}^{D} \varphi\right)\left(T_{\bar{\beta}}, T_{\lambda}\right)\right)-\Gamma_{\alpha \bar{\beta}}^{\bar{\gamma}}\left(d_{b}^{D} \varphi\right)\left(T_{\bar{\gamma}}, T_{\lambda}\right)-\Gamma_{\alpha \lambda}^{\gamma}\left(d_{b}^{D} \varphi\right)\left(T_{\bar{\beta}}, T_{\gamma}\right)\right\}, \\
\left(d_{b}^{D} \varphi\right)\left(T_{\alpha}, T_{\bar{\beta}}\right) e_{j}=\left\{\left(\nabla_{T_{\alpha}} \varphi_{j}^{i}\right) T_{\bar{\beta}}-\left(\nabla_{T_{\bar{\beta}}} \varphi_{j}^{i}\right) T_{\alpha}+\right. \\
\left.+2\left(\omega_{k}^{i} \wedge \varphi_{j}^{k}+\varphi_{k}^{i} \wedge \omega_{j}^{k}\right)\left(T_{\alpha}, T_{\bar{\beta}}\right)\right\} e_{i},
\end{gathered}
$$

where $\omega_{j}^{i}$ are the connection 1 -forms of $D$ with respect to $\left\{e_{i}\right\}$. Also, let us set

$$
\nabla_{\bar{\mu}} \nabla_{\lambda} \psi_{\bar{\alpha}} \equiv\left(\nabla_{T_{\bar{\mu}}} \nabla \psi\right)\left(T_{\lambda}, T_{\bar{\alpha}}\right)=T_{\bar{\mu}}\left(\nabla_{\lambda} \psi_{\bar{\alpha}}\right)-\Gamma_{\bar{\mu} \lambda}^{\rho} \nabla_{\rho} \psi_{\bar{\alpha}}-\Gamma_{\bar{\mu}}^{\bar{\rho}} \psi_{\bar{\rho}},
$$

for any $\psi=\psi_{\bar{\alpha}} \theta^{\bar{\alpha}} \in \Omega^{0,1}(M)$, where $\nabla_{\alpha} \psi_{\bar{\beta}} \equiv\left(\nabla_{T_{\alpha}} \psi\right) T_{\bar{\beta}}$. We obtain

$$
\begin{aligned}
g^{\alpha \bar{\beta}}\left(D_{T_{\alpha}}\right. & \left.d_{b}^{D} \varphi\right)\left(T_{\bar{\beta}}, T_{\lambda}\right) e_{j}=-g^{\alpha \bar{\beta}}\left\{\nabla_{\alpha} \nabla_{\lambda} \varphi_{j \bar{\beta}}^{i}-\nabla_{\alpha} \nabla_{\bar{\beta}} \varphi_{j \lambda}^{i}+\right. \\
+ & \omega_{j}^{k}\left(T_{\bar{\beta}}\right) A_{k}^{i}\left(T_{\alpha}, T_{\lambda}\right)-\omega_{j}^{k}\left(T_{\lambda}\right) A_{k}^{i}\left(T_{\alpha}, T_{\bar{\beta}}\right)+ \\
+ & \omega_{k}^{i}\left(T_{\lambda}\right) B_{j}^{k}\left(T_{\alpha}, T_{\bar{\beta}}\right)-\omega_{k}^{i}\left(T_{\bar{\beta}}\right) B_{j}^{k}\left(T_{\alpha}, T_{\lambda}\right)+ \\
& +\omega_{k}^{i}\left(T_{\alpha}\right)\left[\left(\nabla_{T_{\lambda}} \varphi_{j}^{k}\right) T_{\bar{\beta}}-\left(\nabla_{T_{\bar{\beta}}} \varphi_{j}^{k}\right) T_{\lambda}\right]+ \\
& +\omega_{j}^{k}\left(T_{\alpha}\right)\left[\left(\nabla_{T_{\bar{\beta}}} \varphi_{k}^{i}\right) T_{\lambda}-\left(\nabla_{T_{\lambda}} \varphi_{k}^{i}\right) T_{\bar{\beta}}\right]+ \\
& +\varphi_{j \bar{\beta}}^{k} C_{k}^{i}\left(T_{\alpha}, T_{\lambda}\right)-\varphi_{j \lambda}^{k} C_{k}^{i}\left(T_{\alpha}, T_{\bar{\beta}}\right)+ \\
& \left.+\varphi_{k \lambda}^{i} D_{j}^{k}\left(T_{\alpha}, T_{\bar{\beta}}\right)-\varphi_{k \bar{\beta}}^{i} D_{j}^{k}\left(T_{\alpha}, T_{\bar{\beta}}\right)\right\} \otimes e_{i}
\end{aligned}
$$

where

$$
\begin{array}{ll}
A_{j}^{i} \equiv \nabla \varphi_{j}^{i}+\omega_{k}^{i} \otimes \varphi_{j}^{k}, & B_{j}^{i} \equiv \nabla \varphi_{j}^{i}-\omega_{j}^{k} \otimes \varphi_{k}^{i}, \\
C_{j}^{i} \equiv \nabla \omega_{j}^{i}+\omega_{k}^{i} \otimes \omega_{j}^{k}, & D_{j}^{i} \equiv \nabla \omega_{j}^{i}-\omega_{j}^{k} \otimes \omega_{k}^{i},
\end{array}
$$

and $\varphi_{j A}^{i}=\varphi_{j}^{i}\left(T_{A}\right)$, so that $\nabla_{\alpha} \nabla_{\bar{\beta}} \varphi_{j \lambda}^{i}$ (respectively $\nabla_{\alpha} \nabla_{\beta} \varphi_{j \bar{\lambda}}^{i}$ ) is the second order covariant derivative of the $(1,0)$-form $\pi_{1,0} \varphi_{j}^{i}$ (respectively of the $(0,1)$-form $\left.\pi_{0,1} \varphi_{j}^{i}\right)\left(\pi_{1,0}: \Omega^{1}(M) \rightarrow \Omega^{1,0}(M)\right.$ and $\pi_{0,1}: \Omega^{1}(M) \rightarrow$ $\Omega^{0,1}(M)$ are the natural projections). The previous identity is rather involved, yet one is interested in the second order terms alone. Together with the similar expression for $g^{\alpha \bar{\beta}}\left(D_{T_{\bar{\beta}}} d_{b}^{D} \varphi\right)\left(T_{\alpha}, T_{\lambda}\right) e_{j}$ this leads to

$$
\begin{aligned}
& \left(\delta_{b}^{D} d_{b}^{D} \varphi\right)\left(T_{\lambda}\right) e_{j}=g^{\alpha \bar{\beta}}\left(\nabla_{\alpha} \nabla_{\lambda} \varphi_{j \bar{\beta}}^{i}+\nabla_{\bar{\beta}} \nabla_{\lambda} \varphi_{j \alpha}^{i}-\right. \\
- & \left.\nabla_{\alpha} \nabla_{\bar{\beta}} \varphi_{j \lambda}^{i}-\nabla_{\bar{\beta}} \nabla_{\alpha} \varphi_{j \lambda}^{i}\right) e_{i}+\text { lower order terms. }
\end{aligned}
$$

By lower order terms (l.o.t.) we mean a linear combination of $\nabla_{A} \varphi_{j B}^{i}$ and $\varphi_{j B}^{i}$ (with $C^{\infty}(U)$-coefficients). Next, we need to compute

$$
\left(d_{b}^{D} \delta_{b}^{D} \varphi\right) T_{\lambda}=D_{T_{\lambda}}\left(\delta_{b}^{D} \varphi\right)=-\sum_{a=1}^{2 n} D_{T_{\lambda}}\left(\left(D_{E_{a}} \varphi\right) E_{a}\right) .
$$


We have

$$
\begin{gathered}
\quad\left[D_{T_{\lambda}}\left(g^{\alpha \bar{\beta}}\left(D_{T_{\alpha}} \varphi\right) T_{\bar{\beta}}\right)\right] e_{j}=g^{\alpha \bar{\beta}}\left\{\nabla_{\lambda} \nabla_{\alpha} \varphi_{j \bar{\beta}}^{i}+\right. \\
-\omega_{j}^{k}\left(T_{\lambda}\right) A_{k}^{i}\left(T_{\alpha}, T_{\bar{\beta}}\right)+\omega_{k}^{i}\left(T_{\lambda}\right) B_{j}^{k}\left(T_{\alpha}, T_{\bar{\beta}}\right)- \\
-\omega_{k}^{j}\left(T_{\alpha}\right)\left(\nabla_{T_{\lambda}} \varphi_{k}^{i}\right) T_{\bar{\beta}}+\omega_{k}^{i}\left(T_{\alpha}\right)\left(\nabla_{T_{\lambda}} \varphi_{j}^{k}\right) T_{\bar{\beta}}+ \\
\left.\quad+\varphi_{j \bar{\beta}}^{k} C_{k}^{i}\left(T_{\lambda}, T_{\alpha}\right)-\varphi_{k \bar{\beta}}^{i} D_{j}^{k}\left(T_{\lambda}, T_{\alpha}\right)\right\} e_{i} .
\end{gathered}
$$

Together with the similar expression for $D_{T_{\lambda}}\left(g^{\alpha \bar{\beta}}\left(D_{T_{\bar{\beta}}} \varphi\right) T_{\alpha}\right)$ this yields

$$
\left(d_{b}^{D} \delta_{b}^{D} \varphi\right)\left(T_{\lambda}\right) e_{j}=-g^{\alpha \bar{\beta}}\left\{\nabla_{\lambda} \nabla_{\alpha} \varphi_{j \bar{\beta}}^{i}+\nabla_{\lambda} \nabla_{\bar{\beta}} \varphi_{j \alpha}^{i}\right\} e_{i}+\text { l.o.t. }
$$

We shall need the commutation formulae

$$
\begin{gathered}
\nabla_{\alpha} \nabla_{\beta} \eta_{\bar{\gamma}}-\nabla_{\beta} \nabla_{\alpha} \eta_{\bar{\gamma}}=-\eta_{\bar{\rho}} R_{\bar{\gamma}}^{{ }^{\bar{\rho}}}{ }_{\alpha \beta} \\
\nabla_{\bar{\beta}} \nabla_{\alpha} \eta_{\gamma}-\nabla_{\alpha} \nabla_{\bar{\beta}} \eta_{\gamma}=2 i g_{\alpha \bar{\beta}} \nabla_{0} \eta_{\gamma}-\eta_{\rho} R_{\gamma}{ }^{\rho} \bar{\beta} \alpha
\end{gathered}
$$

where the convention for the curvature components (of the TanakaWebster connection) is $R\left(T_{A}, T_{B}\right) T_{C}=R_{C}{ }^{D}{ }_{A B} T_{D}$. Then (by (96)-(97))

$$
\begin{gathered}
\left(\Delta_{b}^{D} \varphi\right)\left(T_{\lambda}\right) e_{j}=g^{\alpha \bar{\beta}}\left(\nabla_{\alpha} \nabla_{\lambda} \varphi_{j \bar{\beta}}^{i}-\nabla_{\lambda} \nabla_{\alpha} \varphi_{j \bar{\beta}}^{i}+\right. \\
\left.+\nabla_{\bar{\beta}} \nabla_{\lambda} \varphi_{j \alpha}^{i}-\nabla_{\lambda} \nabla_{\bar{\beta}} \varphi_{j \alpha}^{i}-\nabla_{\alpha} \nabla_{\bar{\beta}} \varphi_{j \lambda}^{i}-\nabla_{\bar{\beta}} \nabla_{\alpha} \varphi_{j \lambda}^{i}\right) e_{i}+\text { l.o.t. }= \\
=\left\{-\varphi_{j}^{i}\left(T_{\bar{\rho}}\right) g^{\alpha \bar{\beta}} R_{\bar{\beta}{ }^{\bar{\rho}}}{ }_{\alpha \lambda}+2 \sqrt{-1} \nabla_{0} \varphi_{j \lambda}^{i}-\varphi_{j}^{i}\left(T_{\rho}\right) g^{\alpha \bar{\beta}} R_{\alpha}{ }^{\rho}{ }_{\bar{\beta} \lambda}-\right. \\
\left.-2 g^{\alpha \bar{\beta}} \nabla_{\bar{\beta}} \nabla_{\alpha} \varphi_{j \lambda}^{i}+2 \sqrt{-1} n \nabla_{0} \varphi_{j \lambda}^{i}-\varphi_{j}^{i}\left(T_{\rho}\right) g^{\alpha \bar{\beta}} R_{\lambda}{ }^{\rho}{ }_{\bar{\beta} \alpha}\right\} e_{i}+\text { l.o.t. }
\end{gathered}
$$

At this point we need the Kohn-Rossi operator $\square_{b}$ on $\Omega^{0,1}(M)$. We start by extending $\bar{\partial}_{b}$ (originally defined on functions, cf. section 3 ) to $(0,1)$-forms. Precisely, if $\eta \in \Omega^{0,1}(M)$ then $\bar{\partial}_{b} \eta$ is the unique $(0,2)$ form on $M$ coinciding with $d \eta$ on $T_{0,1}(M) \otimes T_{0,1}(M)$. Next, let us set $\square_{b} \equiv \bar{\partial}_{b}^{*} \bar{\partial}_{b}+\bar{\partial}_{b} \bar{\partial}_{b}^{*}$, where $\bar{\partial}_{b}^{*}$ is the formal adjoint of $\bar{\partial}_{b}$ with respect to the $L^{2}$ inner product $(\alpha, \beta)=\int_{M} g_{\theta}^{*}(\alpha, \bar{\beta}) \omega$. A straightforward calculation leads to

$$
\square_{b} \eta=\left(-g^{\alpha \bar{\beta}} \nabla_{\alpha} \nabla_{\bar{\beta}} \eta_{\bar{\gamma}}-2 i \nabla_{0} \eta_{\bar{\gamma}}+\eta_{\bar{\rho}} R_{\bar{\gamma}}^{\bar{\rho}}\right) \theta^{\bar{\gamma}},
$$

for any $\eta=\eta_{\bar{\gamma}} \theta^{\bar{\gamma}} \in \Omega^{1,0}(U)$. Consequently

$$
\begin{gathered}
\bar{\square}_{b}\left(\pi_{1,0} \varphi_{j}^{i}\right)=\left(\square_{b}\left(\pi_{1,0} \varphi_{j}^{i}\right)^{-}\right)^{-}= \\
=\left(-g^{\alpha \bar{\beta}} \nabla_{\bar{\beta}} \nabla_{\alpha} \varphi_{j \lambda}^{i}+2 i \nabla_{0} \varphi_{j \lambda}^{i}+\varphi_{j}^{i}\left(T_{\rho}\right) R_{\lambda}^{\rho}\right) \theta^{\lambda}
\end{gathered}
$$

hence

$$
\begin{gathered}
\left(\Delta_{b}^{D} \varphi\right)\left(T_{\lambda}\right) \theta^{\lambda} \otimes e_{j}=2 \bar{\square}_{b}\left(\pi_{1,0} \varphi_{j}^{i}\right) e_{i}+\left\{2(n-1) \sqrt{-1} \nabla_{0} \varphi_{j \lambda}^{i}-\right. \\
\left.-\varphi_{j}^{i}\left(T_{\bar{\rho}}\right) g^{\alpha \bar{\beta}} R_{\bar{\beta}_{\alpha \lambda}{ }^{\rho}}-\varphi_{j}^{i}\left(T_{\rho}\right)\left[2{R_{\lambda}^{\rho}}_{\lambda}+g^{\alpha \bar{\beta}}\left(R_{\alpha}{ }^{\rho} \bar{\beta}_{\lambda}+R_{\lambda}{ }^{\rho} \bar{\beta}_{\alpha}\right)\right]\right\} \theta^{\lambda} \otimes e_{i}+\text { l.o.t. }
\end{gathered}
$$


To compute the curvature terms we need the identities

$$
\begin{gathered}
R_{\lambda \bar{\mu}}=R_{\lambda}{ }^{\alpha}{ }_{\alpha \bar{\mu}}, \\
R_{\alpha}{ }^{\rho}{ }_{\lambda \mu}=2 i\left(A_{\mu \alpha} \delta_{\lambda}^{\rho}-A_{\lambda \alpha} \delta_{\mu}^{\rho}\right), \\
R_{\alpha}{ }^{\rho}{ }_{\bar{\lambda} \bar{\mu}}=2 i\left(g_{\alpha \bar{\lambda}} A_{\bar{\mu}}^{\rho}-g_{\alpha \bar{\mu}} A_{\bar{\lambda}}^{\rho}\right), \\
R_{\alpha \bar{\beta} \lambda \bar{\mu}}=R_{\lambda \bar{\beta} \alpha \bar{\mu}}, \quad R_{\alpha \bar{\beta} \lambda \bar{\mu}}=-R_{\bar{\beta} \alpha \lambda \bar{\mu}},
\end{gathered}
$$

following essentially by the techniques developed in 34. Indeed we have

$$
\begin{gathered}
g^{\alpha \bar{\beta}} R_{\bar{\beta}^{\bar{\rho}}}{ }_{\alpha \lambda}=-2 i(n-1) A_{\lambda}^{\bar{\rho}}, \\
2 R_{\lambda}^{\rho}+g^{\alpha \bar{\beta}}\left(R_{\alpha}{ }^{\rho} \bar{\beta}_{\lambda}+R_{\lambda}{ }^{\rho} \bar{\beta}_{\alpha}\right)=0,
\end{gathered}
$$

hence

$$
\begin{gathered}
\left(\Delta_{b}^{D} \varphi\right)\left(T_{\lambda}\right) \theta^{\lambda} \otimes e_{j}=2 \bar{\square}_{b}\left(\pi_{1,0} \varphi_{j}^{i}\right) \otimes e_{i}+ \\
+2(n-1) \sqrt{-1}\left[\left(\nabla_{T} \varphi_{j}^{i}\right) T_{\lambda}+A_{\lambda}^{\bar{\rho}} \varphi_{j}^{i}\left(T_{\bar{\rho}}\right)\right] \theta^{\lambda} \otimes e_{i}+\text { l.o.t. }
\end{gathered}
$$

Recall that $\tau T_{\alpha}=A_{\alpha}^{\bar{\beta}} T_{\bar{\beta}}$. Then (99) together with the similar identity $\left(\Delta_{b}^{D} \varphi\right)\left(T_{\bar{\lambda}}\right) e_{j}=2\left\{\left(\square_{b} \pi_{0,1} \varphi_{j}^{i}\right)_{\bar{\lambda}}-(n-1) \sqrt{-1}\left(\nabla_{0} \varphi_{j \bar{\lambda}}^{i}+A_{\bar{\lambda}}^{\rho} \varphi_{j \rho}^{i}\right)\right\} e_{i}+$ l.o.t. leads to

$$
\begin{aligned}
& \left(\Delta_{b}^{D} \varphi\right) \otimes e_{j}=2\left\{\square_{b}\left(\pi_{0,1} \varphi_{j}^{i}\right)+\square_{b}\left(\pi_{1,0} \varphi_{j}^{i}\right)+\right. \\
& \left.+(n-1)\left(\nabla_{T} \varphi_{j}^{i}+\varphi_{j}^{i} \circ \tau\right) \circ J\right\} \otimes e_{i}+\text { l.o.t. }
\end{aligned}
$$

and therefore to (13) when $\varphi$ is a $(0,1)$-form. Finally, let us show that $\square_{b}$ is subelliptic on (scalar) $(0,1)$-forms. As $\nabla \omega=0$, the sublaplacian may be computed as

$$
\Delta_{b} f=-\operatorname{trace}\left\{T_{A} \mapsto \nabla_{T_{A}} \nabla^{H} f\right\}=-\nabla_{\alpha} f^{\alpha}-\nabla_{\bar{\alpha}} f^{\bar{\alpha}},
$$

for any $C^{\infty}$ function $f: M \rightarrow \mathbb{C}$, where $f^{\alpha}=g^{\alpha \bar{\beta}} T_{\bar{\beta}}(f)$. As $\nabla g_{\theta}=0$

$$
g^{\alpha \bar{\beta}} \nabla_{\alpha} \nabla_{\bar{\beta}} \eta_{\bar{\lambda}}=\nabla_{\alpha}\left(g^{\alpha \bar{\beta}} \nabla_{\bar{\beta}} \eta_{\bar{\lambda}}\right)=\nabla_{\alpha}\left(\eta_{\bar{\lambda}}\right)^{\alpha}-\nabla_{\alpha}\left(g^{\alpha \bar{\beta}} \Gamma_{\bar{\beta}}^{\bar{\rho}} \eta_{\bar{\lambda}}\right) .
$$

A similar expression holds for $g^{\alpha \bar{\beta}}\left(\nabla_{\bar{\beta}} \nabla_{\alpha} \eta_{\bar{\lambda}}\right)$. Adding up the two identities leads to

$$
g^{\alpha \bar{\beta}}\left(\nabla_{\alpha} \nabla_{\bar{\beta}} \eta_{\bar{\lambda}}+\nabla_{\bar{\beta}} \nabla_{\alpha} \eta_{\bar{\lambda}}\right)=-\Delta_{b} \eta_{\bar{\lambda}}+\text { l.o.t. }
$$

or (by the commutation formulae for the second order derivatives and (98) )

$$
\begin{aligned}
& 2\left(\square_{b} \eta\right)_{\bar{\lambda}}-2 i(n-1) \nabla_{0} \eta_{\bar{\lambda}}-\eta_{\bar{\rho}} R_{\bar{\lambda}}^{\bar{\rho}}= \\
= & \Delta_{b} \eta_{\bar{\lambda}}+g^{\alpha \bar{\beta}}\left\{\nabla_{\alpha}\left(\Gamma_{\bar{\beta}}^{\bar{\rho}} \eta_{\bar{\rho}}\right)+\nabla_{\bar{\beta}}\left(\Gamma_{\alpha \bar{\lambda}}^{\bar{\rho}} \eta_{\bar{\rho}}\right)\right\} .
\end{aligned}
$$

Hence $\square_{b}$ is subelliptic on $\Omega^{0,1}(M)$, i.e. $\square_{b} \eta$ is locally given by a subelliptic operator acting on the coefficients of $\eta$, plus lower order terms. In particular (by (13) $)\left(\Delta_{b}^{D} \varphi\right) \otimes e_{j}=\Delta_{b}\left(\varphi_{j}^{i}\left(T_{\bar{\alpha}}\right)\right) \theta^{\bar{\alpha}} \otimes e_{i}+$ l.o.t. Theorem 3 is completely proved. 


\section{Appendix A. The Graham-Lee Connection}

Let $\Omega=\{\varphi<0\} \subset \mathbb{C}^{n}$ be a strictly pseudoconvex domain and $\mathcal{F}$ the foliation by level sets of $\varphi$ of a one-sided neighborhood $V$ of $\partial \Omega$ (as in section 3 of this paper). Let $\left\{W_{\alpha}: 1 \leq \alpha \leq n-1\right\}$ be a local frame of $T_{1,0}(\mathcal{F})$, so that $\left\{W_{\alpha}, \xi\right\}$ is a local frame of $T^{1,0}(V)$. Let $g_{\theta}$ be the tensor field given by

$$
g_{\theta}(X, Y)=(d \theta)(X, J Y), \quad g_{\theta}(X, T)=0, \quad g_{\theta}(T, T)=1,
$$

for any $X, Y \in H(\mathcal{F})$. Then $g_{\theta}$ is a tangential Riemannian metric for $\mathcal{F}$, i.e. a Riemannian metric in $T(\mathcal{F}) \rightarrow V$. We consider as well

$$
L_{\theta}(Z, \bar{W}) \equiv-i(d \theta)(Z, \bar{W}), \quad Z, W \in T_{1,0}(\mathcal{F}) .
$$

Note that $L_{\theta}$ and (the $\mathbb{C}$-linear extension of) $g_{\theta}$ coincide on $T_{1,0}(\mathcal{F}) \otimes$ $T_{0,1}(\mathcal{F})$. We set $g_{\alpha \bar{\beta}}=g_{\theta}\left(W_{\alpha}, W_{\bar{\beta}}\right)$. Let $\left\{\theta^{\alpha}: 1 \leq \alpha \leq n-1\right\}$ be the (locally defined) complex 1 -forms on $V$ determined by

$$
\theta^{\alpha}\left(W_{\beta}\right)=\delta_{\beta}^{\alpha}, \quad \theta^{\alpha}\left(W_{\bar{\beta}}\right)=0, \quad \theta^{\alpha}(T)=0, \quad \theta^{\alpha}(N)=0 .
$$

Then $\left\{\theta^{\alpha}, \theta^{\bar{\alpha}}, \theta, d \varphi\right\}$ is a local frame of $T(V) \otimes \mathbb{C}$ and we may look for $d \theta$ in the form

$$
\begin{gathered}
d \theta=B_{\alpha \beta} \theta^{\alpha} \wedge \theta^{\beta}+B_{\alpha \bar{\beta}} \theta^{\alpha} \wedge \theta^{\bar{\beta}}+B_{\bar{\alpha} \bar{\beta}} \theta^{\bar{\alpha}} \wedge \theta^{\bar{\beta}}+ \\
+\left(B_{\alpha} \theta^{\alpha}+B_{\bar{\alpha}} \theta^{\bar{\alpha}}\right) \wedge \theta+\left(C_{\alpha} \theta^{\alpha}+C_{\bar{\alpha}} \theta^{\bar{\alpha}}\right) \wedge d \varphi+D d \varphi \wedge \theta .
\end{gathered}
$$

As $d \theta=i \partial \bar{\partial} \varphi \in \Omega^{1,1}(U)$ it follows that $B_{\alpha \beta}=0, B_{\bar{\alpha} \bar{\beta}}=0$. Also

$$
g_{\alpha \bar{\beta}}=g_{\theta}\left(W_{\alpha}, W_{\bar{\beta}}\right)=-i(d \theta)\left(W_{\alpha}, W_{\bar{\beta}}\right)=-\frac{i}{2} B_{\alpha \bar{\beta}}
$$

i.e. $B_{\alpha \bar{\beta}}=2 i g_{\alpha \bar{\beta}}$. Next

$$
\frac{1}{2} B_{\alpha}=(d \theta)\left(W_{\alpha}, T\right)=i \partial \bar{\partial} \varphi\left(W_{\alpha}, T\right)=0
$$

as $T=i(\xi-\bar{\xi})$ (and $\xi$ is orthogonal to $T_{1,0}(\mathcal{F})$ with respect to $\partial \bar{\partial} \varphi$ ), i.e. $B_{\alpha}=0, B_{\bar{\alpha}}=0$. Similarly $C_{\alpha}=0, C_{\bar{\alpha}}=0$. Finally

$$
D=(d \theta)(N, T)=i \partial \bar{\partial} \varphi(N, T)=2 \partial \bar{\partial} \varphi(\xi, \bar{\xi})=r
$$

i.e. $D=r$. We obtain the identity

$$
d \theta=2 i g_{\alpha \bar{\beta}} \theta^{\alpha} \wedge \theta^{\bar{\beta}}+r d \varphi \wedge \theta .
$$

As an immediate consequence

$$
i_{T} d \theta=-\frac{r}{2} d \varphi,
$$

$$
i_{N} d \theta=r \theta .
$$


For instance (by (101)

$$
(d \theta)(X, T)=\frac{r}{2}\{(d \varphi)(X)-(d \varphi)(T) \theta(X)\},
$$

for any $X \in T(\mathcal{F})$, hence (as $(d \varphi)(T)=0$ ) one derives (102). As an application of (101) we decompose $[T, N]$ (according to $T(V) \otimes \mathbb{C}=$ $\left.T_{1,0}(\mathcal{F}) \oplus T_{0,1}(\mathcal{F}) \oplus \mathbb{C} T \oplus \mathbb{C} N\right)$. This is a bit trickier, as shown below. By (102)

$$
\theta([T, N])=-2(d \theta)(T, N)=r d \varphi(N)=2 r .
$$

Next

$$
\begin{gathered}
2(d \theta)\left(W_{\alpha},[T, N]\right)=2 W_{\alpha}(r)-\theta\left(\left[W_{\alpha},[T, N]\right]\right)=\left(J_{a c o b i}^{\prime} s \text { identity }\right) \\
=2 W_{\alpha}(r)+\theta\left(\left[T,\left[N, W_{\alpha}\right]\right]\right)+\theta\left(\left[N,\left[W_{\alpha}, T\right]\right]\right)= \\
=2 W_{\alpha}(r)+2(d \theta)\left(T,\left[W_{\alpha}, N\right]\right)-T\left(\theta\left(\left[W_{\alpha}, N\right]\right)\right)+ \\
+2(d \theta)\left(N,\left[T, W_{\alpha}\right]\right)-N\left(\theta\left(\left[T, W_{\alpha}\right]\right)\right)
\end{gathered}
$$

hence (by (102)-(103)

$$
(d \theta)\left(W_{\alpha},[T, N]\right)=W_{\alpha}(r) .
$$

We conclude that

$$
[T, N]=i W^{\alpha}(r) W_{\alpha}-i W^{\bar{\alpha}}(r) W_{\bar{\alpha}}+2 r T,
$$

where $W^{\alpha}(r)=g^{\alpha \bar{\beta}} W_{\bar{\beta}}(r)$ and $W^{\bar{\alpha}}(r)=\overline{W^{\alpha}(r)}$.

Let $\nabla$ be a linear connection on $V$. Let us consider the $T(V)$-valued 1-form $\tau$ on $V$ defined by

$$
\tau(X)=T_{\nabla}(T, X), \quad X \in T(V),
$$

where $T_{\nabla}$ is the torsion tensor field of $\nabla$. We say $T_{\nabla}$ is pure if

$$
\begin{gathered}
T_{\nabla}(Z, W)=0, \quad T_{\nabla}(Z, \bar{W})=2 i L_{\theta}(Z, \bar{W}) T, \\
T_{\nabla}(N, W)=r W+i \tau(W),
\end{gathered}
$$

for any $Z, W \in T_{1,0}(\mathcal{F})$, and

$$
\begin{gathered}
\tau\left(T_{1,0}(\mathcal{F})\right) \subseteq T_{0,1}(\mathcal{F}), \\
\tau(N)=-J \nabla^{H} r-2 r T .
\end{gathered}
$$

Here $\nabla^{H} r$ is defined by $\nabla^{H} r=\pi_{H} \nabla r$ and $g_{\theta}(\nabla r, X)=X(r), X \in$ $T(\mathcal{F})$. Also $\pi_{H}: T(\mathcal{F}) \rightarrow H(\mathcal{F})$ is the projection associated to the direct sum decomposition $T(\mathcal{F})=H(\mathcal{F}) \oplus \mathbb{R} T$. Appendix $\mathrm{A}$ is aimed at the following 
Theorem 4. (C.R. Graham \& J.M. Lee, [1])

There is a unique linear connection $\nabla$ on $V$ such that i) $T_{1,0}(\mathcal{F})$ is parallel with respect to $\nabla$, ii) $\nabla L_{\theta}=0, \nabla T=0, \nabla N=0$, and iii) $T_{\nabla}$ is pure.

$\nabla$ given by Theorem 4 is the Graham-Lee connection. Compare to Proposition 1.1 in 11, p. 701-702. The axiomatic description in Theorem 4 is however new (cf. also Theorem 2 in [7]). We first establish

Lemma 3. Let $\phi: T(\mathcal{F}) \rightarrow T(\mathcal{F})$ be the bundle morphism given by $\phi(X)=J X$, for any $X \in H(\mathcal{F})$, and $\phi(T)=0$. Then

$$
\begin{gathered}
\phi^{2}=-I+\theta \otimes T, \\
g_{\theta}(X, T)=\theta(X), \\
g_{\theta}(\phi X, \phi Y)=g_{\theta}(X, Y)-\theta(X) \theta(Y),
\end{gathered}
$$

for any $X, Y \in T(\mathcal{F})$. Moreover, if $\nabla$ is a linear connection on $V$ satisfying the axioms (i)-(iii) in Theorem 4 then

$$
\phi \circ \tau+\tau \circ \phi=0
$$

along $T(\mathcal{F})$. Consequently $\tau$ may be computed as

$$
\tau(X)=-\frac{1}{2} \phi\left(\mathcal{L}_{T} \phi\right) X
$$

for any $X \in H(\mathcal{F})$.

Proof. For any $X \in T(\mathcal{F})$

$$
\begin{gathered}
\phi(X)=\phi\left(\pi_{H} X+\theta(X) T\right)=J\left(\pi_{H} X\right) \in H(\mathcal{F}), \\
\phi^{2}(X)=J^{2}\left(\pi_{H} X\right)=-\pi_{H} X=-X+\theta(X) T .
\end{gathered}
$$

The second statement in Lemma 3 follows from definitions (cf. (100)). The third identity follows from

$$
\begin{gathered}
g_{\theta}(\phi X, \phi Y)=(d \theta)\left(\phi \pi_{H} X, \phi^{2} \pi_{H} Y\right)= \\
=g_{\theta}\left(\pi_{H} Y, \pi_{H} X\right)=g_{\theta}(Y, X)-\theta(X) g_{\theta}(Y, T) .
\end{gathered}
$$

Let us prove (109). As $\tau\left(T_{1,0}(\mathcal{F})\right) \subseteq T_{0,1}(\mathcal{F})$ (cf. axiom (107)) there are complex valued functions $A_{\alpha}^{\bar{\beta}}$ such that $\tau\left(W_{\alpha}\right)=A_{\alpha}^{\bar{\beta}} W_{\bar{\beta}}$. Then

$$
(\tau \circ \phi+\phi \circ \tau) W_{\alpha}=i \tau\left(W_{\alpha}\right)+A_{\alpha}^{\bar{\beta}} \phi\left(W_{\bar{\beta}}\right)=0 .
$$

It remains that we check (110). As $T_{1,0}(\mathcal{F})$ is parallel with respect to $\nabla$ and $\nabla$ is a real operator it follows that $T_{0,1}(\mathcal{F})$ is parallel, hence both $H(\mathcal{F})$ and its complex structure $\left.J_{\mathcal{F}} \equiv J\right|_{H(\mathcal{F})}$ are parallel. Moreover, as $\nabla T=0$, it follows that $\phi$ is parallel, as well. Let $X \in H(\mathcal{F})$. Then (by (109) )

$$
\phi \tau X=-T_{\nabla}(T, \phi X)=-\nabla_{T} \phi X+[T, \phi X] .
$$


Applying $\phi$ in both sides gives (as $\nabla \phi=0$ )

$$
\tau X=-\nabla_{T} X-\phi[T, \phi X]=-[T, X]-\tau X-\phi[T, \phi X]
$$

or

$$
2 \tau X=-\mathcal{L}_{T} X-\phi \mathcal{L}_{T} \phi X
$$

Q.e.d.

Proof of Theorem 4. To establish uniqueness, note first that, for any $X=X^{1,0}+X^{0,1}+\theta(X) T \in T(\mathcal{F})\left(\right.$ with $\left.X^{1,0} \in T_{1,0}(\mathcal{F}), X^{0,1}=\overline{X^{1,0}}\right)$ one has (by $\nabla N=0$ )

$$
\begin{gathered}
\nabla_{N} X=[N, X]+T_{\nabla}(N, X)=(b y(105)-(106),(108)) \\
=[N, X]+r X^{1,0}+i \tau X^{1,0}+r X^{0,1}-i \tau X^{0,1}+\theta(X)\left\{J \nabla^{H} r+2 r T\right\}
\end{gathered}
$$

that is

$$
\nabla_{N} X=r X+\tau \phi X-[X, N]+\theta(X)\left\{J \nabla^{H} r+r T\right\},
$$

for any $X \in T(\mathcal{F})$. In view of (110) $\nabla_{N} X$ is determined. As $\nabla N=0$, $\nabla T=0$ it remains that we compute $\nabla_{X} Z$, for $X \in T(\mathcal{F})$ and $Z \in$ $T_{1,0}(\mathcal{F})$. Note that $\nabla T=0, \nabla L_{\theta}=0$ and $\nabla J_{\mathcal{F}}=0$ yield $\nabla g_{\theta}=0$, i.e.

$$
X\left(g_{\theta}(Y, Z)\right)=g_{\theta}\left(\nabla_{X} Y, Z\right)+g_{\theta}\left(Y, \nabla_{X} Z\right),
$$

for any $X, Y, Z \in T(\mathcal{F})$. The well known Christoffel process then leads to

$$
\begin{gathered}
2 g_{\theta}\left(\nabla_{X} Y, Z\right)=X\left(g_{\theta}(Y, Z)\right)+Y\left(g_{\theta}(X, Z)\right)-Z\left(g_{\theta}(X, Y)\right)+ \\
+g_{\theta}([X, Y], Z)+g_{\theta}\left(T_{\nabla}(X, Y), Z\right)+ \\
+g_{\theta}([Z, X], Y)+g_{\theta}\left(T_{\nabla}(Z, X), Y\right)+ \\
+g_{\theta}(X,[Z, Y])+g_{\theta}\left(X, T_{\nabla}(Z, Y)\right)
\end{gathered}
$$

Note that (again by the purity axioms)

$$
T_{\nabla}(X, Y)=2(d \theta)(X, Y) T+2(\theta \wedge \tau)(X, Y),
$$

for any $X, Y \in T(\mathcal{F})$. Indeed (by (105))

$$
T_{\nabla}(X, Y)=-2 g_{\theta}(X, \phi Y) T+2(\theta \wedge \tau)(X, Y) .
$$

Moreover

$$
\begin{gathered}
g_{\theta}(X, \phi Y)=g_{\theta}\left(\pi_{H} X, \phi \pi_{H} Y\right)+\theta(X) g_{\theta}\left(T, \phi \pi_{H} Y\right)= \\
=-(d \theta)\left(\pi_{H} X, \pi_{H} Y\right)=-(d \theta)(X, Y)+ \\
+\theta(X)(d \theta)(T, Y)+\theta(Y)(d \theta)(X, T) .
\end{gathered}
$$

Finally (by (102)) $(d \theta)(X, T)=0, X \in T(\mathcal{F})$, and (113) is proved. Replacing the torsion terms (from (113) into (112)) leads to

$$
\begin{aligned}
& 2 g_{\theta}\left(\nabla_{X} Z, \bar{W}\right)=X\left(g_{\theta}(Z, \bar{W})\right)+Z\left(g_{\theta}(X, \bar{W})\right)-\bar{W}\left(g_{\theta}(X, Z)\right)+ \\
& +g_{\theta}([X, Z], \bar{W})+g_{\theta}([\bar{W}, X], Z)+g_{\theta}(X,[\bar{W}, Z])
\end{aligned}
$$


for any $X \in T(\mathcal{F})$ and $Z, W \in T_{1,0}(\mathcal{F})$, as (by (107))

$$
\theta(X)\left\{g_{\theta}(\tau Z, \bar{W})-g_{\theta}(\tau \bar{W}, Z)\right\}=0 .
$$

The uniqueness statement in Theorem 4 is proved. The following explicit expressions of (the various components of) $\nabla$ are also available. By (105)

$$
\nabla_{Z} \bar{W}=\pi_{0,1}[Z, \bar{W}], \quad Z, W \in T_{1,0}(\mathcal{F}),
$$

where $\pi_{0,1}: T(\mathcal{F}) \otimes \mathbb{C} \rightarrow T_{0,1}(\mathcal{F})$ is the projection. Of course

$$
\nabla_{\bar{Z}} W=\overline{\nabla_{Z} \bar{W}}
$$

Moreover (by $\nabla L_{\theta}=0$ and (115)

$$
L_{\theta}\left(\nabla_{Z} W, \bar{V}\right)=Z\left(L_{\theta}(W, \bar{V})\right)-L_{\theta}\left(W, \pi_{0,1}[Z, \bar{V}]\right),
$$

for any $Z, W, V \in T_{1,0}(\mathcal{F})$, i.e.

$$
\nabla_{Z} W=g^{\alpha \bar{\beta}}\left\{Z\left(L_{\theta}\left(W, W_{\bar{\beta}}\right)\right)-L_{\theta}\left(W, \pi_{0,1}\left[Z, W_{\bar{\beta}}\right]\right)\right\} W_{\alpha}
$$

and

$$
\nabla_{\bar{Z}} \bar{W}=\overline{\nabla_{Z} W}
$$

Next (by (106)-(107))

$$
\nabla_{N} Z=r Z+\pi_{1,0}[N, Z]
$$

for any $Z \in T_{1,0}(\mathcal{F})$, and

$$
\nabla_{N} \bar{Z}=\overline{\nabla_{N} Z}
$$

Finally

$$
\begin{gathered}
\nabla_{T} Z=-\frac{1}{2} \phi\left(\mathcal{L}_{T} \phi\right) Z-[Z, T], \\
\nabla_{T} \bar{Z}=\overline{\nabla_{T} Z}, \quad Z \in T_{1,0}(\mathcal{F}) .
\end{gathered}
$$

To establish the existence statement in Theorem 4 let $\nabla$ be the linear connection on $V$ defined by (115)-(118) and $\nabla T=0, \nabla N=0$. Let us check (i)-(iii) in Theorem 4. Clearly

$$
\nabla_{Z} W, \quad \nabla_{\bar{Z}} W, \quad \nabla_{N} W \in T_{1,0}(\mathcal{F}),
$$

for any $W \in T_{1,0}(\mathcal{F})$, by the very definitions (cf. (115)-(117)). Moreover (by (118) )

$$
\nabla_{T} Z=\frac{1}{2}\left(\mathcal{L}_{T} Z-i \phi \mathcal{L}_{T} Z\right)
$$

as $($ by $(102)) \mathcal{L}_{T} Z \in H(\mathcal{F}) \otimes \mathbb{C}$. Therefore

$$
\phi \nabla_{T} Z=i \nabla_{T} Z
$$


that is as $T_{1,0}(\mathcal{F})$ is the eigenspace corresponding to the eigenvalue $i$ of (the $\mathbb{C}$-linear extension to $H(\mathcal{F})$ of $) \phi] \nabla_{T} Z \in T_{1,0}(\mathcal{F})$. We conclude that $\nabla$ obeys to (i). Let us check purity. By (116)

$$
\begin{gathered}
L_{\theta}\left(\nabla_{Z} W-\nabla_{W} Z-[Z, W], \bar{V}\right)= \\
=Z\left(L_{\theta}(W, \bar{V})\right)-L_{\theta}\left(W, \pi_{0,1}[Z, \bar{V}]\right)- \\
-W\left(L_{\theta}(Z, \bar{V})\right)+L_{\theta}\left(Z, \pi_{0,1}[W, \bar{V}]\right)-L_{\theta}([Z, W], \bar{V})= \\
=3\left(d^{2} \theta\right)(Z, W, \bar{V})=0 .
\end{gathered}
$$

Therefore $T_{\nabla}(Z, W)=0$. Next (by (115) and $\overline{\pi_{0,1} X}=\pi_{1,0} \bar{X}, X \in$ $T(\mathcal{F}) \otimes \mathbb{C})$

$$
\begin{gathered}
T_{\nabla}(Z, \bar{W})=\pi_{0,1}[Z, \bar{W}]-\overline{\pi_{0,1}[W, \bar{Z}]}-[Z, \bar{W}]= \\
=-\theta([Z, \bar{W}]) T=2 i L_{\theta}(Z, \bar{W}) T .
\end{gathered}
$$

Moreover (by (117))

$$
T_{\nabla}(N, Z)=\nabla_{N} Z-[N, Z]=r Z-\pi_{0,1}[N, Z] .
$$

Also (by (118))

$$
\tau(Z)=-\frac{1}{2} \phi\left(\mathcal{L}_{T} \phi\right) Z, \quad Z \in T_{1,0}(\mathcal{F}),
$$

so that on one hand (107) is satisfied, and on the other

$$
\begin{gathered}
\tau(Z)=-\frac{1}{2}\{i \phi[T, Z]+[T, Z]\}=-\pi_{0,1}[T, Z]= \\
=i\left\{\pi_{0,1}[\bar{\xi}, Z]-\pi_{0,1}[\xi, Z]\right\}=i \pi_{0,1}[\bar{\xi}, Z]
\end{gathered}
$$

i.e.

$$
\tau(Z)=i \pi_{0,1}[N, Z], \quad Z \in T_{1,0}(\mathcal{F}) .
$$

Here we made use of $T=i(\xi-\bar{\xi}), N=\xi+\bar{\xi}$ and $\pi_{0,1}[\xi, Z]=0$. Then (119) yields (106). Finally $\nabla T=\nabla N=0$ and (104) yield (108) and we conclude that $\nabla$ obeys to (iii). It remains that we check $\nabla L_{\theta}=0$. Clearly $\nabla_{Z} L_{\theta}=0, Z \in T_{1,0}(\mathcal{F})$ (by (115)-(116)). Next (by (118) and (107) )

$$
\left(\nabla_{T} L_{\theta}\right)(Z, \bar{W})=\left(\mathcal{L}_{T} L_{\theta}\right)(Z, \bar{W}), \quad Z, W \in T_{1,0}(\mathcal{F})
$$

and

$$
\begin{gathered}
\left(\mathcal{L}_{T} L_{\theta}\right)(Z, \bar{W})=-i\{T(d \theta(Z, \bar{W}))-d \theta([T, Z], \bar{W})-d \theta(Z,[T, \bar{W}])\}= \\
=\frac{i}{2}\{T(\theta([Z, \bar{W}]))-\theta([[T, Z], \bar{W}])-\theta([Z,[T, \bar{W}]])\}=
\end{gathered}
$$

(by applying the Jacobi identity to the term $\theta([[T, Z], \bar{W}]))$

$$
=\frac{i}{2}\{T(\theta([Z, \bar{W}]))+\theta([[Z, \bar{W}], T])\}=
$$




$$
=i(d \theta)(T,[Z, \bar{W}])=-\frac{i r}{2}(d \varphi)([Z, \bar{W}])=0
$$

(by (102) and $[Z, \bar{W}] \in T(\mathcal{F}) \otimes \mathbb{C}$ ). Hence $\nabla_{T} L_{\theta}=0$. Finally (by (117))

$$
\left(\nabla_{N} L_{\theta}\right)(Z, \bar{W})=-2 r g_{\theta}(Z, \bar{W})+\left(\mathcal{L}_{N} g_{\theta}\right)(Z, \bar{W}), \quad Z, W \in T_{1,0}(\mathcal{F}),
$$

and $\nabla_{N} L_{\theta}=0$ follows from (122) in Lemma 4 below.

Lemma 4. The following identities hold for any $X \in T(\mathcal{F})$

$$
\begin{gathered}
T_{\nabla}(N, X)=r X+\tau(\phi X)+\theta(X)\left\{\phi \nabla^{H} r+r T\right\}, \\
{[N, \phi X]-\phi[N, X]=2 \tau(X)-\theta(X) \nabla^{H} r,}
\end{gathered}
$$

Moreover

$$
\left(\mathcal{L}_{N} g_{\theta}\right)(X, Y)=2 r g_{\theta}(X, Y)+2(d \theta)(X, \tau(Y)),
$$

for any $X, Y \in H(\mathcal{F})$.

Proof. (120) follows from (106). Let us replace $X$ by $\phi X$ in (120)

$$
\nabla_{N} \phi X-[N, \phi X]=r \phi X-\tau(X)
$$

and subtract the identity got from (120) by applying $\phi$ to both sides. Since $\nabla \phi=0$ we obtain (121). The proof of (122) is a consequence of (103), (121), and the Jacobi identity

$$
\begin{gathered}
\left(\mathcal{L}_{N} g_{\theta}\right)(X, Y)=N((d \theta)(X, \phi Y))-(d \theta)([N, X], \phi Y)+ \\
+2(d \theta)(X, \tau(Y))-(d \theta)(X,[N, \phi Y])= \\
=-\frac{1}{2} N(\theta([X, \phi Y]))+\frac{1}{2} \theta([[N, X], \phi Y])+ \\
+2(d \theta)(X, \tau(Y))+\frac{1}{2} \theta([X,[N, \phi Y]])= \\
=-\frac{1}{2} N(\theta([X, \phi Y]))-\frac{1}{2} \theta([[X, \phi Y], N])-\theta([X, \tau(Y)])= \\
=-(d \theta)(N,[X, \phi Y])-\theta([X, \tau(Y)])= \\
=-r \theta([X, \phi Y])-\theta([X, \tau(Y)])= \\
=2 r(d \theta)(X, \phi Y)+2(d \theta)(X, \tau(Y))= \\
=2 r g_{\theta}(X, Y)+2(d \theta)(X, \tau(Y)) .
\end{gathered}
$$

Q.e.d. Theorem 4 is proved.

As to the local calculations, if $\varphi_{\beta}^{\alpha}$ are the connection 1-forms of the Graham-Lee connection (i.e. $\nabla W_{\beta}=\varphi_{\beta}^{\alpha} \otimes W_{\alpha}$ ) then we may look for $d \theta^{\alpha}$ in the form

$$
d \theta^{\alpha}=B_{\beta \gamma}^{\alpha} \theta^{\beta} \wedge \theta^{\gamma}+B_{\beta \bar{\gamma}}^{\alpha} \theta^{\beta} \wedge \theta^{\bar{\gamma}}+B_{\bar{\beta} \bar{\gamma}}^{\alpha} \theta^{\bar{\beta}} \wedge \theta^{\bar{\gamma}}+
$$




$$
+\left(B_{\beta}^{\alpha} \theta^{\beta}+B_{\bar{\beta}}^{\alpha} \theta^{\bar{\beta}}\right) \wedge \theta+\left(C_{\beta}^{\alpha} \theta^{\beta}+C_{\bar{\beta}}^{\alpha} \theta^{\bar{\beta}}\right) \wedge d \varphi+D d \varphi \wedge \theta .
$$

Indeed, applying this identity to the pair $\left(W_{\beta}, W_{\gamma}\right)$ (respectively to $\left(W_{\beta}, W_{\bar{\gamma}}\right)$ and $\left.\left(W_{\beta}, T\right)\right)$ gives

$$
B_{\beta \gamma}^{\alpha}-B_{\gamma \beta}^{\alpha}=\varphi_{\beta}^{\alpha}\left(W_{\gamma}\right)-\varphi_{\gamma}^{\alpha}\left(W_{\beta}\right), \quad B_{\beta \bar{\gamma}}^{\alpha}=\varphi_{\beta}^{\alpha}\left(W_{\bar{\gamma}}\right), \quad B_{\beta}^{\alpha}=\varphi_{\beta}^{\alpha}(T) .
$$

Similarly (applying (123) to $\left(W_{\bar{\beta}}, T\right),\left(W_{\beta}, N\right)$ and $\left(W_{\bar{\beta}}, N\right)$ respectively)

$$
B_{\bar{\beta}}^{\alpha}=-A_{\bar{\beta}}^{\alpha}, \quad C_{\beta}^{\alpha}=\frac{1}{2}\left(\varphi_{\beta}^{\alpha}(N)-r \delta_{\beta}^{\alpha}\right), \quad C_{\bar{\beta}}^{\alpha}=\frac{i}{2} A_{\bar{\beta}}^{\alpha} .
$$

Finally (by (104) $)$

$$
D d \varphi(N)=2\left(d \theta^{\alpha}\right)(N, T)=-\theta^{\alpha}([N, T])=i W^{\alpha}(r) .
$$

Summing up

$$
d \theta^{\alpha}=\theta^{\beta} \wedge \varphi_{\beta}^{\alpha}-i \partial \varphi \wedge \tau^{\alpha}+\frac{i}{2} W^{\alpha}(r) d \varphi \wedge \theta+\frac{r}{2} d \varphi \wedge \theta^{\alpha},
$$

where $\tau^{\alpha} \equiv A \frac{\alpha}{\beta} \theta^{\bar{\beta}}$.

Given a linear connection $\nabla$ on $V$ we set $\alpha(X, Y) \equiv \Pi \nabla_{X} Y$, for any $X, Y \in T(\mathcal{F})$. If $\nabla$ is the Graham-Lee connection then (by the proof of Theorem 4 $\alpha=0$. One may identify, as usual, the normal bundle $\nu(\mathcal{F})=T(V) / T(\mathcal{F})$ with $\mathbb{R} N$. If $\Pi^{\perp}: T(V) \rightarrow T(\mathcal{F})$ is the projection, let us set $\nabla^{\mathcal{F}} \equiv \Pi^{\perp} \nabla$. It is easily seen that $\nabla^{\mathcal{F}}$ is the Tanaka-Webster connection of each $M_{\delta}$ (i.e. the pointwise restriction of the GrahamLee connection to a leaf of $\mathcal{F}$ is the Tanaka-Webster connection of the leaf). In particular $\tau: T(\mathcal{F}) \rightarrow T(\mathcal{F})$ is the pseudohermitian torsion of each leaf (hence $g_{\theta}(\tau X, Y)=g_{\theta}(X, \tau Y)$, for any $X, Y \in T(\mathcal{F})$ ).

\section{REFERENCES}

[1] E. Barletta \& S. Dragomir, On the CR structure of the tangent sphere bundle, Le Matematiche, Catania, (2)L(1995), 237-249.

[2] E. Barletta \& S. Dragomir \& H. Urakawa, Pseudoharmonic maps from a nondegenerate CR manifold into a Riemannian manifold, Indiana University Mathematics Journal, (2)50(2001), 719-746.

[3] J.K. Beem \& P.E. Ehrlich, Global Lorentzian geometry, Marcel Dekker, Inc., New York - Basel, 1981.

[4] J-P. Bourguignon \& H.B. Lawson, Jr., Stability and isolation phenomena for Yang-Mills fields, Commun. Math. Phys., 79(1981), 189-230.

[5] S. Dragomir, Pseudohermitian geometry, Bull. Math. Sc. Math. Roumanie, (3)43(2000), 225-234. 
[6] S. Dragomir \& H. Urakawa, On the inhomogeneous Yang-Mills equation $d_{D}^{*} R^{D}=f$, Interdisciplinary Information Sciences, (1)6(2000), 41-52.

[7] S. Dragomir \& S. Nishikawa, Foliated $C R$ manifolds, J. Math. Soc. Japan, (4)56(2004), 1031-1068.

[8] C. Fefferman, The Bergman kernel and biholomorphic equivalence of pseudoconvex domains, Invent. Math., 26(1974), 1-65.

[9] G.B. Folland \& E.M. Stein, Estimates for the $\bar{\partial}_{b}$-complex and analysis on the Heisenberg group, Comm. Pure Appl. Math., 27(1974), 429-522.

[10] N. Gamara \& R. Yacoub, CR Yamabe conjecture - the conformally flat case, Pacific J. Math., (1)201(2001), 121-175.

[11] C.R. Graham \& J.M. Lee, Smooth solutions of degenerate Laplacians on strictly pseudoconvex domains, Duke Math. J., (3)57(1988), 697-720.

[12] J. Harnad \& S. Shnider \& L. Vinet, Group actions on principal bundles and invariance conditions for gauge fields, J. Math. Phys., 21(1980), 2719.

[13] D. Jerison \& J.M. Lee, The Yamabe problem on CR manifolds, J. Diff. Geometry, 25(1987), 167-197.

[14] D. Jerison \& J.M. Lee, CR normal coordinates and the Yamabe problem, J. Diff. Geometry, 29(1989), 303-344.

[15] J. Jost \& C-J. Xu, Subelliptic harmonic maps, Trans. of A.M.S., (11)350(1998), 4633-4649.

[16] S. Kobayashi \& K. Nomizu, Foundations of differential geometry, Interscience Publishers, New York, vol. I, 1963, vol. II, 1969.

[17] C.R. Lebrun, Twistor CR manifolds and three-dimensional conformal geometry, Trans. A.M.S., (2)284(1984), 601-616.

[18] J.M. Lee, The Fefferman metric and pseudohermitian invariants, Trans. A.M.S., (1)296(1986), 411-429.

[19] J.M. Lee, Pseudo-Einstein structures on CR manifolds, American J. Math., 110(1988), 157-178.

[20] J.M. Lee \& R. Melrose, Boundary behaviour of the complex MongeAmpère equation, Acta Mathematica, 148(1982), 159-192.

[21] J. Lewandowski \& P. Nurowski, Algebrically special twisting gravitational fields and three-dimensional Cauchy-Riemann structures, Classical Quantum Grav., 7(1990), 309.

[22] B. O'Neill, Semi-Riemannian geometry, Academic Press, New YorkLondon-Paris-San Francisco-São Paulo-Sydney-Tokto-Toronto, 1983.

[23] B. O'Neill, The fundamenrtal equations of a submersion, Michigan Math. J., 13(1966), 459-469.

[24] P. Nurowski, Some null solutions of the Yang-Mills equations and Cauchy-Riemann structures, J. Math. Phys., (2)33(1992), 802-807. 
[25] P. Nurowski \& J. Tafel, Symmetries of Cauchy-Riemann spaces, Lett. Math. Phys., 15(1988), 31.

[26] P. Nurowski \& A. Trautman, Robinson manifolds as the Lorentzian analogs of Hermite manifolds, Diff. Geometry Appl., 17(2002), 175195.

[27] E.V. Radkevic, Hypoelliptic operators with multiple characteristics, Math. USSR Sb., 8(1969), 181-205.

[28] J. Tafel, Null solutions of the Yang-Mills equations, Lett. Math. Phys., 12(1986), 163.

[29] N. Tanaka, A differential geometric study on strongly pseudo-convex manifolds, Kinokuniya Book Store Co., Ltd., Kyoto, 1975.

[30] H. Urakawa, Yang-Mills connections over compact strongly pseudoconvex CR manifolds, Math. Z., 216(1994), 541-573.

[31] H. Urakawa, Variational problems over strongly pseudoconvex CR manifolds, Differential Geometry, Proceedings of the Sympos. in honour of Prof. Su Buchin on his 90th birthday, 233-242, Shanghai, China, September 17-23, 1991, Ed. by C.H. Gu \& H.S. Hu \& Y.L. Xin, World Scientific Publ. Co. Pte. Ltd., Singapore-New JerseyLondon-Hong Kong, 1993.

[32] H. Urakawa, Yang-Mills connections and deformation theory over compact strongly pseudoconvex CR manifolds, Proceedings of the Third MSJ International Research Inst. on Geometric Complex Analysis, Hayama, Japan, March 19-29, 1995, World Scientific Publ. Co. Pte. Ltd., 1995.

[33] H. Urakawa, Yang-Mills theory over compact symplectic manifolds, Annals of Global Analysis and Geometry, 25(2004), 365-402.

[34] S.M. Webster, Pseudohermitian structures on a real hypersurface, J. Diff. Geometry, 13(1978), 25-41. 